\title{
The High Luminosity LHC interaction region magnets towards series production
}

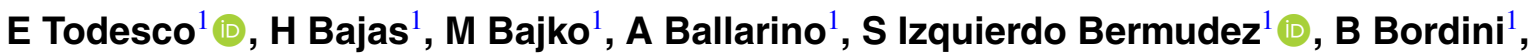 \\ L Bottura ${ }^{1}$, G De Rijk ${ }^{1}$, A Devred ${ }^{1}$, D Duarte Ramos ${ }^{1}$, M Duda ${ }^{1}$, P Ferracin ${ }^{1}$, P Fessia ${ }^{1}$, \\ J Fleiter $^{1}$, L Fiscarelli ${ }^{1}$, A Foussat ${ }^{1}$, G Kirby ${ }^{1}$, F Mangiarotti $^{1}$, M Mentink ${ }^{1}$ (D), A Milanese ${ }^{1}$, \\ A Musso ${ }^{1}$, V Parma ${ }^{1}$, J C Perez ${ }^{1}$, H Prin ${ }^{1}$, L Rossi ${ }^{1}{ }^{1}$, S Russenschuck ${ }^{1}$, G Willering ${ }^{1}$, \\ S Enomoto ${ }^{2}$, T Nakamoto ${ }^{2}$, N Kimura ${ }^{2}$, T Ogitsu ${ }^{2}$, M Sugano $^{2}$ (D, K Suzuki ${ }^{2}, \mathbf{S ~ W e i}^{3}$,

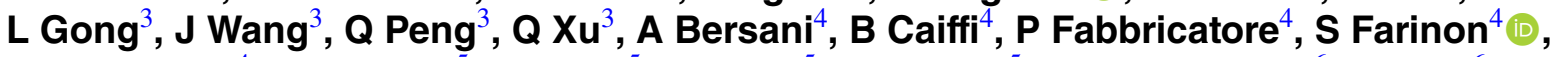

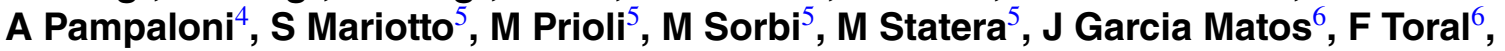 \\ G Ambrosio ${ }^{7}$, G Apollinari ${ }^{7}$, M Baldini $^{7}$, R Carcagno ${ }^{7}$, S Feher ${ }^{7}$, S Stoynev ${ }^{7}$, \\ G Chlachidze $^{7}$, V Marinozzi ${ }^{7}$, V Lombardo ${ }^{7}$, F Nobrega $^{7}$, T Strauss ${ }^{7}$, M Yu $^{7}, \mathbf{M ~ A n e r e l l a ~}^{8}$,

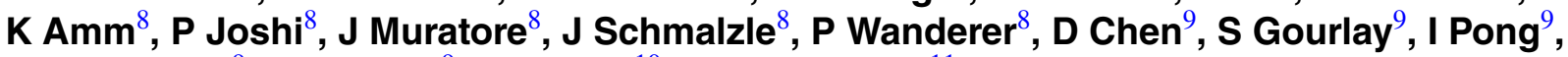 \\ S Prestemon ${ }^{9}$, G L Sabbi $^{9}$, L Cooley ${ }^{10}$ (i) and H Felice ${ }^{11}$ \\ ${ }^{1}$ TE Department, CERN, Geneve, Switzerland \\ ${ }^{2}$ KEK, Tsukuba, Japan \\ ${ }^{3}$ IHEP, Beijing, People's Republic of China \\ ${ }^{4}$ INFN, Sezione di Genova, Italy \\ ${ }^{5}$ INFN, Sezione di Milano, Italy \\ ${ }^{6}$ CIEMAT, Spain \\ ${ }^{7}$ FNAL, Batavia, IL, United States of America \\ ${ }^{8}$ BNL, NY, United States of America \\ ${ }^{9}$ LBNL, CA, United States of America \\ ${ }^{10}$ NHMFL, FL, United States of America \\ ${ }^{11}$ CEA, Saclay, France \\ E-mail: ezio.todesco@cern.ch
}

Received 25 May 2020, revised 25 November 2020 Accepted for publication 13 January 2021

Published 22 March 2021

\section{Abstract}

The High Luminosity Large Hadron Collider (HL-LHC) is the new flagship project of CERN. First endorsed in 2013 and approved in 2016, HL-LHC is an upgrade of the accelerator aiming to increase by a factor of ten the statistics of the LHC collisions at the horizon of 2035-2040. HL-LHC relies on cutting edge technologies: among them, large aperture superconducting magnets will replace the present hardware to allow a smaller beam size in two interaction points (IPs). The project involves the construction of about 150 magnets of six different types: the quadrupole triplet, two main dipoles and three orbit correctors. The triplet, manufactured at CERN and in the USA, will consist of 30 magnets based on $\mathrm{Nb}_{3} \mathrm{Sn}$ technology, with an 
operational peak field of $11.4 \mathrm{~T}$. These will be the first quadrupole $\mathrm{Nb}_{3} \mathrm{Sn}$ magnets installed in a particle accelerator. The other five types of magnets, all relying on $\mathrm{Nb}-\mathrm{Ti}$ technology, present non-trivial challenges in the design and construction; they will be manufactured as part of in-kind contribution under the responsibility of institutes in Japan, China, Spain, and Italy. The project is now in the phase of transition between qualification through short models and prototypes and the beginning of the series construction. In this paper we review the magnet requirements, the reasons for selecting the design, the technological challenges with respect to previous projects, and we summarize the steps that have been taken to validate the baseline.

Keywords: superconducting accelerator magnets, $\mathrm{Nb}_{3} \mathrm{Sn}, \mathrm{Nb}-\mathrm{Ti}$

(Some figures may appear in colour only in the online journal)

\section{Introduction}

The Large Hadron Collider (LHC) is the particle collider with the highest energy ever built, designed to collide protons at $7+7 \mathrm{TeV}$ energy [1]. The accelerator relies on superconducting $\mathrm{Nb}-\mathrm{Ti}$ dipoles magnets operating at $\sim 8 \mathrm{~T}$ and $1.9 \mathrm{~K}$ [2], pushing this technology to its limits for a large scale production of accelerator magnets [3]. First proposed in the 1980s [4], the LHC went through a design and prototyping phase in the 1990s [5-7], a construction and installation phase in 2000-2007 [3] and was finally commissioned in 2008-2010 [8]. It led to the discovery of the Higgs boson in 2012 [9, 10], and to the award of a Nobel Prize in 2013 to F. Englert and P. Higgs.

One year before the Higgs boson discovery, the High Luminosity LHC (HL-LHC) design study was launched [11], aiming at a substantial upgrade of the LHC to increase the statistics of the collision data by a factor of ten. This design study was the last stage of a 10 years long period of investigations on the possibilities of improving the LHC performances, started in 2000 [12-14].

The HL-LHC proposal $[15,16]$ is based on a 20 -fold potential increase of the collision rates (peak luminosity), given by a twice larger proton beam intensity (more fuel to burn) and a two times smaller beam size (fuel burnt more rapidly). Since the beam size in the IP is inversly proportional to the aperture of the first magnets after the experiments, a smaller beam size in the IP requires larger aperture magnets [12-16]. Therefore the HL-LHC project requires the replacement of the interaction region (IR) magnets with larger aperture magnets. The selected aperture of the IR magnets in the HL-LHC is $150 \mathrm{~mm}$ [17], i.e. more than twice the $70 \mathrm{~mm}$-aperture of the LHC IR magnets [18-20]. This larger aperture is used not only for reducing the beam size in the IP, but also for housing inside the magnet a $10 \mathrm{~mm}$-thick tungsten beam screen [15, 16]. This shield allows keeping the peak heat loads and the radiation dose induced by collision debris at the same level of the LHC (respectively, $5 \mathrm{~mW} \mathrm{~cm}^{-3}$ and $35 \mathrm{MGy}$ over the expected LHC lifetime).

An essential ingredient of the IR magnets upgrade is the triplet, that is the sequence of the first three quadrupole magnets in front of the experiments, needed to match the beam parameters from the highly focused conditions at the IP to the beam envelope requested in the curved sections of the accelerator (arcs). To guarantee the required gradient in such a large aperture, a change of technology is required, i.e. switching from $\mathrm{Nb}-\mathrm{Ti}$ to $\mathrm{Nb}_{3} \mathrm{Sn}$, with peak field in the coils of $11.4 \mathrm{~T}$ [17].

The $\mathrm{Nb}_{3} \mathrm{Sn}$ technology applied to accelerator magnets allows to increase the field from the $8 \mathrm{~T}$ reached in the LHC dipole mass production towards the limit of $15 \mathrm{~T}$, as shown in Fresca2 [21] and MDPCT1 [22] dipoles, both breaking the $14 \mathrm{~T}$ barrier in the past decade. In a circular accelerator the particle energy is proportional to the main dipole field and to the total length of the main dipoles, and therefore more field gives more energy. However, the relation between the peak field of the IR quadrupoles and the accelerator luminosity is much more complex [14]. For an upgrade of an existing accelerator, as the LHC, additional constraints are given by the tunnel geometry (diameter, and size of the straight sections dedicated to the IR) and quadrupoles with peak field $\geqslant 11.4 \mathrm{~T}$ were the only way to achieve the $150 \mathrm{~mm}$ aperture target. An higher peak field, in the range of 13-14 T, would have marginally increased the performance, at the price of a much larger cost (more conductor) and risk.

In fact, large aperture $\mathrm{Nb}_{3} \mathrm{Sn}$ quadrupoles were developed in the USA since 2004 [23-25], firstly with $90 \mathrm{~mm}$-aperture magnets (LARP-TQ, $10.3 \mathrm{~T}$ peak field) with two different mechanical structures, and successively with a $120 \mathrm{~mm}$ aperture magnet (LARP-HQ [26], $10.8 \mathrm{~T}$ peak field) and with scaling of the TQ short model to a $3.7 \mathrm{~m}$ long magnet (LARPLQ [27, 28]). The final solutions adopted for the HL-LHC quadrupoles, named MQXF [29-31], heavily rely on the R\&D carried out in LARP, and on the massive conductor development program [32] for $\mathrm{Nb}_{3} \mathrm{Sn}$ launched by the US Department of Energy in 2000.

LARP made use of high- $j_{\mathrm{c}} \mathrm{Nb}_{3} \mathrm{Sn}$ wires, with non-Cu critical current density of $\sim 1500 \mathrm{~A} \mathrm{~mm}^{-2}$ at $15 \mathrm{~T}$ and $4.2 \mathrm{~K}$. Continuous efforts are ongoing in the community to increase $j_{c}$; for the HL-LHC triplet, a conservative specification of $1280 \mathrm{~A} \mathrm{~mm}^{-2}$ at $15 \mathrm{~T}$ and $4.2 \mathrm{~K}$ has been adopted, to avoid significant rejection of production and to optimize the cost.

Accelerator dipoles based on $\mathrm{Nb}_{3} \mathrm{Sn}$ conductor were considered for LHC, with a $10 \mathrm{~T}$ CERN-ELIN dipole magnet [33] built at the end of the 80s. In the 90s, the $11 \mathrm{~T}$ barrier was breached with the graded dipole magnet at University of Twente [34], and a bore field above 13 T was reached with D20 dipole magnet [35] built in LBNL. The HD2 dipole magnet 
[36] reached similar values as D20 in 2010, and 5 years later a new record of $14.5 \mathrm{~T}$ bore field were reached in a much larger aperture in FRESCAII dipole magnet at CERN [21]. The MDPCT1 dipole, developed in FNAL and based on a four layer sector coil, also broke the $14 \mathrm{~T}$ barrier in a full accelerator-quality configuration [22]. All these models had a length of the order of 1-2 m. Moreover, the experience on racetrack coils (HD1 in LBNL [37] and ERMC at CERN [38]) show the conductor can tolerate peak fields as high as $16 \mathrm{~T}$.

A special challenge of the $\mathrm{Nb}_{3} \mathrm{Sn}$ magnets in HL-LHC is the high level of stress in the coils induced by the electromagnetic forces, that is about twice what can be found in the $8 \mathrm{~T} \mathrm{LHC}$ dipoles. In the HL-LHC triplet the stress in the coil can reach $150 \mathrm{MPa}$; above this limit a significant degradation of the $\mathrm{Nb}_{3} \mathrm{Sn}$ perfomance may occur [39, 40]. Similar challenges are present for the $11 \mathrm{~T}$ dipole magnet, $\mathrm{a} \mathrm{Nb}_{3} \mathrm{Sn}$ dipole magnet also planned to be installed in the HL-LHC, presently under construction at CERN $[31,41]$. Both the $11 \mathrm{~T}$ and the MQXF magnets will provide precious information for the possible application of $\mathrm{Nb}_{3} \mathrm{Sn}$ technology to future colliders [42, 43].

The IR magnets include not only the triplet, but also a separation and a recombination dipole, in the 5-6.5 $\mathrm{T}$ peak field range, and three types of correctors in the 2-4 $\mathrm{T}$ peak field range. For these magnets the $\mathrm{Nb}_{3} \mathrm{Sn}$ technology is not required, and they all rely on $\mathrm{Nb}-\mathrm{Ti}$. However, each of these magnets present interesting challenges to the superconduting technologies for accelerators. The 5.6 $\mathrm{T}$ bore field separation dipole [44-51], referred to as D1, has an unprecedented level of accumulation of coil stress in the midplane for $\mathrm{Nb}-\mathrm{Ti}$ magnets $(100 \mathrm{MPa})$ due to the combination of very large aperture and large current density. Reaching a preload level required to balance these forces is non trivial: here, a structure based on a full support from the iron yoke has been adopted. The $4.5 \mathrm{~T}$ bore field recombination dipole [52-55] has a special challenge in achieving a good field quality; the design is based on a similar concept developed for a D2 upgrade in [56], where an left-right asymmetric coil compensates the magnetic coupling between the apertures. Asymmetric $\mathrm{Nb}-\mathrm{Ti}$ coils for particle accelerator magnets were also succesfully manufactured for the combined function JPARC magnets [57].

The IR magnets include three types of correctors. The nested dipole corrector providing $2.1 \mathrm{~T}$ in both horizontal and vertical directions is based on a novel mechanical structure [58-60], with a double collaring and a mechanical lock between the horizontal and vertical dipole coils. This design presents a special interest since the double collaring allows intercepting forces and stresses in the coil with an intermediate mechanical structure, as in the stress management concept. When both apertures are powered, the bore field is $3.4 \mathrm{~T}$ and the coil peak field is $4.3 \mathrm{~T}$.

The double aperture $2.6 \mathrm{~T}$ dipole corrector is based on a canted $\cos \theta$ design [61-63], first proposed at the end of the 1970s [64], later industrialized in the US for several applications [65] and proposed also for high field magnets [66]. This magnet design, also quoted in the literature as titled solenoid or double helix, will be used for the first time in a CERN accelerator.

Finally, the high order correctors [67-73] rely on a superferric design (see for instance FAIR magnets [74] and SLHC prototypes [75], based on the same concept).

An important feature of the accelerator magnets is the compactness. With respect to experimental magnets in high energy physics, to MRI and to NMR solenoids, and to magnetic systems needed for fusion, accelerator magnets must be extremely compact, requiring very high current densities: the overall current density, defined as the current over the insulated coil, is of the order of $400 \mathrm{~A} \mathrm{~mm}^{-2}$, i.e. 4-10 times larger than in the previously quoted devices [1-3]. This introduces a series of challenges that are specific to these magnets [76], as the already mentioned large accumulation of stress, but also the risk of training, and instabilities in the superconductor for the $\mathrm{Nb}_{3} \mathrm{Sn}$ case [77, 78], and a challenging protection system. For the HL-LHC triplet, a novel protection scheme, called Coupling Loss Induced Quench (CLIQ) has been developed at CERN and has been adopted as the baseline [79].

HL-LHC was endorsed as the flagship project of CERN in 2013, and thereafter approved and financed in June 2016. Since then, the final phases of the conceptual and engineering design have been completed, and the prototyping phase is now coming to an end. The production shall last 5 years, and the magnets installation is foreseen starting in 2025. A special feature of this project, compared to an entirely new collider, is the relatively low number of magnets, that gives very little possibility of optimizing or fine tuning the design during the production.

The six types of magnets are shared by six international collaborations, which contribute to a large fraction of the work inkind, with CERN and a consortium of US laboratories (LBNL, FNAL and BNL) building the triplet, KEK in Japan building the D1 separation dipole, INFN-Genova in Italy the D2 recombination dipole, CIEMAT in Spain the nested correctors, IHEP in China the canted $\cos \theta$ corrector and INFN-LASA-Milano in Italy the superferric correctors. CERN takes care of integrating the correctors in the cold masses of the main magnets.

The aim of this paper is to review the main design choices, both in terms of layout and in terms of magnet technology, and to describe in detail the validation of the design via the model and prototype manufacturing and via the power tests; the design of the cold masses and of the cryostat are not discussed in this paper.

The six different magnets are treated in separate sections, each one having a short description of the accelerator requirements and of the design choices, followed by the design validation via power tests. Additional paragraphs are dedicated to the design changes since the beginning of the project, to the open issues and setbacks, and to the project timeline. The six sections are preceded by a general one, dedicated to a review of the types of magnets, to the choices made for the layout, and to the main magnet design parameters. 


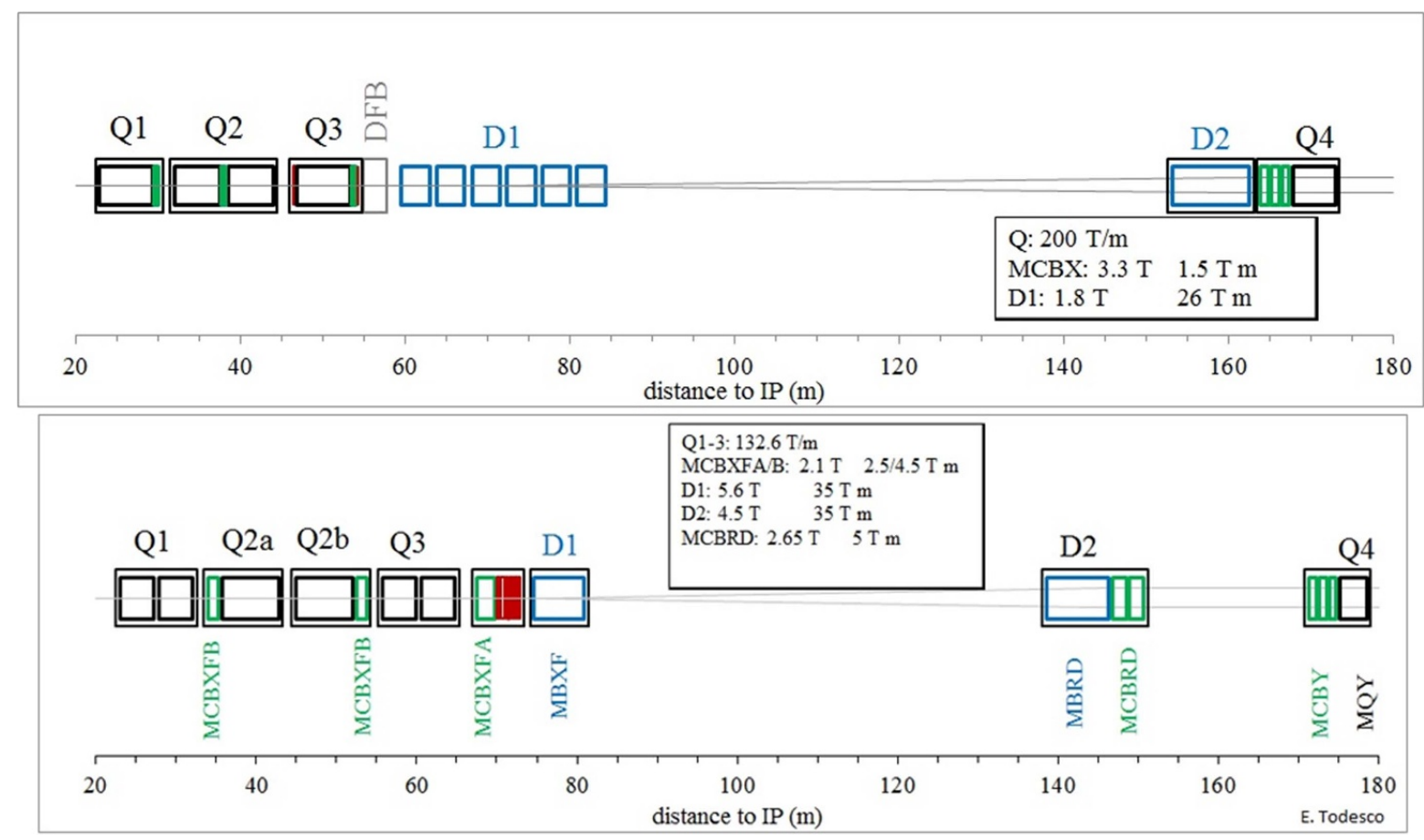

Figure 1. The lay-out of the LHC interaction region (upper part) and of the HL-LHC interation region (lower part). Thick boxes are magnets, and thin ones are cryostats.

\section{Interaction region magnets layout and features}

\subsection{Lay-out and magnet requirements}

The HL-LHC project requires six different types of magnets in the IRs around the two physics experiments ATLAS and CMS:

- three types of main magnets: separation (MBXF, also known as D1) and recombination (MBRD, aka D2) dipole magnets, and triplet of quadrupole magnets (MQXFA/B, aka Q1/Q2/Q3);

- two types of orbit correctors: single aperture nested correctors MCBXFA/B (aka the nested correctors) and double aperture correctors MCBRD (aka D2 correctors);

- one skew quadrupole corrrector MQSXF and four types of nonlinear (higher than quadrupole) correctors: sextupole MCSXF, octupole MCOXF, decapole MCDXF and dodecapole MCTXF (installed in normal and skew configurations). These different magnets are sharing the same design concept.

The lay-out of the LHC and of the HL-LHC are compared in figure 1 .

Thanks to the $\mathrm{Nb}_{3} \mathrm{Sn}$ technology, using a high-current density strand based on Rod Restack Process ${ }^{\circledR}$ (RRP), see section 3.3 for details, the peak field is increased from $\sim 8$ to $\sim 12 \mathrm{~T}$, and the triplet aperture can be doubled keeping the total length increased within $40 \%$ (from 25 to $35 \mathrm{~m}$ ). The magnetic length is $8.4 \mathrm{~m}$ for Q1/Q3 and $7.15 \mathrm{~m}$ for Q2a and Q2b [15-17]. The US collaboration, in charge of Q1/Q3, decided to split the magnet in two $4.2 \mathrm{~m}$ long parts, to minimize the risks due to the length increase with respect to the $3.7 \mathrm{~m}$ long magnet $\mathrm{LQ}$, the longest $\mathrm{Nb}_{3} \mathrm{Sn}$ accelerator magnet manufactured by LARP [27, 28].

To make room for the additional $10 \mathrm{~m}$ of the triplet, and for the $20 \mathrm{~m}$ needed to install crab cavities between D2 and Q4 [15, 16], two steps are taken: (a) the $25 \mathrm{~m}$ long resistive dipole D1 in LHC is replaced by a compact superconducting device (20 m saved), and (b) the separation/recombination dipoles integrated field is increased from 27 to $35 \mathrm{~T} \mathrm{~m}$, enabling a reduction of the D1-D2 distance.

In HL-LHC the orbit correctors of the IR magnets are located (as in the LHC [1]) close to each quadrupole, but their strength is increased from $2 \mathrm{~T} \mathrm{~m}$ to $2.5 \mathrm{~T} \mathrm{~m}$ and $4.5 \mathrm{~T} \mathrm{~m}$. Additional orbit correctors of $5 \mathrm{~T} \mathrm{~m}$ are located close to the $\mathrm{D} 2 \mathrm{mag}-$ net (they are not present in the LHC layout). The nonlinear correctors, containing up to dodecapole components, are gathered in a special module between the triplet and D1, referred to as corrector package. Skew dodecapole, and normal and skew decapoles are foreseen for HL-LHC while do not exist in the LHC. In the first baseline, a new $90 \mathrm{~mm}$-aperture Q4 was also present; a short model has been developed by CEA, Saclay [80], and will be tested in 2021. Two prototypes are being developed in the European industry with EU support in the QUACO initiative.

The beam dynamics requirements on the magnetic lattice are satisfied via the selection of a design for each magnet type. In the next section we will summarize the main features of the selected designs, focusing on the aspects that we consider the most relevant in terms of superconducting technology. 


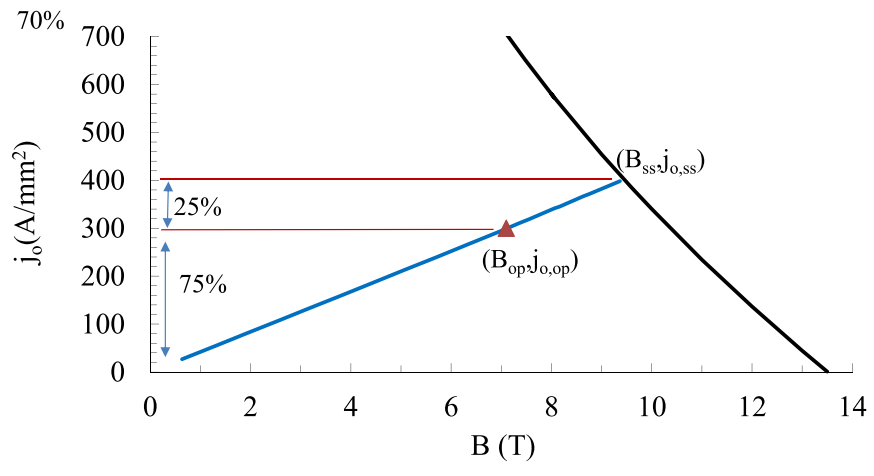

Figure 2. Magnet loadline and conductor critical surface at a given operating temperature; short sample condition (ss) and operational condition (op) are shown for a case of operational current at 0.75 loadline fraction.

\subsection{Peak field and loadline fraction}

The first critical parameter we consider is the peak field in the superconducting coil. We use this quantity rather than the bore field since the HL-LHC magnets include not only dipoles, but also quadrupoles and higher order multipoles. Moreover, we use this quantity rather than the maximum field in the magnet aperture (i.e. the gradient times the aperture radius for a quadrupole) since the peak field sets, for a given temperature, the limits to the maximum current density in the superconductor. At $1.9 \mathrm{~K}, \mathrm{Nb}-\mathrm{Ti}$ can carry sizeable current densities to build accelerator magnets up to $8-10 \mathrm{~T}$, and $\mathrm{Nb}_{3} \mathrm{Sn}$ up to $14-16 \mathrm{~T}$.

The second critical parameter is the loadline fraction, defined as the ratio between the operational current and the maximum current tolerable to the superconductor in the magnet following the loadline (also referred to as short sample current limit, see figure 2). This quantity is the condition in which the current density and the peak field in the coil reach the superconductor critical surface at the given temperature. The loadline fraction is therefore a number smaller than one, and the difference between one and the loadline fraction is defined in the literature as loadine margin. In figure 2 we show the case of a $\mathrm{Nb}-\mathrm{Ti}$ magnet, with $400 \mathrm{~A} \mathrm{~mm}^{-2}$ short sample overall current density and operational overall current density at $300 \mathrm{~A} \mathrm{~mm}^{-2}$, with a 0.75 loadline fraction or a $25 \%$ margin on the loadline. The loadline fraction is one of the most discussed parameters in the design phase, since one has to find a compromise between large margin to guarantee a low rejection rate of the magnet production, and a small margin to limit the cost and the size of the coils and, thereby, of the magnets.

In figure 3 we show for each type of HL-LHC IR magnet the loadline fraction vs the peak field in the coil at $1.9 \mathrm{~K}$. For the most challenging magnet, i.e. the triplet, we have a $11.4 \mathrm{~T}$ peak field, requiring the use of $\mathrm{Nb}_{3} \mathrm{Sn}$, and a loadline fraction of 0.78. LARP quadrupoles had a peak field of $10.4 \mathrm{~T}$ (TQ) and 11.0 T (HQ), and a loadline fraction of $\sim 0.80[24,26]$. Note that LHC dipoles, relying on $\mathrm{Nb}-\mathrm{Ti}$ conductor, operated until now at 0.80 loadline fraction in RunII (corresponding to $6.5 \mathrm{TeV}$ proton energy), with the target of operating at $0.86(7 \mathrm{TeV})$ in RunIII $[1,3]$. The present triplet magnets $[18,19]$, also wound with $\mathrm{Nb}-\mathrm{Ti}$, have loadline fraction

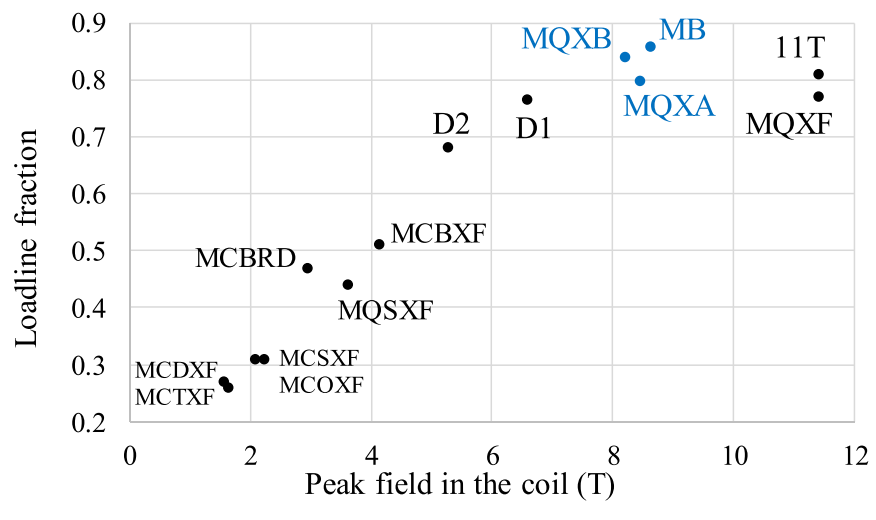

Figure 3. Peak field in the coil versus loadline fraction of HL-LHC IR main magnets and correctors, $11 \mathrm{~T}, \mathrm{LHC}$ main dipole (MB) and LHC IR quadrupoles (MQXA and MQXB).

at $7 \mathrm{TeV}$ between 0.80 and 0.84 , and have been operated at $6.5 \mathrm{TeV}$ at 0.74 and 0.78 respectively.

For the other magnets the peak field and the loadline fraction are lower (see figure 3), since the impact on cost and performance is lower, and one wishes to minimize the risk on these magnets.

- D1 has a $6.5 \mathrm{~T}$ peak field, with a 0.77 loadline fraction. Initially it was set at 0.75 , and later was increased to reduce the magnet length to fit the KEK vertical test station.

- D2 has a $5.3 \mathrm{~T}$ peak field, with a more conservative 0.68 loadline fraction.

- Corrector magnets have a peak field ranging from 1.5 to 3.4 T, and have a loadline fraction below 0.5.

We also give the position of the $11 \mathrm{~T}$ dipole $[15,16,31,41]$, that has 0.81 loadline fraction, and the same peak field. All these values refer to nominal field, corresponding to $7 \mathrm{TeV}$ operation. Following the LHC paradigm, the target of operation at $7.5 \mathrm{TeV}$ (named ultimate current) should be possible for all hardware, without any engineering margin. All considerations in this section will be done for nominal current. In the next sections dealing with the test results, the level of nominal and ultimate current will be shown in all plots.

\subsection{Current density and accumulated stress due to electromagnetic forces}

In the absence of iron, the magnetic field generated in the straight section of a saddle shape coil is proportional to the number of Ampere turns (number of turns of the coil times the current in the conductor). For superconducting magnets this equation is more adequate when written in terms of overall current density and coil width. For a typical sector coil of width $w$ (see figure 4) the dipolar field is proportional to the current density times the coil width

$$
B=\gamma j w
$$

with a constant $\gamma$ that for a $60^{\circ}$ sector is $6.9 \times 10^{-7} \mathrm{~T} \mathrm{~m} \mathrm{~A}^{-1}$ [81], and for a more realistic coil layout with a wedge to 


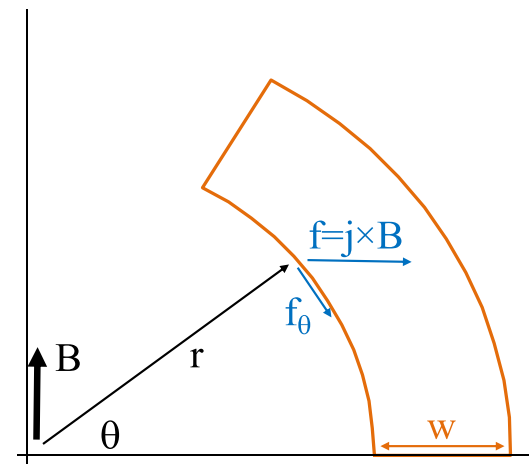

Figure 4. Dipole sector coil of inner radius $r$ and width $w$ (one quarter shown), and electromagnetic force on the coil edge assuming a field on the coil equal to the field in the centre.

optimize field quality is $6.6 \times 10^{-7} \mathrm{~T} \mathrm{~m} \mathrm{~A}^{-1}$. The third critical parameter we consider is the overall current density $j$, i.e. the Ampere turns divided by the area of the sector coil. Note that this overall current density is computed on the conductor cross-section, including insulation and voids between strands. The term engineering current density, also used in the literature, usually refers to current density over the conductor without insulation. For a quadrupole one has a logarithmic dependence of the gradient $G$

$$
G=\gamma j \ln \left(1+\frac{w}{r}\right)
$$

where $r$ is the inner radius of the coil sector, and for a $30^{\circ} \mathrm{sec}-$ tor the constant $\gamma$ has the same value as for the dipole case [82]. For these equations and constants, overall current densities are expressed in $\mathrm{A} \mathrm{m}^{-2}$.

Note that in the following we will use the more practical units $\mathrm{A} \mathrm{mm}^{-2}$. With these current density units, the constants become $6.9 \times 10^{-4} \mathrm{~T} \mathrm{~mm} \mathrm{~A}^{-1}$ for a dipole, with the width expressed in $\mathrm{mm}$, and $0.69 \mathrm{~T} \mathrm{~m}^{-1}\left(\mathrm{~A} \mathrm{~mm}^{-2}\right)^{-1}$ for a quadrupole, with a gradient expressed in $\mathrm{T} \mathrm{m}^{-1}$. If the reader gets lost, we advice to use the international system units to carry all computations.

Typical overall current densites in superconducting accelerator magnets range between 300 and $600 \mathrm{~A} \mathrm{~mm}^{-2}$. Large current densites have the advantage of giving a cheaper and more compact device. The price one has to pay for high current densities is related to three different aspects. First, operating the supercondutors at higher current densities reduces the loadline fraction and generates higher risk of quenching. Second, high current densities increase the stress in the coil due to electromagnetic forces. Third, the magnet protection sets an upper limit of $\sim 1000 \mathrm{~A} \mathrm{~mm}^{-2}$ to the maximum overall current density. This is given by the balance between the energy density of the magnet over the winding and the heat capacity of the winding itself, that has to absorb the magnet energy during a quench without damaging (melting) the coil. This limit is typical of main accelerator magnets (i.e. dipole and quadrupole magnets) where an energy extraction system for each magnet is not viable and/or affordable. For $\mathrm{Nb}_{3} \mathrm{Sn}$ magnets there is a fourth issue related to high current densities: the conductor instabilities [77, 78].

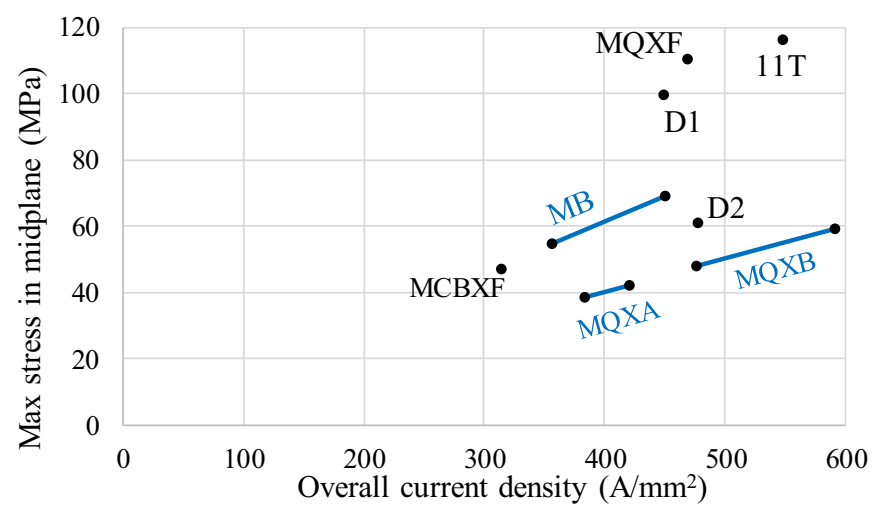

Figure 5. Overall current density versus maximum midplane stress (in absence of structure and coil deformations) for HL-LHC IR main magnets, $11 \mathrm{~T}$, LHC main dipole (MB) and LHC triplet (MQXA and MQXB).

The fourth critical parameter we consider is the stress accumulation in the midplane. We recall that the force per unit of volume is given by the current density times the magnetic field in the coil. For a sector dipole coil, we carry out a first order estimate of the midplane stress with the assumption that the field at the inner edge of the coil is equal to the field in the centre of the magnet, and that the component tangent to the aperture radius accumulates to the midplane (see figure 4). One can then integrate the azimuthal component of the force over the sector inner radius, obtaining the midplane stress at the aperture radius $r$

$$
\sigma_{r}=\int_{0}^{\pi / 3} j B r \sin \theta d \theta=-\frac{1}{2} j B r
$$

where we considered a sector coil of $60^{\circ}$, canceling the sextupole component. For a quadrupole sector coil of $30^{\circ}$, in the same hypothesis one gets

$$
\sigma_{r}=-\frac{1}{4} j G r^{2}
$$

These simple estimates already gives a warning for the HL-LHC magnets: if the same parameters for field/gradient and current density were used, the $150 \mathrm{~mm}$ aperture gives a factor two increase with respect to the LHC IR magnets (70 $\mathrm{mm}$ aperture) and almost three with respect to the LHC main magnets (56 $\mathrm{mm}$ aperture).

There is an additional aspect to consider: the peak stress at the midplane inside the coil is larger than the stress at the bore radius $r$ : this effect is particularly large for large coil widths, as discussed in the appendix A. In figure 5 we plot the overall current density vs the maximum accumulated stress in the midplane, using the more refined estimate given in the appendix A and based on $[83,84]$ for the main HL-LHC IR magnets. Note that structure deformation is neglected in this analytical estimate. We also give the values for the $11 \mathrm{~T}$, and for the LHC main dipole (main bending, aka MB) and triplet magnets (MQXA and MQXB). For the LHC MB and for the LHC triplet magnets, one has two points since the coil is graded with higher overall current density in the lower field, outer coil layer. 
Note that for all HL-LHC IR magnets the stress accumulation in the midplane is larger than the stress accumulation in the radial direction, that is proportional to the magnetic pressure via a constant that is $\sim 1.5$. The constant is due to the nonuniform pattern of radial forces induced in a dipole (it is one for a solenoid). This is why here the radial pressure is not considered a design parameter. For other cases, namely for dipole magnets with fields $>15 \mathrm{~T}$, the stress accumulation in the radial direction can become dominant. Whereas the stress accumulation in the midplane can always be reduced by using smaller current densities and larger coils, the stress accumulation in the radial direction is mainly given by the level of bore field.

We can make the following considerations:

- The HL-LHC triplet has a current density similar to the outer layer of the LHC dipole magnets. Compared to the inner layer of the LHC dipole, the triplet has $30 \%$ larger current density. Moreover, the accumulated stress in the midplane is about twice that of the LHC dipole magnets. These aspects represent the main challenges (and advancements) with respect to the $\mathrm{Nb}-\mathrm{Ti}$ technology.

- The separation/recombination dipole magnets have similar current densities to the HL-LHC triplet. The accumulated stresses are much more critical for D1, whose level is around $100 \mathrm{MPa}$, close to the MQXF and to the $11 \mathrm{~T}$. Therefore, even if the field is lower than for the LHC main dipoles, D1 is more challenging from the point of view of accumulated midplane stress. On the other hand, D2 and the nested correctors do not show significantly larger challenges with respect to the LHC main dipole.

- The $11 \mathrm{~T}$ has a midplane stress close to the triplet: looking at equation (3), the smaller aperture is compensated by the factor of two between dipoles and quadrupoles, plus a $20 \%$ larger current density.

\subsection{Energy density and protection}

The fifth critical parameter we wish to comment on is the overall energy density in the coil. This is defined as the ratio between the magnet stored energy and the volume of the insulated cable. This parameter is relevant to protection since in case of no energy extraction, the magnetic energy has to be dissipated in the coil, increasing its temperature and determining the hot spot temperature (maximum temperature in a coil during quench). An intrinsic limit to the energy density is $\sim 0.5 \mathrm{~J} \mathrm{~mm}^{-3}$, which correponds to the order of magnitude of the enthalpy needed to bring a typical insulated coil from $1.9 \mathrm{~K}$ to room temperature [85]. Obviously, the precise value of this limit depends on the fraction of insulation, of voids or of resin, and the ratio between the superconductor and the copper in the superconducting wire, which have not exactly the same enthalpy. The LHC dipole magnets and the LHC triplet have an energy density of $\sim 0.05 \mathrm{~J} \mathrm{~mm}^{-3}$, and in the case of HL-LHC we reach $\sim 0.10 \mathrm{~J} \mathrm{~mm}^{-3}$ for the triplet.

The sixth (and last) critical parameter is the fraction of copper in the strand. In the triplet we have a copper/non copper ratio of $1.2: 1$, i.e. $55 \%$ of the strand is composed by $\mathrm{Cu}$.
This ratio is lower than in the LHC dipoles $(62 \%$ and $66 \%$ in inner and outer layer respectively). This is done to reduce the loadline fraction, i.e. to give more margin for the magnet operation. The price to pay is that in case of a quench, the current density in the copper will be larger, resulting in faster conductor heating.

A global figure of merit of the protection is the time allowed to detect the quench and bring the whole coil to the resistive state, keeping the final temperature of the coil below room temperature in all its parts. In the LHC dipole this time is $\sim 100 \mathrm{~ms}$ for the outer layer, and $\sim 200 \mathrm{~ms}$ [85]. In the HL-LHC triplet this time is $40 \mathrm{~ms}$. This gives a quantitative idea of the unprecedented parameters that are being explored in the HL-LHC triplet in terms of protection. On the other hand, the other $\mathrm{Nb}-\mathrm{Ti}$ magnets of HL-LHC do not pose particular challenges in terms of protection with respect to previous projects.

\subsection{Summary table}

We conclude this section by giving a complete list of the main magnet and dipole corrector parameters in table 1 . The detail of the design for each magnet shall be discussed in the next sections. The parameters of the skew quadrupole and of the high order correctors are given in section 8 .

\section{The triplet}

\subsection{Accelerator requirements}

The triplet is a set of three quadrupoles, with equal and opposite gradients, alternating in the sequence +-+ positive - negative - positive. Their optical function is to reduce the beam size in the cell arc by more than one order of magnitude. During the acceleration, the gradient of the triplet increases proportionally to the beam energy, as the field of the main dipoles. When the beam is injected, the optical functions in the IP are large ( « unsqueezed »), i.e. the beam size is comparable to its value in the arcs. Morevoer, collisions in the IP are avoided through a small but sizeable (few millimeters) separation generated by the orbit correctors. After the beam reaches the maximum energy, the separation is removed, and the optical functions are squeezed in the IP. Note that the squeeze is done via a change of the gradient of the matching quadrupoles (Q4 to Q7): during this operation the triplet gradient is kept at its nominal value.

Both in the LHC and in the HL-LHC, the triplet is made of four magnets, since the second unit is about twice as long as the first and the third, and therefore is usually split in two. In the HL-LHC, the four magnets are in a series on the same circuit with the proper order of the poles to guarantee the alternance of polarity. There is a trim on the first and last quadrupole to allow precise measurements of the optical functions around the experiments.

When the beams are in collision mode, the beam dynamics of the whole LHC is dominated by the triplet field multipolar errors. For this reason there are stringent requirements on the multipoles of the triplet at nominal energy. On the other 


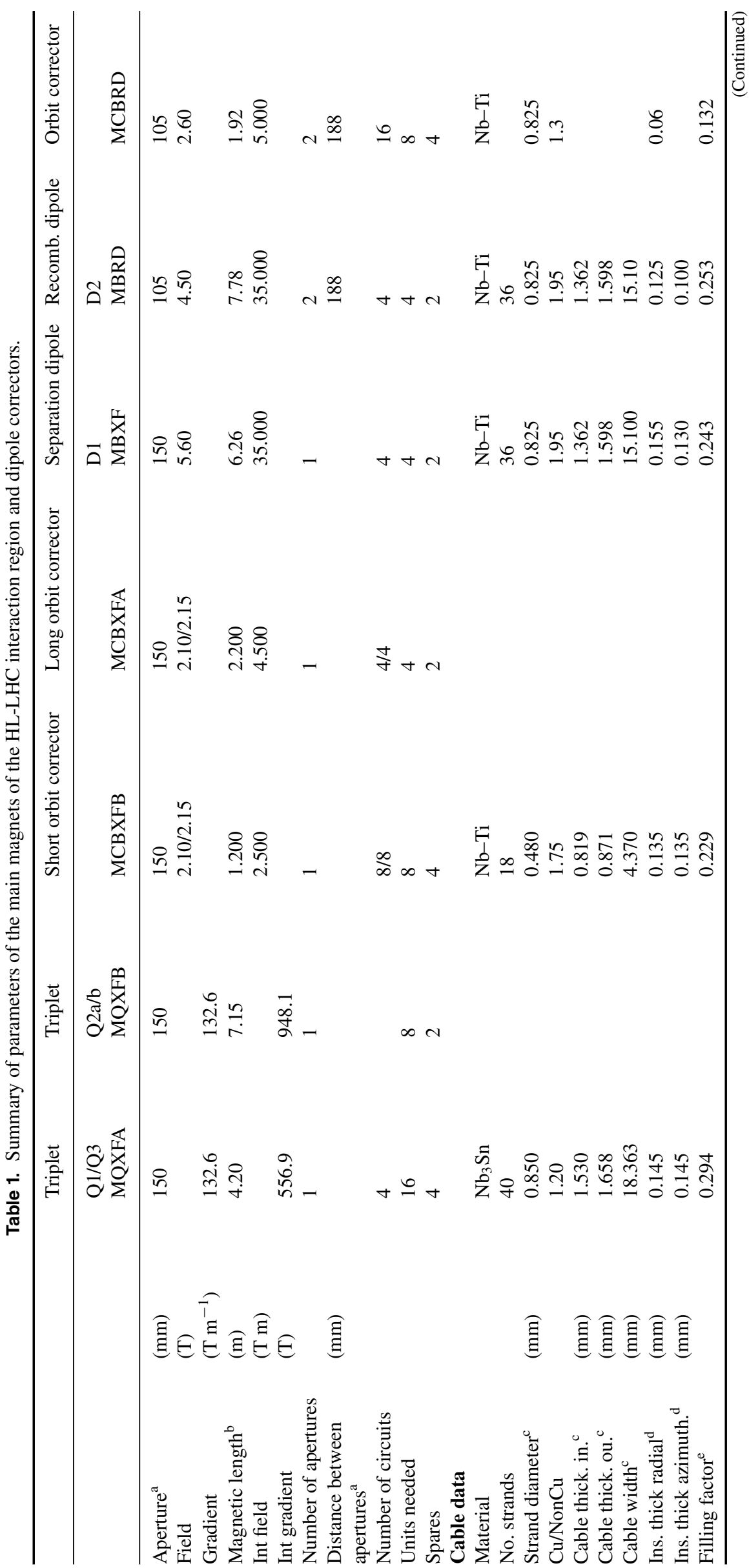




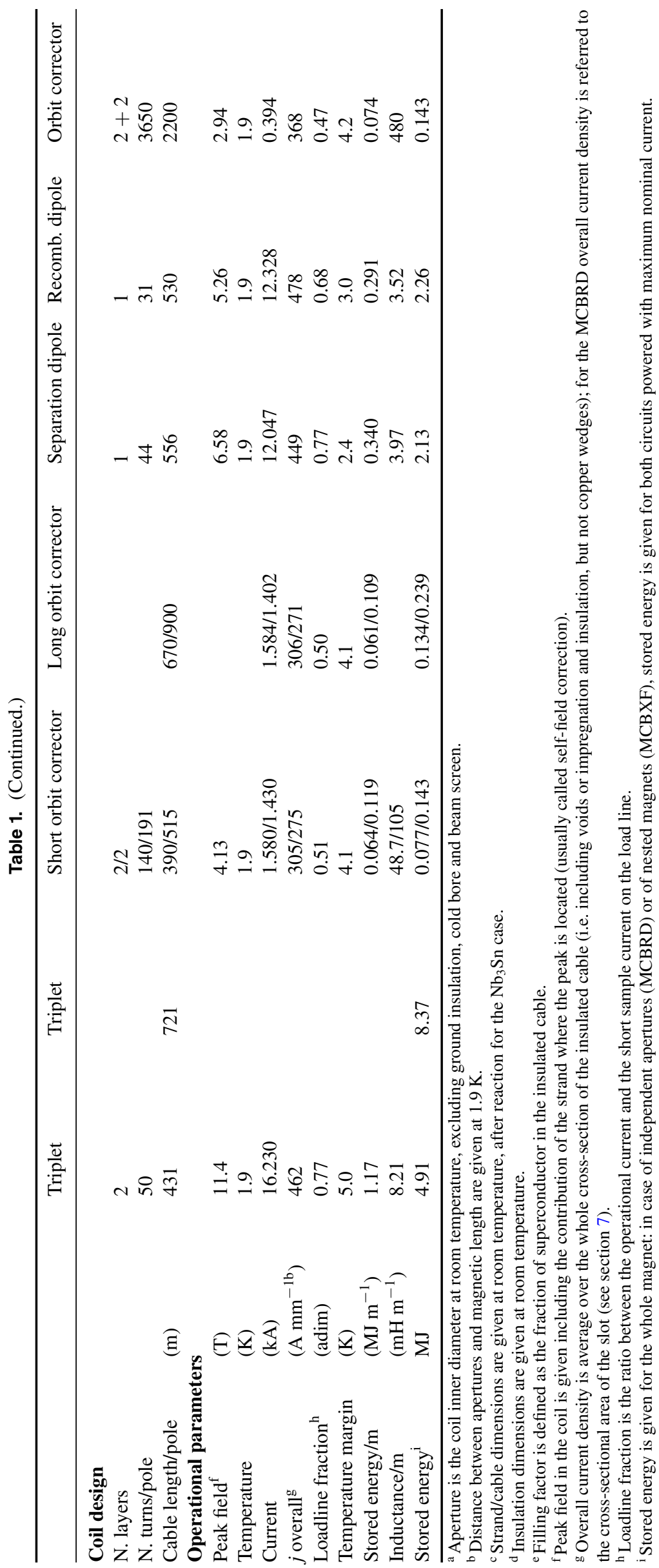




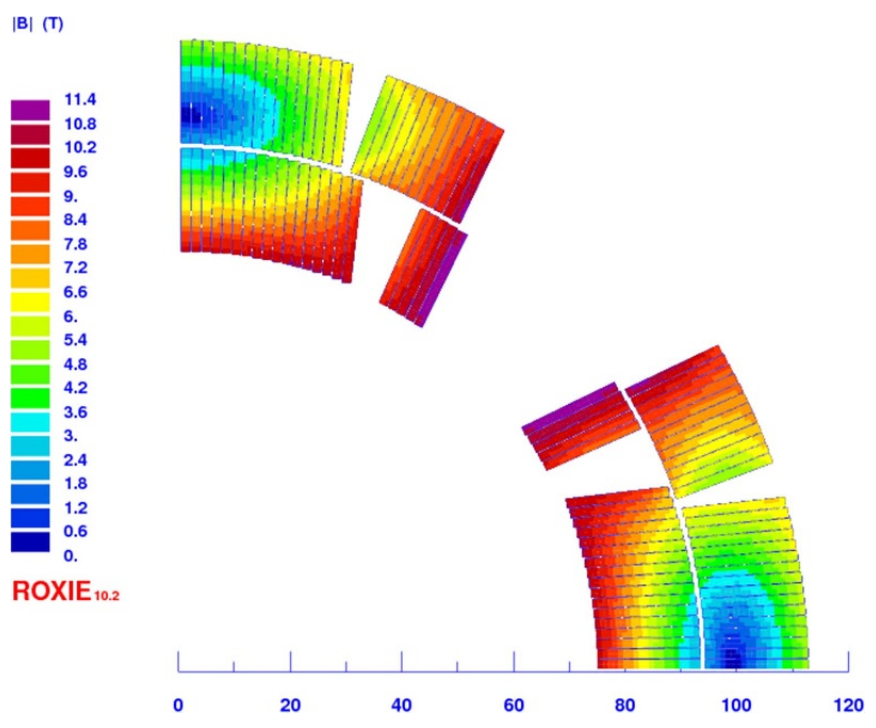

Figure 6. MQXF cross-section, field map at nominal current.

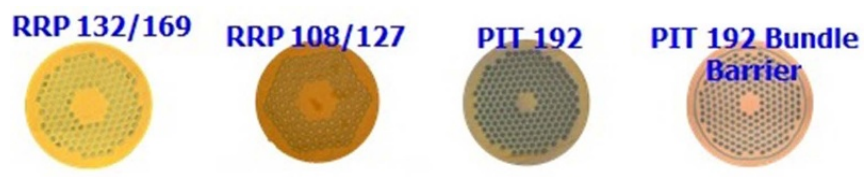

Figure 7. Comparison of strand layout.

a accelerator-like field quality at nominal current. As in the $\mathrm{HQ}$, the alignment between the last cable of the inner block and the pole of the outer block is imposed, to avoid cable torsion in the layer jump. The only difference with respect to HQ is the scaling of the conductor width from 15 to $18 \mathrm{~mm}$, to match the increased aperture. This is done by increasing the strand diameter from $0.8 \mathrm{~mm}$ to $0.85 \mathrm{~mm}$, and increasing the number of strands from 35 to 40 . Note that operational current was revised to $16.23 \mathrm{kA}$ from initial values of $16.47 \mathrm{kA}$, and ultimate current from $17.89 \mathrm{kA}$ to $17.50 \mathrm{kA}$ to better match the achieved gradient. In previous literature one can find the higher values.

\subsection{Design features: strand and conductor}

The conductor development program of US-DOE [32] focussed on the RRP $\mathrm{Nb}_{3} \mathrm{Sn}$ wire produced by OST. The LARP strand workhorse was a $0.7 \mathrm{~mm}$-diameter RRP wire (for TQ) and a $0.8 \mathrm{~mm}$-diameter RRP wire (for HQ), based on the same 54/61 layout, reaching crititcal current values in the superconductor up to $3000 \mathrm{~A} \mathrm{~mm}^{-2}$ at $12 \mathrm{~T}, 4.2 \mathrm{~K}$. Note that 54/61 means that there are 61 subelements, and 54 of them are $\mathrm{Nb}_{3} \mathrm{Sn}$, the other being $\mathrm{Cu}$ (see figure 7). This corresponds to filaments of $\sim 70 \mu \mathrm{m}$ diameter. A 108/127 layout was also developed and used for HQ, having a filament diameter of $\sim 50 \mu \mathrm{m}$. Layouts involving more filaments, as 132/169, were used in the MQXF short model program; eventually the 108/127 strand was adopted for the project, showing ability to reach target performance and having a lower cost.

For MQXF, the minimal critical current requirement is $1280 \mathrm{~A} \mathrm{~mm}^{-2}$ at $15 \mathrm{~T}$ and $4.22 \mathrm{~K}$. This specification corresponds to values $20 \%$ below the $3000 \mathrm{~A} \mathrm{~mm}^{-2}$ current density reached in LARP strands at $12 \mathrm{~T}$ and $4.2 \mathrm{~K}$. The RRR (Residual Ratio Resistivity, i.e. the ratio between the resistivity of the $\mathrm{Cu}$ in the strand at room temperature and at $1.9 \mathrm{~K}$ ) minimal requirement is 150 in the strand and 100 in the cable [86]. The cable degradation of critical current with respect to the unrolled strand has to be smaller than $5 \%$.

CERN also supported an effort to develop a second provider based on a different technology [87], namely the power in tube (PIT) strand by Bruker, with 192 subelements (see figure 7). It has been used for the construction of two short models, the second one having a different strand layout including a bundle barrier.

\subsection{Design features: coil manufacturing}

Coil insulation and manufacturing follow the same technology adopted by the LARP program; the insulation is a braided fiberglass. During the winding, a ceramic binder is used to give 


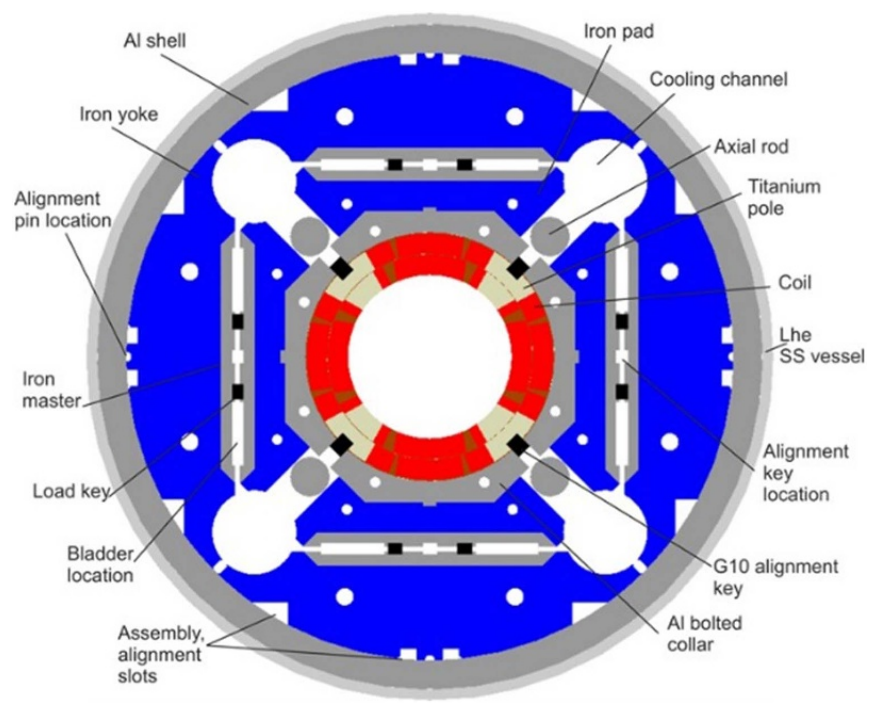

Figure 8. Cross-section of the triplet quadrupole.

rigidity to the coil; the inner layer is cured to allow winding the second layer above it. The two layers are then reacted with three $\sim 50 \mathrm{~h}$ long plateaux, the last one at $665^{\circ}$. This cycle was optmized to reach the best tradeoff in terms of $j_{\mathrm{c}}$, RRR, and reproducibility. The coil is then impregnated with CTD$101 \mathrm{~K}$, with a $5 \mathrm{~h}$ at $110{ }^{\circ} \mathrm{C}$ (curing) and $16 \mathrm{~h}$ at $125^{\circ} \mathrm{C}$ (postcuring).

\subsection{Design features: structure}

As mentioned earlier, the LARP structure based on Al shell and bladder and key loading, developed for TQS and HQ, has been selected [30] for MQXF (see figure 8). The coil pack is loaded in the $\mathrm{Al}$ shell at room temperature via bladders. The bladder pressure is an observable, so there is a very good control of the preload at room temperature. Once the desired value is obtained, keys are used to lock the structure and the bladders are removed. A azimuthal preload of $40-60 \mathrm{MPa}$ is imposed at room temperature on the coil midplane.

During cool down, the $\mathrm{Al}$ shell shrinks more than the iron and the coil, and this increases the preload in the coil to 80$120 \mathrm{MPa}$. The iron structure (in blue in figure 8) has open gaps according to the quadrupolar symmetry at room temperature and at $1.9 \mathrm{~K}$. This means that during cool-down, all the pressure given by the shell thermal contraction ends up on the coil, with the exception of the pole alignment key. This key was inserted in HQ to guarantee the alignment of the coils, partly intercepting the stress between the shell and the coil. According to the short model program results, the pole key could be not needed since magnetic measurements showed that coil alignment can be guaranteed by the assembly of the coil pack also in absence of interference with the pole key.

\subsection{Design features: protection}

The requirement on the hotspot temperature, i.e. the highest temperature reached in the conductor during a quench, is to be

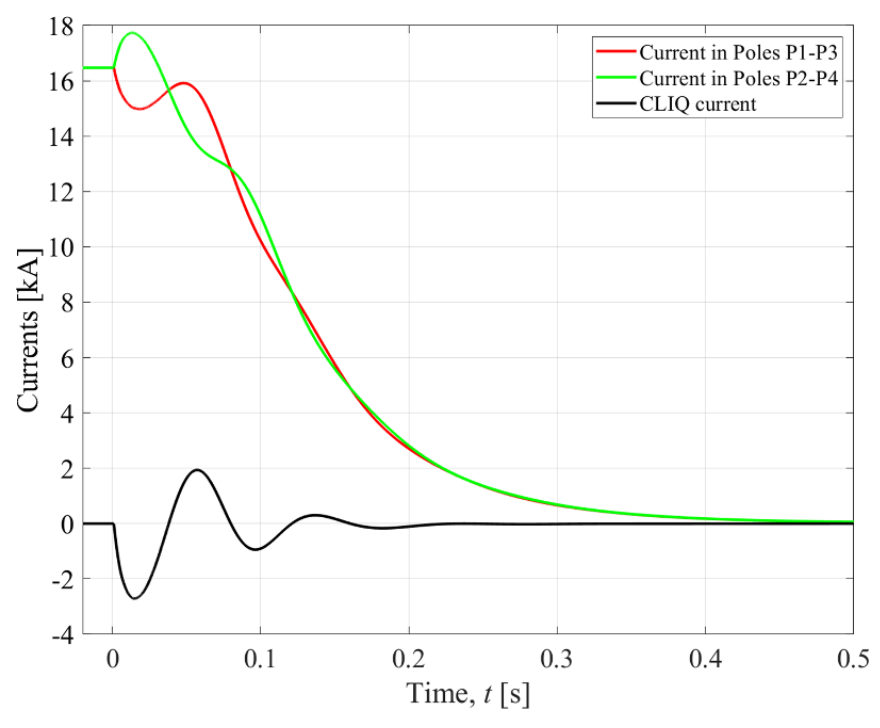

Figure 9. CLIQ discharge.

below $350 \mathrm{~K}$. This requirements is given to avoid that the coil resin goes above the glass transition. Two protection systems (outer layer quench heaters [88] and CLIQ [79]) are used to to satisfy this requirements.

Note that the condition on the hotspot must be guaranteed in case of two simultaneous failures of the protection system, i.e. either the failure of two quench heater firing or one quench heater and the CLIQ system. In case of no failures, the quench heaters and CLIQ guarantee a maximum hotspot temperature of $270 \mathrm{~K}$.

No energy extraction is present, as in all the main magnets of the HL-LHC IRs. The outer layer quench heater is made of a stainless steel strip, partially covered by $\mathrm{Cu}$, deposited on a $50 \mu \mathrm{m}$ polymide layer. The copper plating is done to focus the heat deposit in a series of $50 \mathrm{~mm}$ long heating stations along the magnet length: this design is required for magnets longer than $1 \mathrm{~m}$. In LARP, a $25 \mu \mathrm{m}$ polymide layer was used; in HL-LHC the polymide thickness was doubled to $50 \mu \mathrm{m}$ to increase the robustness of the insulation between the heaters and the coil. Each half-coil has two strips (low field and high field); the total of 16 strips per quadrupole are powered on eight independent circuits, guaranteeing the required redundancy.

CLIQ is a novel system, invented at CERN in 2014 [79], based on injecting a fast pulse of current in the coil to quench the magnet via the heating induced by eddy currents. The pulse amplitude is $1.5 \mathrm{kA}$, and the period is $\sim 70 \mathrm{~ms}$ (see figure 9).

\subsection{Production data: strand}

The strand production has been nearly completed [89]. The specification of $j_{\mathrm{c}}>1280 \mathrm{~A} \mathrm{~mm}^{-2}$ at $15 \mathrm{~T}, 4.22 \mathrm{~K}$ corresponds to $I_{\mathrm{c}}>331 \mathrm{~A}$, which is kept with a considerable margin ( $\sim 10 \%$ lower on the lowest values, and $\sim 15 \%$ lower than the average, see figure 10). There has been a change in the heat treatment procedure to improve RRR, that was initially at the edge of the 150 specification and after billet 40 is well above 


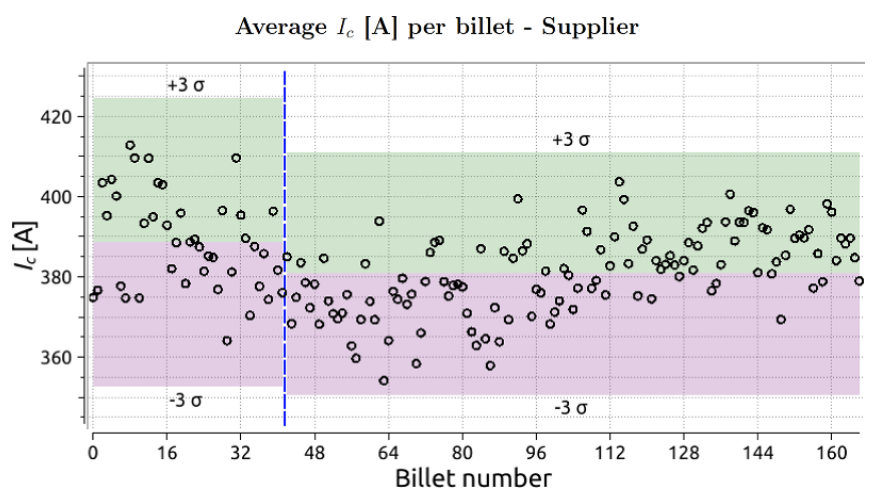

Figure 10. Critical current along production of RRP strand; the separation at billet 44 corresponds to the change in heat treatment.

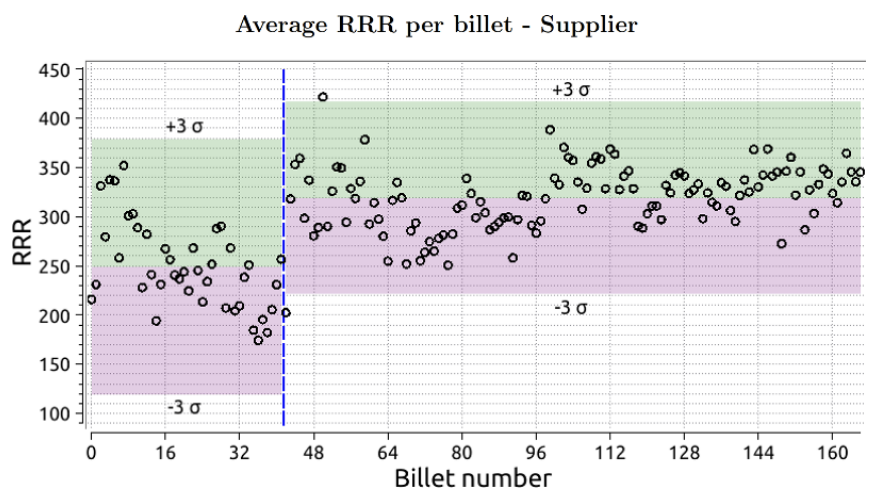

Figure 11. RRR along production of RRP strand; the separation at billet 44 corresponds to the change in heat treatment.

200 (see figure 11). The cable includes a stainless steel strip to control the dynamic effects; cabling is ongoing, showing no critical issues [90].

\subsection{Production data: coil size}

An essential ingredient of magnet manufacturing is reaching the nominal azimuthal dimension of the coils (see figure 12). A correct dimension of the coil allows reaching the prestress in the nominal position of the cable, i.e. achieving the required field quality and preload. A larger or smaller coil can be compensated via shim to achieve the target preload, but in this case the field quality is compromised. The second and even more important issue is the variation of the coil size along the magnet axis. If it becomes too large, this induces large variations of prestress that can degrade the cable or leave not enough prestress. The level at which the coil size can be controlled should be of the order of $\pm 0.1 \mathrm{~mm}$, that is twice the tolerance of the impregnation mould. Over an MQXF coil, whose azimuthal length is $\sim 90 \mathrm{~mm}$, this means a relative tolerance of the order of $\pm 0.1 \%$, that with a Young modulus of the order of $15 \mathrm{GPa}$ gives a precompression variation of $\pm 15 \mathrm{MPa}$. The short model coil production had a variation of average coil size within $\pm 0.2 \mathrm{~mm}$. Data relative to US and CERN prototypes show that the production is progressively achieving the required $\pm 0.1 \mathrm{~mm}$ target (see figure 13 ).

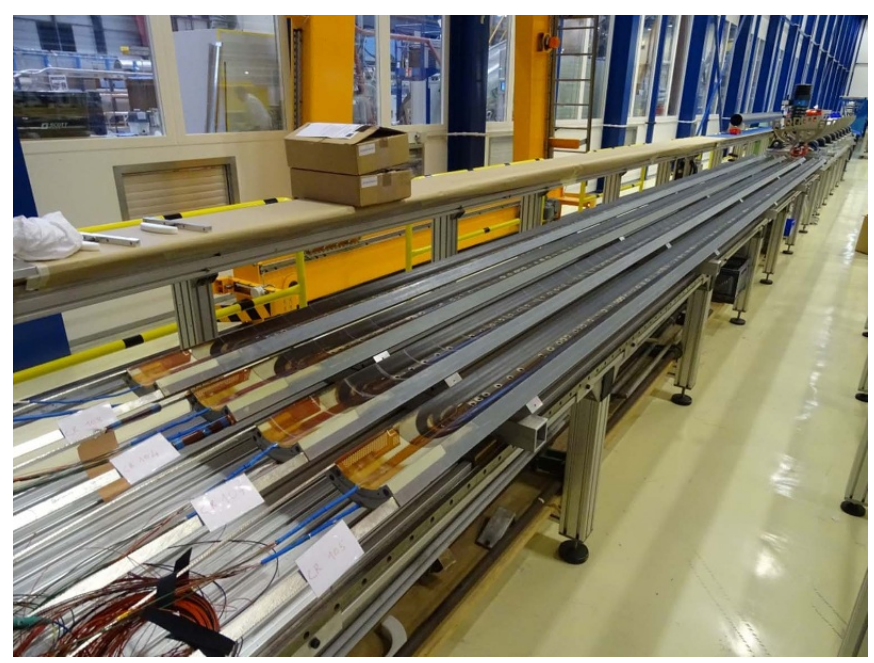

Figure 12. Four coils of MQXFB ready for assembly.

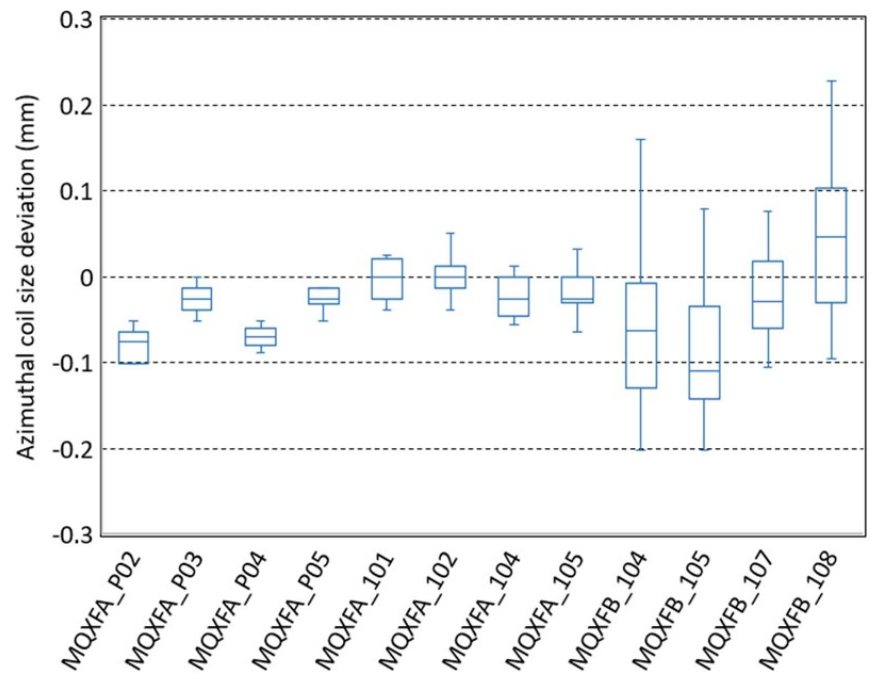

Figure 13. Coil size for US (MQXFA) and CERN (MQXFB) prototypes.

\subsection{Design validation via power tests: performance}

The short model program is a joint venture between US LARP and CERN, with coils sharing the same design. Out of a total of 34 manufactured coils for short models, 21 coils were tested in seven different assemblies, see table 2. Among these 21 coils, eight coils were made with the final RRP conductor (108/127); five coils were made with RRP with finer filaments (132/169); CERN manufactured coils with PIT 192 conductor, four of them with the initial layout and four with the bundle layout. In the same table we give the magnets were the coils were assembled and tested, the RRR measured during the cooldown via voltage taps, the critical current of the coil $I_{\mathrm{c}}$ in the magnet (sometimes called short sample current), based on the measurement of the strand properties of witnesses reacted with the coil, and of cable degradation. We then report if the nominal current has been reached; if not, the reached value in $\mathrm{kA}$ is given in brackets. We finally give in the last column the number of quenches needed to reach nominal current during the first powering. 
Table 2. Summary of manufactured coils and performance

\begin{tabular}{|c|c|c|c|c|c|c|c|}
\hline Coil id. & Strand & Made at & Assembled in & RRR & $I_{\mathrm{c}}(\mathrm{kA})$ & $\begin{array}{l}\text { Nominal } \\
\text { reached }(k A)\end{array}$ & $\begin{array}{l}\text { Number of quenches } \\
\text { to nominal current } \\
\text { during first powering }\end{array}$ \\
\hline 3 & RRP 108/127 & FNAL/BNL & MQXFS1 & 244 & 22.28 & $\mathrm{Y}$ & 1 \\
\hline 5 & RRP 108/127 & FNAL/BNL & MQXFS1 & 247 & 21.85 & $\mathrm{Y}$ & 1 \\
\hline 103 & RRP $132 / 169$ & CERN & MQXFS1 & 129 & 21.40 & $\mathrm{Y}$ & 3 \\
\hline 104 & RRP $132 / 169$ & CERN & MQXFS1 & 105 & 21.65 & $\mathrm{Y}$ & 1 \\
\hline 7 & RRP 108/127 & FNAL & MQXFS3a & 178 & & $\mathrm{Y}$ & 1 \\
\hline 8 & RRP 108/127 & FNAL/BNL & MQXFS3c & 182 & & $?$ & \\
\hline 105 & RRP 132/169 & CERN & $\begin{array}{l}\text { MQXFS3a } \\
\text { MQXFS3c }\end{array}$ & 155 & 21.55 & $\begin{array}{l}\mathrm{Y} \\
?\end{array}$ & 2 \\
\hline 106 & RRP 132/169 & CERN & $\begin{array}{l}\text { MQXFS3a } \\
\text { MQXFS3c }\end{array}$ & 160 & 21.55 & $\begin{array}{l}\mathrm{Y} \\
\mathrm{N}(15.0-16.0)\end{array}$ & 0 \\
\hline 107 & RRP 132/169 & CERN & $\begin{array}{l}\text { MQXFS3a } \\
\text { MQXFS3c }\end{array}$ & 135 & 21.33 & $\begin{array}{l}\mathrm{Y} \\
?\end{array}$ & 1 \\
\hline 203 & PIT 192 & CERN & $\begin{array}{l}\text { MQXFS5 } \\
\text { MQXFS6b }\end{array}$ & 80 & 20.84 & $\begin{array}{l}\mathrm{Y} \\
\mathrm{Y}\end{array}$ & $\begin{array}{l}0 \\
0\end{array}$ \\
\hline 204 & PIT 192 & CERN & $\begin{array}{l}\text { MQXFS5 } \\
\text { MQXFS6b }\end{array}$ & 88 & 20.86 & $\begin{array}{l}\mathrm{Y} \\
\mathrm{Y}\end{array}$ & $\begin{array}{l}1 \\
0\end{array}$ \\
\hline 205 & PIT 192 & CERN & MQXFS5 & 88 & 21.00 & $\mathrm{Y}$ & 1 \\
\hline 206 & PIT 192 & CERN & MQXFS5 & 90 & 20.54 & $\mathrm{Y}$ & 1 \\
\hline 108 & RRP 108/127 & CERN & MQXFS4 & 156 & 21.53 & $\mathrm{Y}$ & 0 \\
\hline 109 & RRP 108/127 & CERN & MQXFS4 & 154 & 21.84 & $\mathrm{Y}$ & 0 \\
\hline 110 & RRP 108/127 & CERN & MQXFS4 & 152 & 21.97 & $\mathrm{Y}$ & 0 \\
\hline 111 & RRP 108/127 & CERN & MQXFS4 & 151 & 22.00 & $\mathrm{Y}$ & 0 \\
\hline 208 & PIT 192 w b & CERN & MQXFS6a & 75 & 20.93 & $\mathrm{Y}$ & 3 \\
\hline 209 & PIT 192 w b & CERN & MQXFS6a & 75 & 20.90 & $\mathrm{Y}$ & 2 \\
\hline 210 & PIT 192 w b & CERN & $\begin{array}{l}\text { MQXFS6a } \\
\text { MQXFS6b }\end{array}$ & 90 & 20.51 & $\begin{array}{l}\mathrm{Y} \\
\mathrm{Y}\end{array}$ & $\begin{array}{l}0 \\
0\end{array}$ \\
\hline 212 & PIT 192 w b & CERN & $\begin{array}{l}\text { MQXFS6a } \\
\text { MQXFS6b }\end{array}$ & 95 & 20.64 & $\begin{array}{l}\mathrm{Y} \\
\mathrm{Y}\end{array}$ & $\begin{array}{l}0 \\
0\end{array}$ \\
\hline P02 & RRP 132/169 & FNAL/BNL & $\begin{array}{l}\text { MQXFAP1 } \\
\text { MQXFAP1b }\end{array}$ & NA & 21.7 & $\mathrm{Y}$ & 1 \\
\hline P03 & RRP 144/169 & FNAL & $\begin{array}{l}\text { MQXFAP1 } \\
\text { MQXFAP1b }\end{array}$ & NA & 22.0 & $\mathrm{Y}$ & 3 \\
\hline P04 & RRP 132/169 & FNAL/BNL & $\begin{array}{l}\text { MQXFAP1 } \\
\text { MQXFAP1b }\end{array}$ & NA & 21.6 & $\mathrm{Y}$ & 0 \\
\hline P05 & RRP 108/127 & FNAL & MQXFAP1 & NA & 22.0 & $\mathrm{Y}$ & 0 \\
\hline P06 & RRP 108/127 & FNAL & MQXFAP1b & NA & 21.9 & $\mathrm{Y}$ & 2 \\
\hline 110 & RRP 108/127 & FNAL & MQXFA03 & 214 & 22.40 & $\mathrm{Y}$ & 1 \\
\hline 111 & RRP 108/127 & FNAL & MQXFA03 & 227 & 22.18 & $\mathrm{Y}$ & 5 \\
\hline 202 & RRP 108/127 & BNL & MQXFA03 & 240 & 22.82 & $\mathrm{Y}$ & 0 \\
\hline 204 & RRP 108/127 & BNL & MQXFA03 & 224 & 22.11 & $\mathrm{Y}$ & 0 \\
\hline
\end{tabular}

Three short model structures were built at CERN, with one shipped to the US and used in the first short model MQXFS1. As to the protoype program in the US, 13 coils were tested in four assemblies. The second prototype MQXFAP2 had a severe non conformity in one of the Al shells (see section 3.11), and therefore coil data are not given here since they are not significant for this analysis. Two coils were completely manufactured by BNL, the others either by FNAL, or wound by FNAL and completed by BNL.

Considering both short model and prototype, out of the 30 tested coils, about $1 / 3$ reached nominal without quench, and another third with one quench. The details of the test, including the different assembly conditions are given in the next section.

A short recall on the naming convention:
- Short models are identified by MQXFS;

- US prototypes are MQXFAP1 and MQXFAP2, the first one being $4 \mathrm{~m}$ long and the second with the final length (both manufactured by LARP);

- US-AUP series magnet are MQXFA03-23;

- CERN prototypes are MQXFBP1, MQXFBP2;

- CERN series is MQXFB01 to MQXFB10.

We finally recall that, as explained at the end of section 2.2, all HL-LHC magnets are required to ensure operation at ultimate current with a plateau of $8 \mathrm{~h}$, corresponding to the expected duration of a physics fill. No engineering margin is added to the value of ultimate current. 


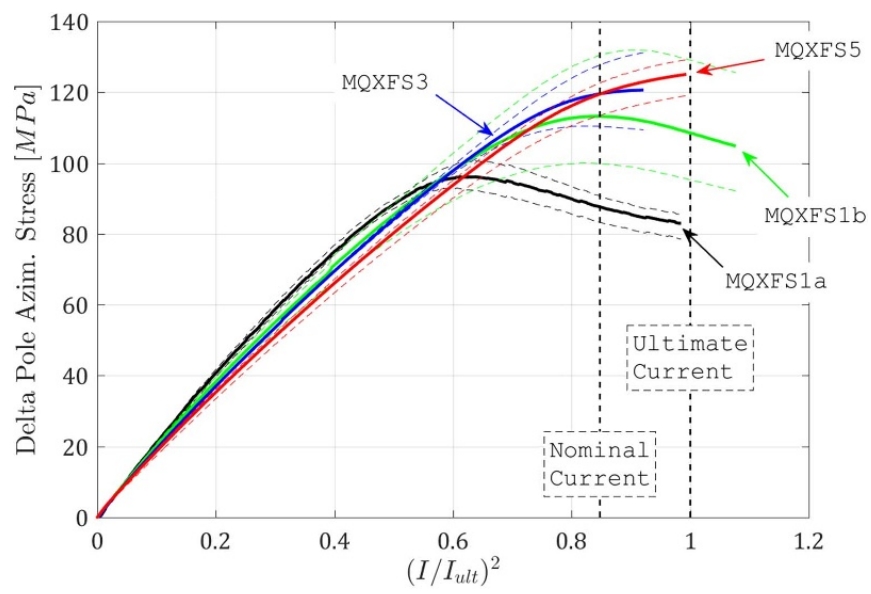

Figure 14. Pole unloading in three short models: measured stress variation at the pole versus square of the current, normalized to ultimate current.

The first short model MQXFS1 had two coils from CERN and two coils from LARP, made with RRP strand with different layouts (see table 2). The magnet was precompressed with $\sim 100 \mathrm{MPa}$ at $1.9 \mathrm{~K}$, i.e. to prevent the beginning of unloading up to $14 \mathrm{kA}$, i.e. $2 \mathrm{kA}$ lower than the nominal current, as confirmed by mechanical measurements (see figure 14, where the plateau in the stress-current ${ }^{2}$ curve is considered a sign of pole starting to unload). It reached nominal current (i.e. $7 \mathrm{TeV}$ operation, see section 2.1) with six quenches (see figure 15), and ultimate current (7.5 TeV operation) with another seven quenches. It reached the nominal current at $4.5 \mathrm{~K}$ and also after a thermal cycle without the need of additional training [91].

In a second assembly MQXFS1b, the precompression was increased to $120 \mathrm{MPa}$. This prevents unloading up to $\sim 16 \mathrm{kA}$, i.e. close to nominal current, as confirmed by mechanical measurements (see figure 15). The magnet reached ultimate current without retraining, but showed some setbacks around ultimate (see figure 15, around quench n. 40-45) [91].

A second iteration MQXFS1c on the magnet mechanics was done by increasing the axial precompression (see figure 16). The magnet reached nominal without quenches, and ultimate current with a slightly erratic behaviour in another four quenches. Finally, the magnet was used to test the assembly and welding of the stainless steel shell, included in the design as He containment, and showed similar behaviour to assemblies MQXFS1b and MQXFS1c. In total, the magnet underwent four thermal cycles and more than 100 quenches, always reaching nominal current without quench and, with few quenches, always reaching ultimate current [92]. This is the first short magnet to satisfy the performance requirements, showing a considerable operational margin and good properties in terms of memory (no retraining for nominal current).

The model MQXSF3, with three coils from CERN and one from LARP, was precompressed with $120 \mathrm{MPa}$, as MQXFS1b (see figure 17). The magnet reached nominal current with eight quenches, but after reaching $17 \mathrm{kA}$ it had a detraining in coil number 7 . The situation was unexpectedly recovered

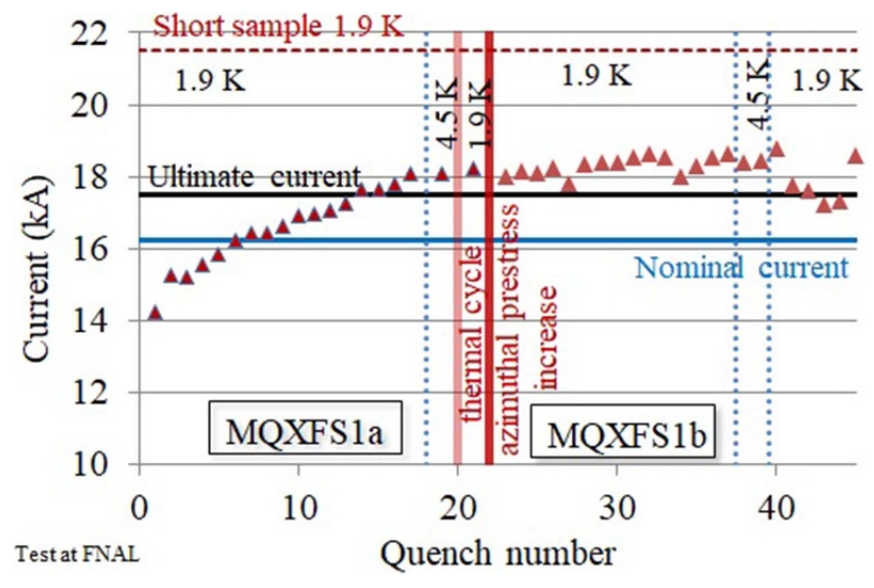

Figure 15. Training of the short model MQXFS1 (first part).

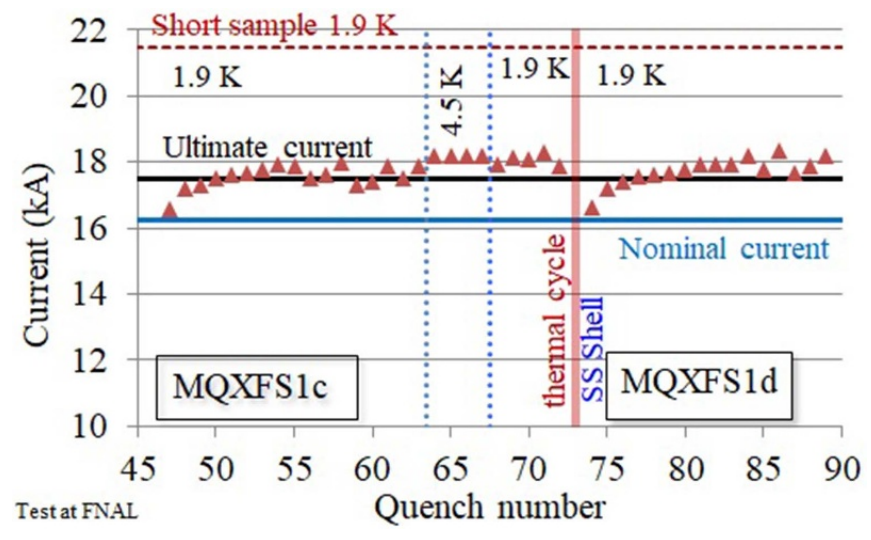

Figure 16. Training of the short model MQXFS1 (second part).

with a high ramp rate test, and $17 \mathrm{kA}$ current was reached at $4.5 \mathrm{~K}$. After a thermal cycle allowing to increase the axial prestress, the magnet was limited at $1.9 \mathrm{~K}$ just above nominal current (see figure 17, MQXFS3b), but reached $1.5 \mathrm{kA}$ more at $4.5 \mathrm{~K}[93,94]$. The limiting coil was replaced in assembly MQXFS3c, but another coil (number 105) was then blocking the magnet at $1.9 \mathrm{~K}$ well below nominal. This was a typical example of reverse behaviour, with the magnet better behaving at higher temperatures and higher ramp rates. Ultimate current was reached at $200 \mathrm{~A} \mathrm{~s}^{-1}$ ramp rate, and more than $17 \mathrm{kA}$ at 4.5 K. This suggests the existence of a complex mechanism of performance limitation due to conductor instabilities. At the moment of writing we have no justification of the limited performance of this short model.

The short model MQXFS5 was manufactured with a PIT conductor at CERN. It was precompressed with $140 \mathrm{MPa}$., i.e. full precompression for ultimate current. Strain measurements confirmed no unloading at ultimate current (see figure 14). The magnet reached nominal current with three quenches (see figure 18), and ultimate current with 18 additional quenches. Training was long but without any detraining. The magnet showed perfect memory at ultimate current, and a quench level at $4.5 \mathrm{~K}$ above ultimate current. This is the second short magnet, after MQXFS1, to satisfy the performance requirements, showing a considerable operational margin 


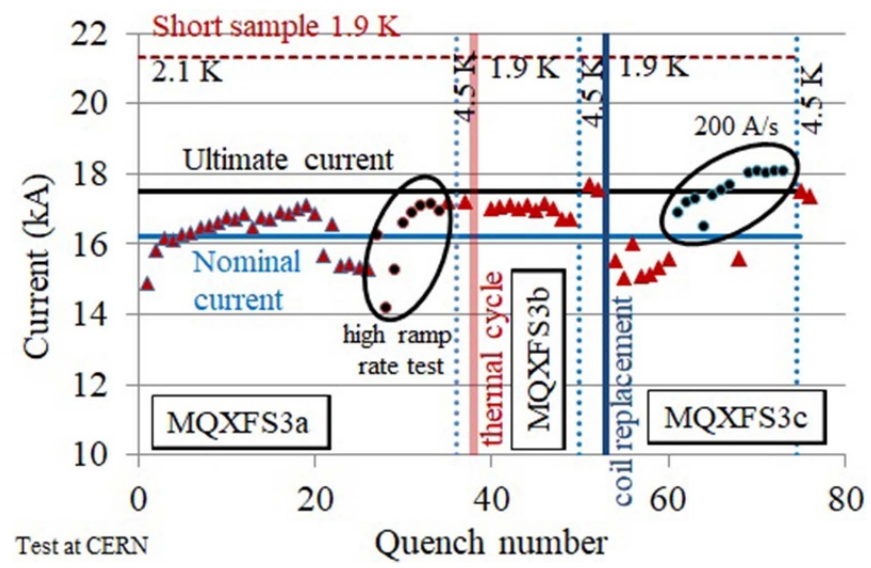

Figure 17. Training of the short model MQXFS3.

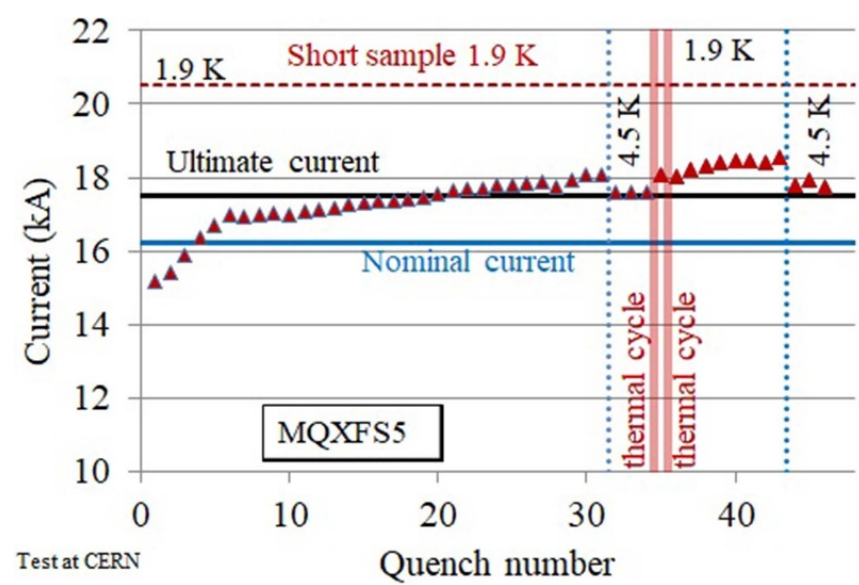

Figure 18. Training of the quadrupole short model MQXFS5.

and extremly good properties in terms of memory (no retraining to ultimate current).

The short model MQXFS4 was the first one with four coils made with 108/127 RRP strand, corresponding to the solution adopted for the series magnets both in the US and at CERN. Notwithstanding the numbering, it was assembled after MQXFS5. The coils were all manufactured at CERN. It was precompressed with $120 \mathrm{MPa}$, an intermediate level that has been adpoted for all future magnets of the project, guaranteeing coil compression up to nominal current. It reached nominal current without quench (see figure 19), and ultimate current with three quenches [94]. The magnet had perfect memory at ultimate current plus $500 \mathrm{~A}$, and reached ultimate current plus $500 \mathrm{~A}$ also at $4.5 \mathrm{~K}$. It went through an endurance test, with eight thermal cycles, showing no signs of degradation. The magnet has not yet been trained above $18 \mathrm{kA}$. This is the third short model to satisfy the performance requirements, after MQXFS1 and MQXFS5.

The second layout for PIT strand (with bundle) was used to manufacture four coils that were assembled in MQXFS6. Two coils had extremely low RRR values, well below the specificed values ( $75 \mathrm{vs}$ a minimum allowed of 100). One of them was shown to be the limiting coil in the magnet, that barely reached nominal current. In this case there was no indication of reverse

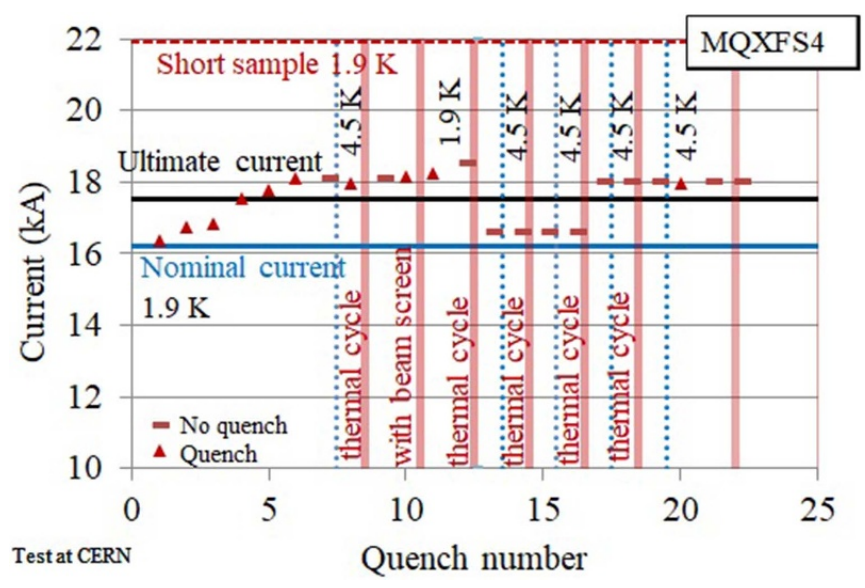

Figure 19. Training of the quadrupole short model MQXFS4.

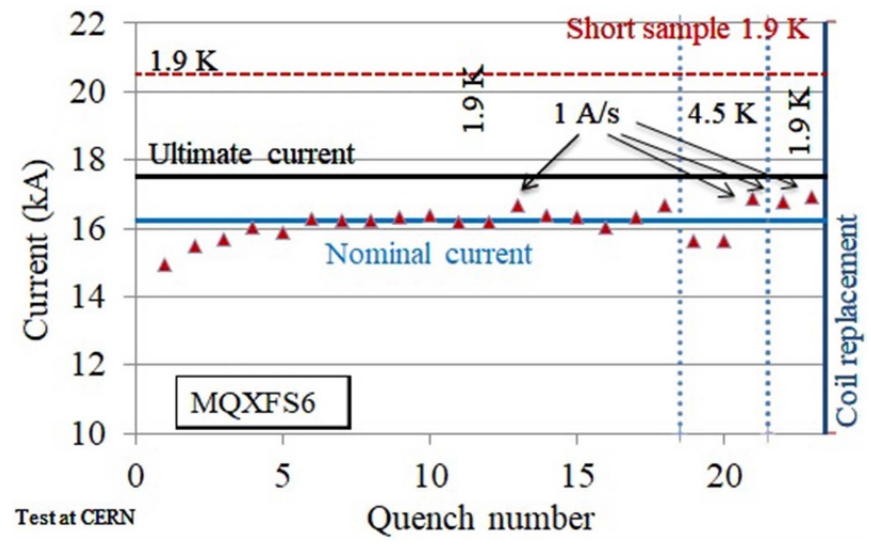

Figure 20. Training of the quadrupole short model MQXFS6.

behaviour, as the magnet had worse performance with higher ramp rates and higher temperatures (see figure 20). The missing performance of MQXFS6 was attributed to the very low RRR coils, that were replaced with two coils of MQXFS5. The new assembly MQXFS6b reached utimate without quench, and was trained up to a record of $19.14 \mathrm{kA}$, corresponding to $93 \%$ of the short sample (see figure 21). After the thermal cycle, the magnet quenched above $19 \mathrm{kA}$, i.e. above $90 \%$ of the short sample, showing a large potential of $\mathrm{Nb}_{3} \mathrm{Sn}$ technology. It also reached $98 \%$ of the short sample at $4.5 \mathrm{~K}$. This is the fourth short model magnet to satisfy the performance requirements.

After the $6 \mathrm{~b}$ test, the magnet was reassembled (6c) with a preload level as in MQXFS1 (low part of the assembly window). The magnet was able to operate at nominal current without any retraining, and reaching ultimate current with some training, thus confirming the wide range of preload allowing to reach the project requirements. On the other hand, the magnet was able to reach a maximum value of $18 \mathrm{kA}$, losing about $1 \mathrm{kA}$ in the performance with respect to previous assembly. This shows that a large preload is required to reach the range in $0.85-0.95$ loadline fraction. The magnet was finally assembled again in version $6 \mathrm{~d}$, with the original loading of $6 \mathrm{~b}$, and reached $19.5 \mathrm{kA}$ with a $13.4 \mathrm{~T}$ peak field. 


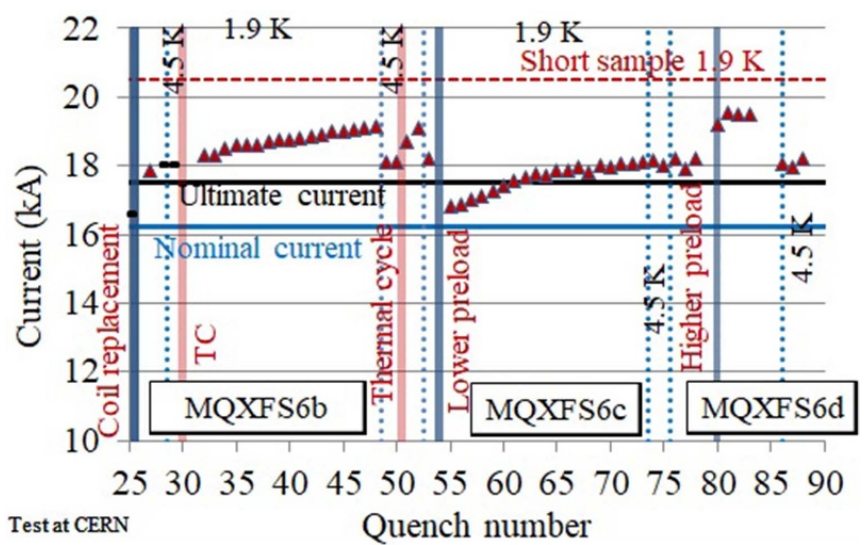

Figure 21. Training of the quadrupole short model MQXFS6b-d.

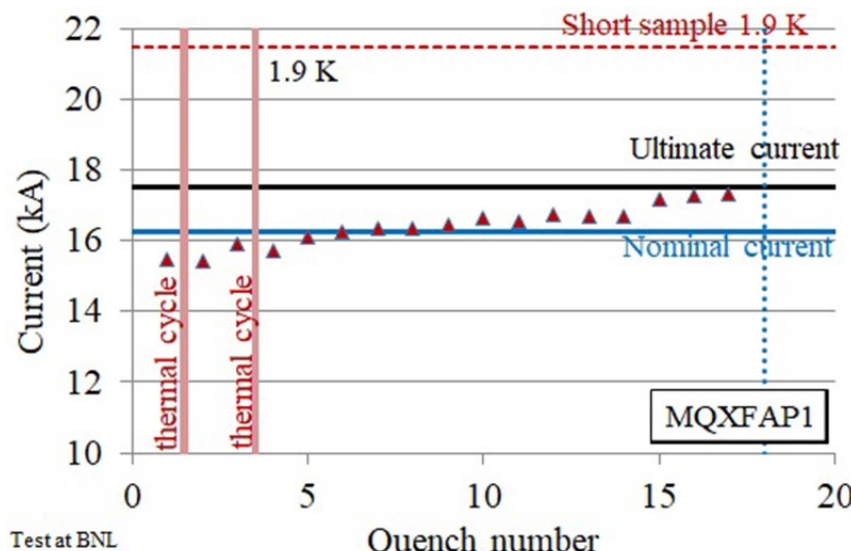

Figure 22. Training of the quadrupole prototype MQXFAP1.

The first prototype from the US-LARP had $4 \mathrm{~m}$ long coils, and RRP strand with different layouts (see table 2). The magnet was trained to nominal current with six quenches (see figure 22), and the test was stopped due to an electrical short, 200 A below ultimate current, after 17 quenches. This magnet proved the ability to reach nominal field on a $4 \mathrm{~m}$ long model [95].

Having replaced the faulty coil, the magnet was reassembled in the configuration called MQXFAP1b. This magnet performance (see figure 23) was limited by coil P03 previously tested in MQXFAP1 (see table 2). That coil had four consecutive quenches just around nominal in MQXFAP1, but then reached $17 \mathrm{kA}$ without quench. In the second assembly, the training to ultimate was fast but erratic, and finally the magnet reached only $13 \mathrm{kA}$ at $20 \mathrm{~A} \mathrm{~s}^{-1}$. Moreover, it quenched when the current was stopped on a plateau even below $12 \mathrm{kA}$. All quenches were in coil P03 [95]. As for the case of MQXFS3, at the moment of writing we have no justification of the erratic behaviour of this prototype.

The case of the second prototype MQXFAP2 is treated in section 3.13. The first US-AUP preseries magnet MQXFA03 reached nominal current with nine quenches, eight of which in the same coil. It was trained to $200 \mathrm{~A}$ above nominal current, and had no retraining after the thermal cycle (see figure 24).

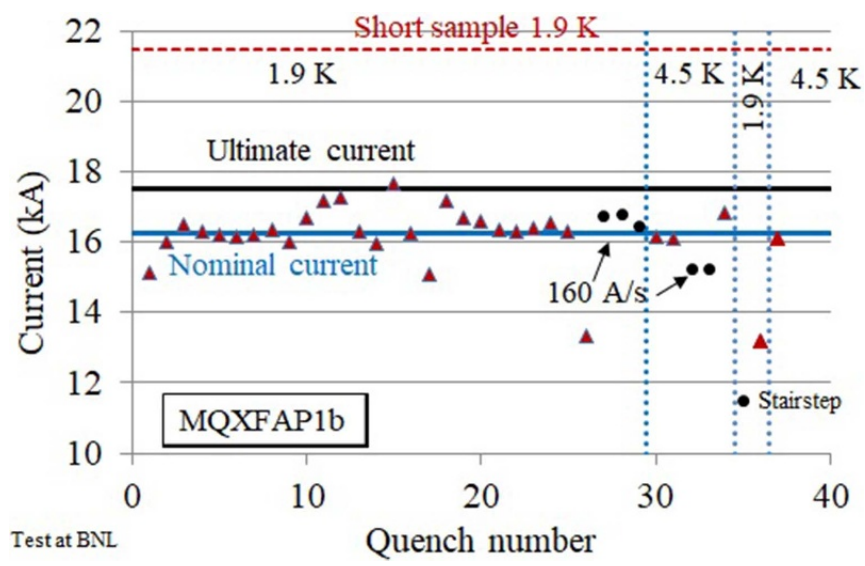

Figure 23. Training of the quadrupole prototype MQXFAP1b.

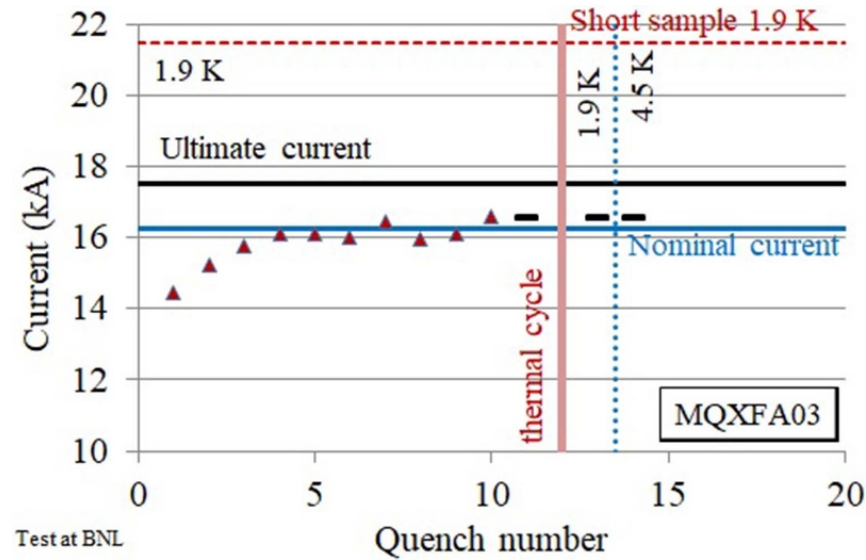

Figure 24. Training of the quadrupole MQXFA03.

This is the second full-size magnet to reach nominal current and the first one with a full validation of memory, showing no need of retraining for nominal current. The magnet shall be tested to ultimate current once assembled in a cold mass with another preseries magnet. This test is particularly important since it also validated the second coil production line at BNL.

\subsection{Design validation via power tests: field quality}

As previously stated, field quality is optimized at nominal current only. For the random part, the expected field errors are based on a random displacement from the nominal position with $30 \mu \mathrm{m}$ (one sigma) Gaussian distribution for the cable blocks; these displacements generate a distribution of multipoles used in beam dynamics simulations to confirm that particle stability is not affected. Therefore, specifications are given in terms of average and sigma, and the derivation of a tolerance band for acceptance is a non trivial passage. For the acceptance of the single magnet we set indicative tolerances at four sigma, as shown in figure 25 . Indeed, if systematic multipoles are at the edge of the band, fine tuning of the conductor layout are taken.

Fine tuning of field quality is guaranteed by two separate mechanisms for allowed and not allowed multipoles. Firstly, 


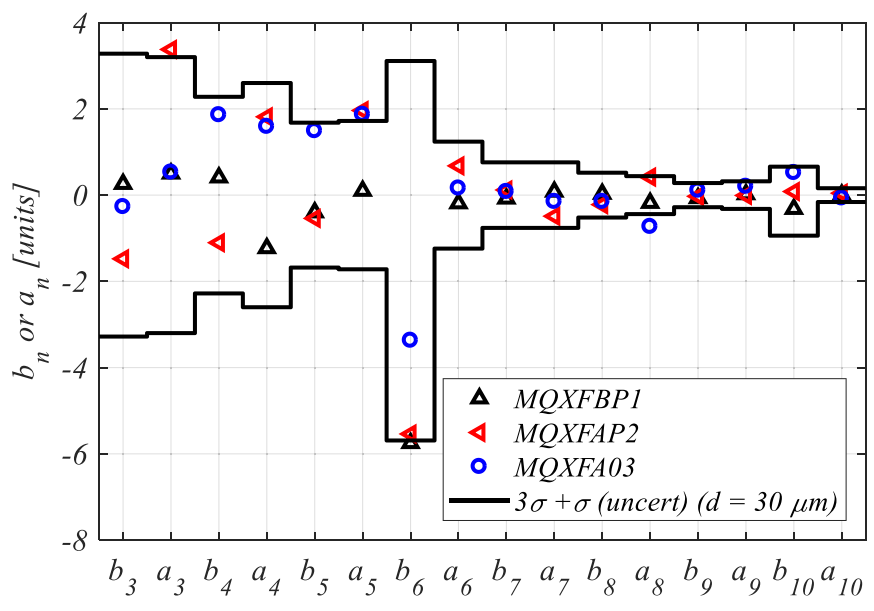

Figure 25. Measured multipoles versus $4 \sigma$ range for prototypes.

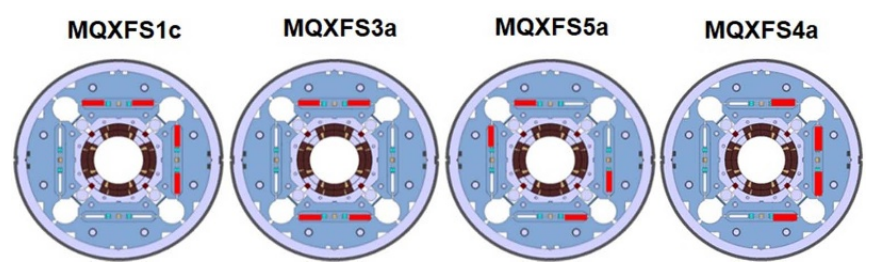

Figure 26. Magnetic shimming adopted on short models.

acting on the redundant insulation layer in the midplane and in the pole allows a fine tuning of the allowed multipoles. Moreover, magnetic shims can be inserted in the eight slots that are used for the bladders; they allow to correct 5 units of $b_{3}, a_{3}, 1$ unit of $b_{4}$ and 3 units of $a_{4}$. Two multipoles can be corrected at the same time. Following the standard conventions used for accelerator magnets, one unit is defined as $10^{-4}$ times the main component at the reference radius of $50 \mathrm{~mm}$ (one third of the aperture).

As shown in figure 25, the $b_{6}$ values are at the edge of the acceptance band [96]. The measurements on the first models showed a need of a $b_{6}$ correction of about +4 units to bring it around zero at the beginning of the production. Therefore, a $125 \mu \mathrm{m}$ shim was removed from the midplane and added to the pole, starting from MQXFA04 and MQXFBP2.

Some concern was present in the initial phase of the project for the non allowed multipoles. As already stated, the bladder and key structure provides a loading based on stress and not on displacement, therefore potentially allowing large non-allowed multipoles. The initial data on the short models confirmed large values of low order multipoles. The magnetic shimming strategy was succesfully tested to correct non allowed multipoles in all short models [96]. At the same time, the strength of the high order correctors was doubled to cope with larger errors (see also section 8.4). The data relative to a more mature part of the production revealed a much better level of field quality as shown in figure 25, namely the first results were due to the early phase of coil production and not to the assembly procedure. However, magnetic shimming has been tested on the short models to validate and acquire experience with the procedure. The four configurations tested
Table 3. Computed vs measured impact of magnetic shimming on short models.

\begin{tabular}{ll}
\hline & Computed \\
\hline MQXFS1c & $\Delta b_{3}=4.22$ \\
& $\Delta a_{3}=-4.24$ \\
MQXFS3a & $\Delta b_{4}=-2.88$ \\
MQXFS5a & $\Delta a_{4}=0.84$ \\
MQXFS3a & $\Delta b_{3}=3.42$ \\
\hline
\end{tabular}

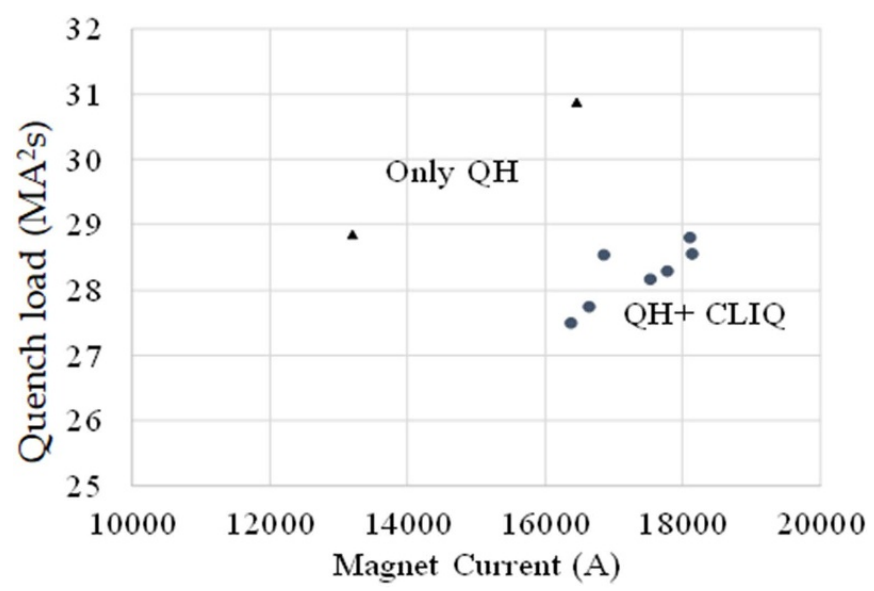

Figure 27. Quench load from quench detection vs current for different protection strategies.

are shown in figure 26, and the good agreement between the measured and expected correction of low order non allowed multipoles are given in table 3. Finally, one observes a very good correlation between the measurements after coil pack assembly, after loading, and at $1.9 \mathrm{~K}$. This proves that the coil pack already contains all the information about the final field quality, allowing to have a precious early indicator of any anomaly in field quality [96].

\subsection{Design validation via power tests: protection}

The protection strategy has been validated on short models without energy extraction [88], and on US prototypes with energy extraction [97]. It was found that, in agreement with simulations, the simultaneous use of CLIQ and quench heaters gives a quench load (integral of square of current over time, from quench detection) of 27-29 MIITs at current ranging between nominal and ultimate (see figure 27). This corresponds to hotspot temperatures of $260 \mathrm{~K}$ to $290 \mathrm{~K}$. In case of CLIQ failure, one has about 3.5 additional MIITs, corresponding to about additional $50 \mathrm{~K}$.

\subsection{Design changes}

Three main changes were carried out since the beginning of the project:

- The keystone angle of the cable was lowered from $0.55^{\circ}$ to $0.40^{\circ}$ to reduce the degradation of the PIT conductor during cabling. All coils of short model MQXFS1 and one coil 


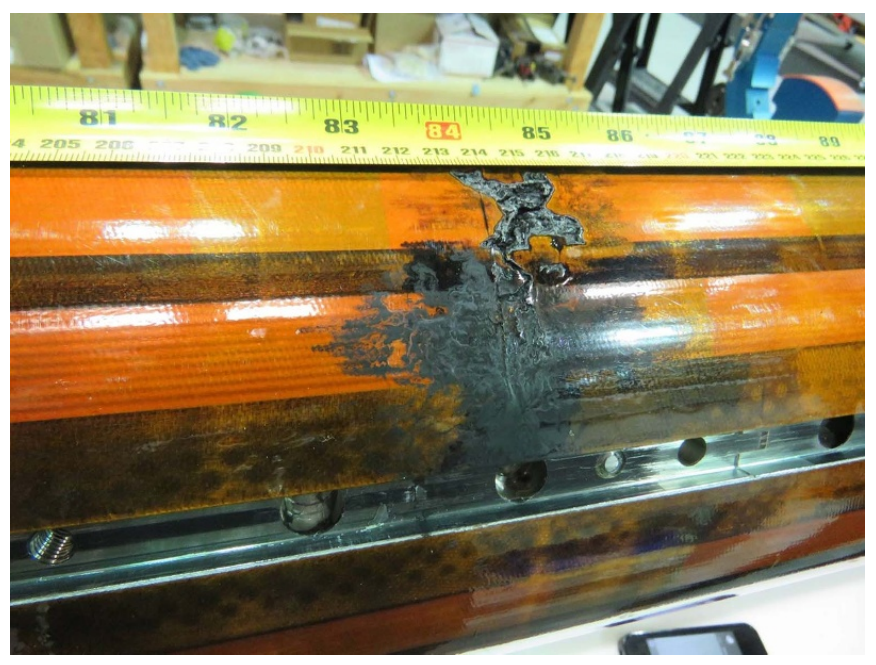

Figure 28. The short coil to heater in MQXFAP1.

of the first US prototype MQXFAP1 have this initial coil geometry [98].

- The magnet length has been increased by $5 \%$ to lower the loadline fraction from 0.82 to 0.77 . Therefore, the magnet length was increased from $4.0 \mathrm{~m}$ to $4.2 \mathrm{~m}$ for MQXFA, and from 6.8 to $7.15 \mathrm{~m}$ for the MQXFB, to lower the operational gradient from $140 \mathrm{~T} \mathrm{~m}^{-1}$ to $132.6 \mathrm{~T} \mathrm{~m}^{-1}$. At the time of the change, the first prototype coils in the US were already being manufactured and therefore the first US prototpye MQXFAP1 has $4.0 \mathrm{~m}$ long coils. All the other prototypes coils (CERN and US) have final length.

- A $125 \mu$ m-thick shim has been moved from the coil midplane to the pole to increase $b_{6}$ by 5 units, as described in the previous section.

\subsection{Setbacks and open issues}

The short model and prototype phases had three understood setbacks. As said in section 3.9, the first prototype MQXFAP1 had a double short between a coil and a quench heater, which allowed excessive current flowing through the heater during quench: as a consequence, one coil was lost (see figure 28). A cause of this incident was found in the poor quality impregnation of that coil, that was among the first prototype coils and used a non conform insulation fabric.

The second prototype MQXFAP2 had a non-conforming $\mathrm{Al}$ shell without fillet radius at some corners, provoking high stress concentration and eventually a complete breakage of the shell during test (see figure 29). Nevertheless, the magnet reached $14 \mathrm{kA}$ (see figure 30)—a remarkable value showing the resiliance of the mechanical structure. After this incident, the design of the cut-out in the Al shells was modified introducing larger radii.

The short model MQXFS6 had non conforming RRR in two coils ( 75 compared to the specification of $>100$ ); this is believed to be the reason for the limited performance of the magnet, barely reaching nominal current at $1.9 \mathrm{~K}$. Low RRR is one of the triggers of instabilities in the strand [77, 78]. On

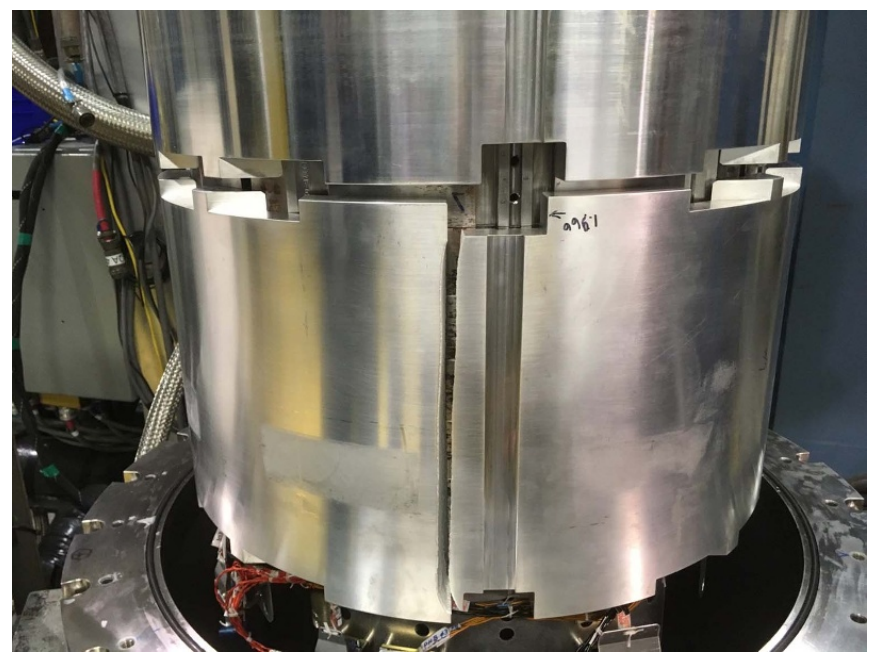

Figure 29. The broken shell in MQXFAP2.

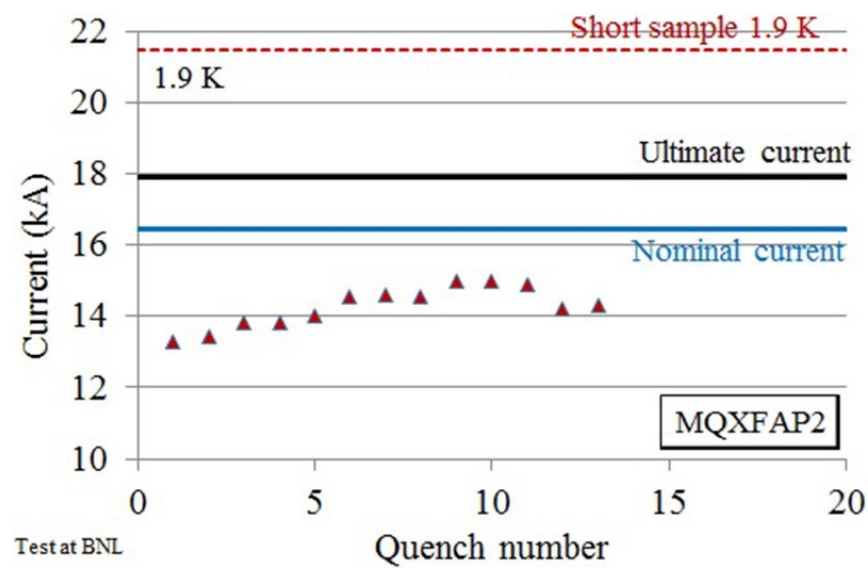

Figure 30. Training of the quadrupole MQXFAP2.

the other hand, it must be pointed out that we have coils with RRR ranging between 80 and 95 that reached performance.

As reported in section 3.10, we have two more cases of limitations in performance, with strong traces of reverse behaviour (MQXFS3 and MQXFAP1b) for which we have no explanation. This is the main open issue for MQXF at the moment of writing.

\subsection{Timeline and schedule}

The main milestones of the quadrupole development are the followings, including the steps under LARP.

- LARP TQ (90 mm aperture, $1 \mathrm{~m}$ long, collar stracture TQC and bladder and key TQS)

* 2005: start of design;

* 2007-2009: test of five short models;

- LARP LQ (90 mm aperture, $4 \mathrm{~m}$ long)

* 2007: start of design;

* 2010-2012: test of three LQ;

- LARP HQ (120 mm aperture, $1 \mathrm{~m}$ long)

* 2007: start of design; 
* 2010-2012: test of three HQ;

- October 2011: beginning of the HL-LHC design study;

- July 2013: selection of triplet aperture of $150 \mathrm{~mm}$;

- MQXF short model

- July 2013: start of design;

* March 2016-March 2019: test of five short models

- MQXF full size

* March 2015: beginning of long coil manufacturing by FNAL and BNL;

* March 2016: beginning of long coil manufacturing by CERN;

* August 2017-February 2018: test of the first prototype MQXFAP1;

* August 2019: test of the second prototype MQXFAP2;

* December 2019: test of the first preseries magnet MQXFA03.

Given the previous experience of LARP, and the preparatory work in the HL-LHC design study, the time from the aperture selection to the test of the first short model has been less than 3 years. The synergy between the laboratories has also been profitful for the prototypes: CERN making most of the short model development, AUP has been able to test a full size prototype 4 years after the aperture selection.

US-AUP shall build 20 magnets, plus the first magnets built within LARP, and CERN shall build 12 magnets. At CERN, the production line of one winding machine, one reaction oven and one impregnation system can produce one coil in about 5 months, with a maximum rate of one coil per 3 weeks. Magnet assembly is done in 3 months, giving a total of 11 months for manufacturing one magnet. Accounting for vacations, tooling maintenance, and five coils per magnet, one can reach a rate of three magnets per year. A similar rate is considered in the US, where 4.5 coils per magnet are assumed, and two production lines are operational: one at FNAL and one at BNL. Coil manufacturing is driving the magnet schedule production rate.

\section{The separation dipole}

\subsection{Accelerator requirements}

The separation dipole D1 is a single aperture magnet with $150 \mathrm{~mm}$-diameter bore and $35 \mathrm{~T} \mathrm{~m}$ nominal integrated field. The magnet function is to increase the distance of the counterrotating beams from zero (as it is in the experiments) to $192 \mathrm{~mm}$ (as it is in the LHC arcs), over the $\sim 65 \mathrm{~m}$ distance between D1 and D2. The magnet is individually powered and ramps proportionally to the LHC energy from $450 \mathrm{GeV}$ to $7 \mathrm{TeV}$. Field quality requirements are set at $7 \mathrm{TeV}$ energy, with all multipoles at reference radius of $50 \mathrm{~mm}$ below 1 unit with the expection of $b_{3}$, for which a larger tolerance of 3 units is accepted. No requirements are given on the saturation of the main dipolar component that can be compensated via the power converter. No requirements are given on the field quality at injection as for all the IR magnets. The most exposed part of the magnet have to resist to 15 MGy dose over the HL-LHC lifetime.

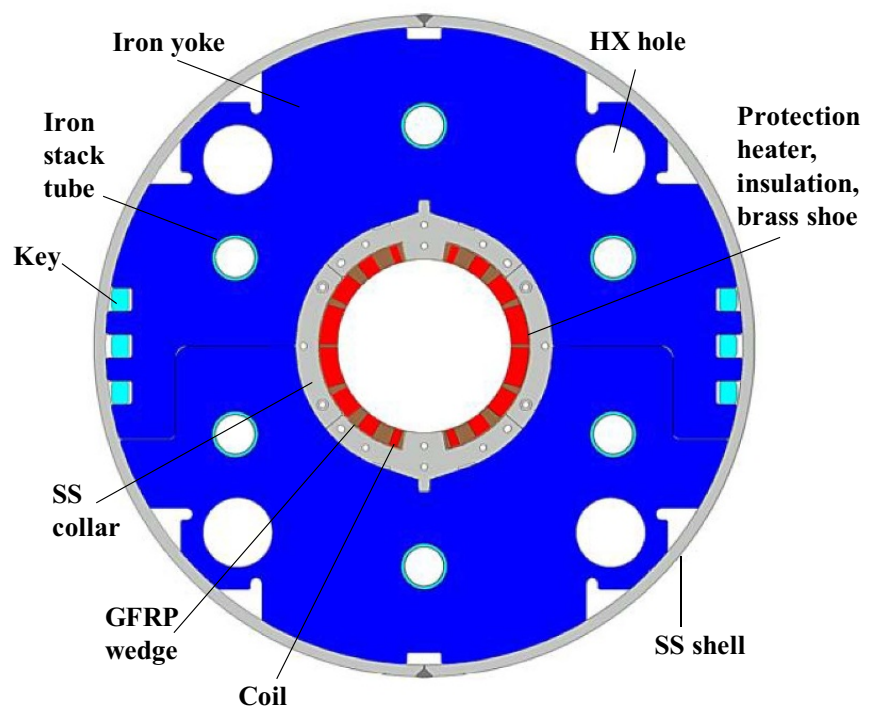

Figure 31. Cross-section of the separation dipole.

\subsection{Design features}

The integrated field is realized via a $5.6 \mathrm{~T}$ nominal field over a $6.2 \mathrm{~m}$ magnetic length [44], produced by a $15 \mathrm{~mm}$-width $\mathrm{Nb}-\mathrm{Ti}$ coil that reuses the cable of the main LHC dipole, outer layer (see figure 31). We refer to the existing literature for the properties of the strand, of the cable and of the insulation. As previously stated, at nominal current the magnet operates at a loadline fraction of 0.77 .

The coil has four blocks: three would have been enough to satifsfy the field quality requirements, but four blocks give a larger flexibilty to make fine tuning of the multipoles. There are 19 turns in the first block, 13 in the second, 8 in the third and 4 in the fourth. As in the RHIC dipole design [99] and in LHC insertion region magnet MQXA quadrupole design [18], the mechanical structure is based on iron yoke laminations with three keys on each side. Ten millimetres-thin spacers are used to place the iron as close as possible to the coil, maximizing the its contribution to the main field. Contrary to RHIC dipole, the spacers are not in fiberglass but in stainless steel; they are called collars even though they are not active part of the mechanical structure.

The main challenge of this magnet is the large accumulation of stress in the midplane due to electromagnetic forces. As quoted in section 2, one has $100 \mathrm{MPa}$ in the midplane, that is about twice the value of the LHC main dipole and approximately the same as in the $\mathrm{Nb}_{3} \mathrm{Sn}$ HL-LHC magnets. This is an unprecedented value for $\mathrm{Nb}-\mathrm{Ti}$ accelerator dipoles.

The second main challenge of the magnet is achieving the field quality target. The saturation has a strong effect not only on the main component, but also on the multipoles [45, 46]. Thanks to the iron shape optimization, the variation of $b_{3}$ during the ramp is reduced to 20 units; however, coil geometry is set to minimize $b_{3}$ at nominal current.

An additional issue is that for the $2 \mathrm{~m}$ long model the ratio between length and aperture is such that a $3 \mathrm{D}$ computation of the full magnet is needed even for the field quality modeling 


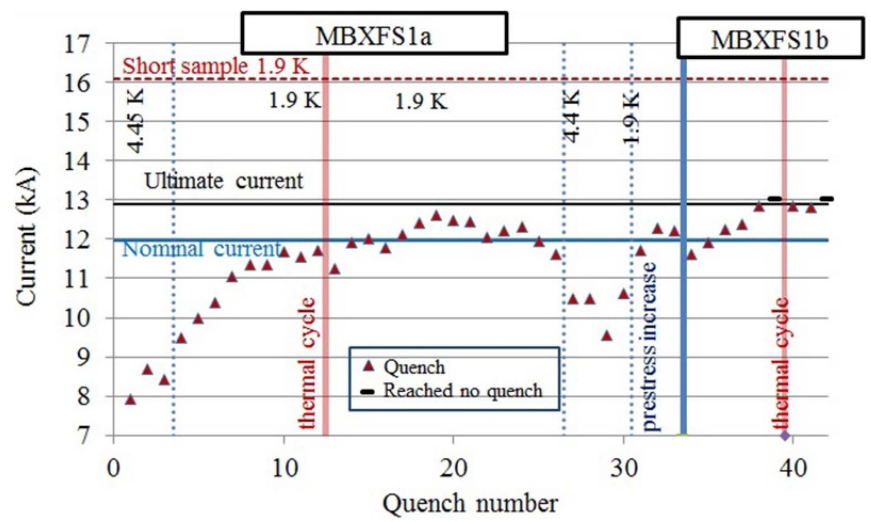

Figure 32. Training of the first separation dipole short model.

in the straight part $[48,49]$. So field quality extrapolation from $2 \mathrm{~m}$ long models to $6 \mathrm{~m}$ long prototypes is not straightforward and must be done with proper numerical tools.

The protection is obtained by the same technology as in the LHC main magnets, i.e. quench heaters on the outer radius of the coil. A maximum hotspot temperature of $300 \mathrm{~K}$ is set as a limit, including the case of two heater failures [50, 51]. The energy extraction option was discared in the initial phase of the design for cost reasons.

\subsection{Design validation via power tests}

KEK planned for manufacturing two short models, one prototype, four series and two spare magnets. Prior to the short model construction, a mechanical model was done to validate the coil size, the shimming to reach nominal precompression, the yoking and assembly procedures.

The first short model MBXFS1 reached nominal current after 15 quenches [46], and had erratic behaviour between nominal and ultimate current (see figure 32). The short model azimuthal coil size turned out to be much smaller than what needed for full preload at ultimate current. This induced a loss of preload already at current values between 6 and $8 \mathrm{kA}$ (see figure 33). The coil had also an insufficient support of the ends, and after the first test a movement up to $4 \mathrm{~mm}$ in the coil turns towards the magnet aperture was observed in the coil heads.

The second assembly MBXFS1b included a $0.8 \mathrm{~mm}$-thick shim in the midplane to increase the precompression. The magnet reached performance: nominal current was reached after two quenches, and ultimate current after five quenches (see figure 32, after the vertical blue line). After thermal cycle no retraining was needed for nominal current, and two quenches for ultimate current [47]. The magnet showed pole unloading in the straight part above $12 \mathrm{kA}$, but was able to train up to $13 \mathrm{kA}$ (see figures 32 and 33).

In the second short model [48], wedges were enlarged by a total of $1.15 \mathrm{~mm}$ to have a larger azimuthal coil size aiming at full precompression at ultimate current. Moreover, there was an iteration on the iron yoke (see next section) and consequently on the coil cross section. The magnet reached performance (see figure 34): nominal current was reached after seven quenches, and ultimate current after 12 quenches. After

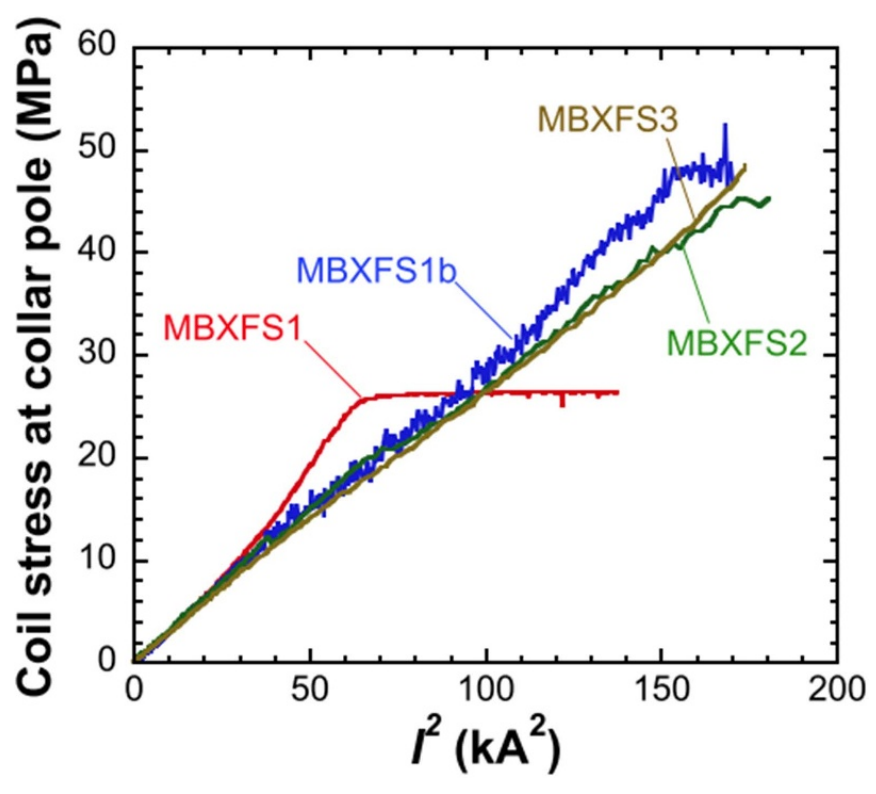

Figure 33. Strain gauge measurement of pole unloading during the MBXFS1, MBXFS1b, MBXFS2 and MBXFS3 powering.

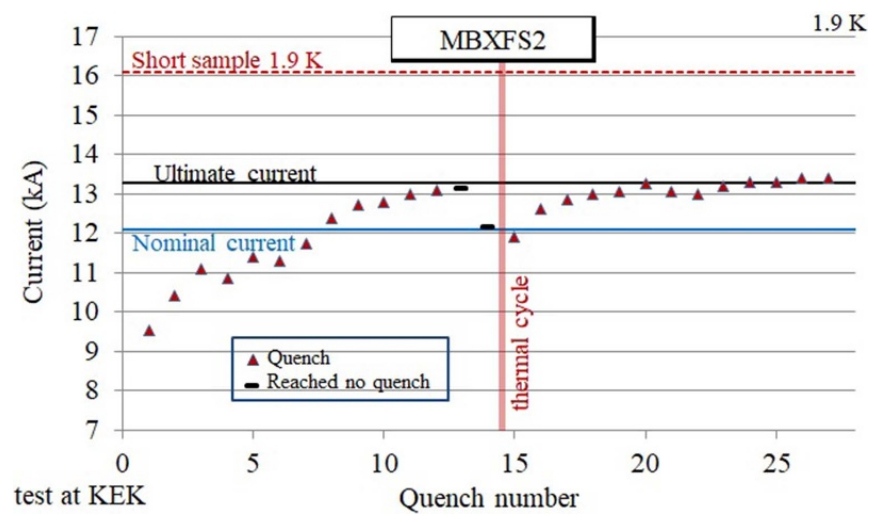

Figure 34. Training of the second separation dipole short model.

thermal cycle, one quench was required for nominal and about ten for ultimate. Strain gauges measurements confirmed that this magnet had a sufficient precompression in the straight part to avoid coil unloading at ultimate current, as planned (see figure 33).

After the second short model results, it was proposed to manufacture an additional short model to validate the performance and field quality reproducibility. The third short model, manufactured as a perfect copy of the second one, reached nominal current with one quench, and ultimate current with 20 quenches (see figure 35). It was tested at $4.4 \mathrm{~K}$, showing the ability of operating above nominal and therefore a temperature margin at nominal current above $2.5 \mathrm{~K}$. Training memory proved to be very good, with no quenches to nominal after thermal cycle and three quenches to ultimate. Strain gauges showed also in this case full precompression up to ultimate current (see figure 33). These results validated the design, allowing to start the construction of the prototype, that is ongoing in Hitachi at the time of writing (see figure 36). The 


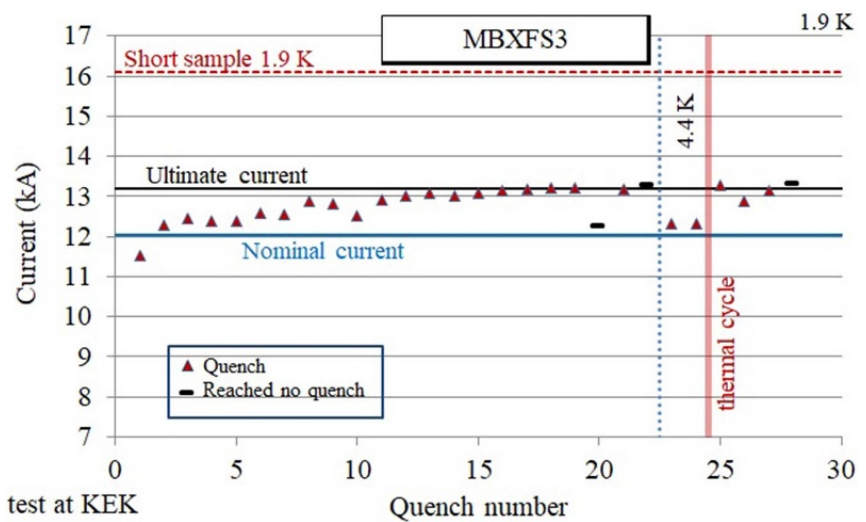

Figure 35. Training of the third separation dipole short model.

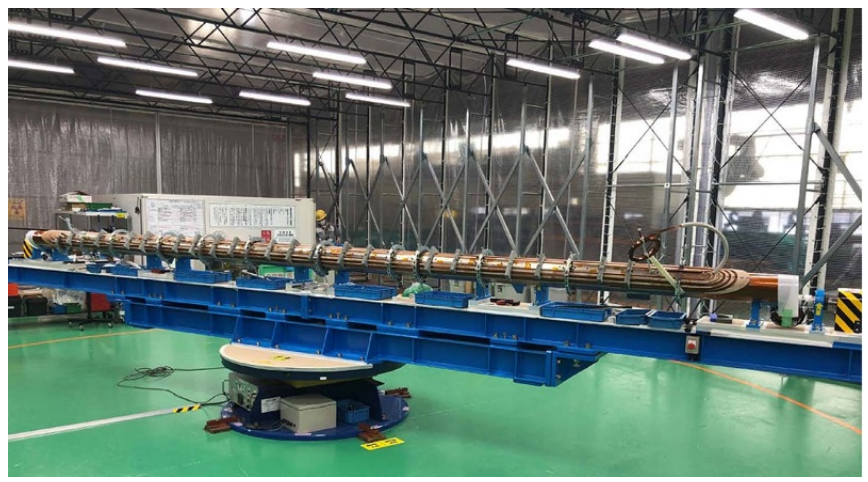

Figure 36. Winding of the coil of the D1 prototype.

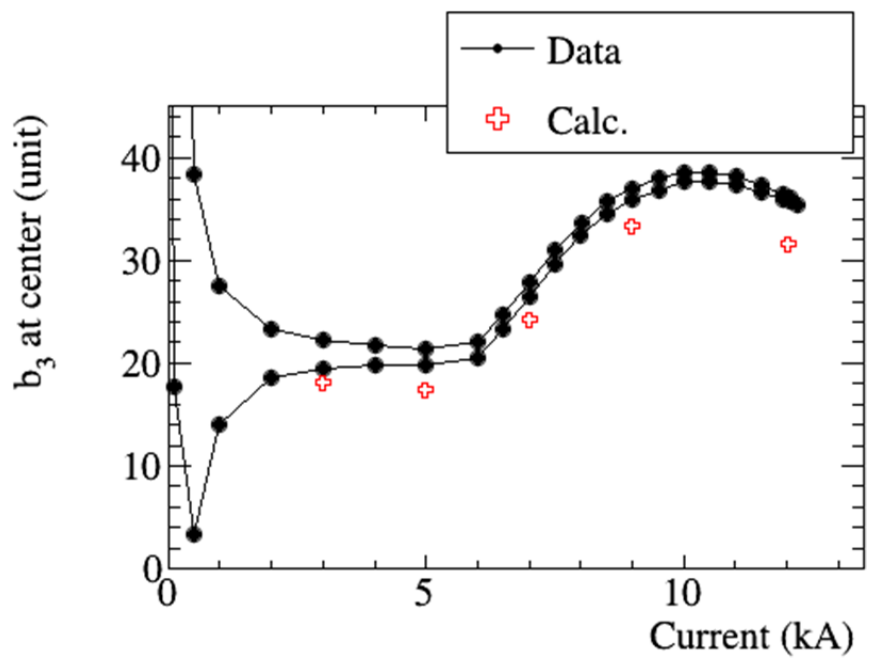

Figure 37. Measured versus modeled $b_{3}$ along the ramp in the second short model.

measured field quality agrees with expectations. The measurements of $b_{3}$ along the ascending and descending ramp, versus the OPERA model are shown in figure 37 for the short model MBXFS2.

\subsection{Design changes}

The design went through the following iterations [51].

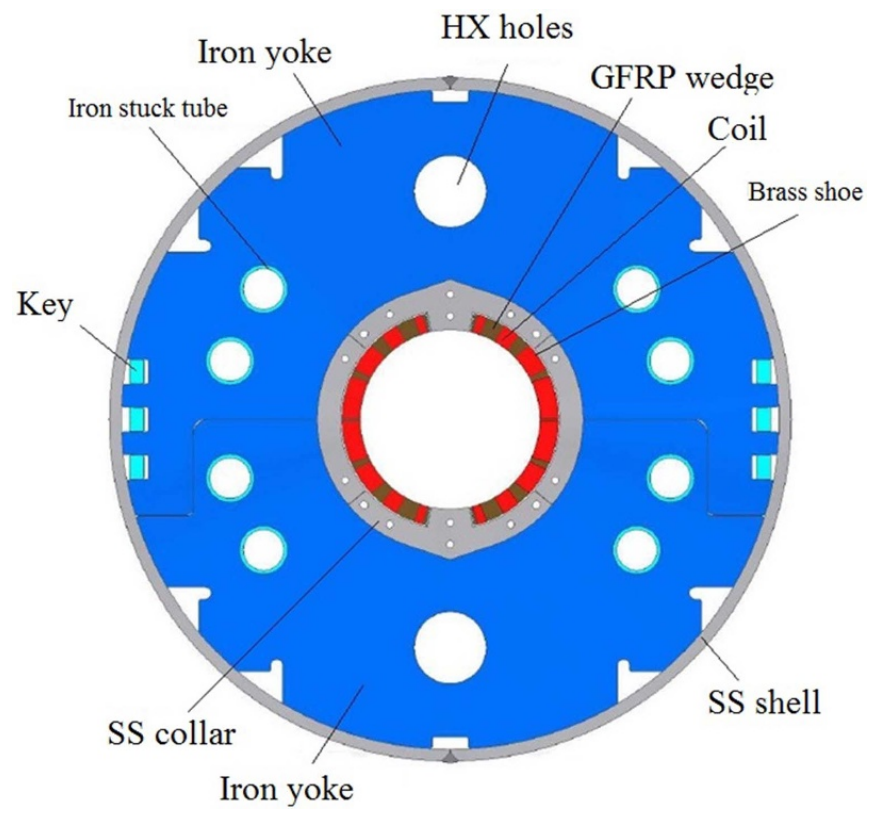

Figure 38. First cross-section of the separation dipole.

- After the mechanical model, the shape of the collar spacers was changed to improve the alignment of the assembly (see the change from the triangular shape of the collar in figure 38 to the alignment notch in figure 31 ).

- The position of the cooling holes was initially set to $90^{\circ}$ and $270^{\circ}$ (see figure 38 ); it was later moved to $45^{\circ}, 135^{\circ}$, $225^{\circ}$ and $315^{\circ}$ (as in the triplet, see figure 31 ) to account for the constraints due to interconnections.

- There has been a change of cross-section from MBXFS1 to MBXFS2 to better optimize field quality, both for the correction of the $3 \mathrm{D}$ effects coupled with saturation, that were ignored in the first layout, and for taking into account of the new geometry of the iron holes.

- The nominal magnetic field was increased by $2 \%$ to reduce the total length of the magnet below $6.5 \mathrm{~m}$, thus allowing vertical test in KEK. Without this reduction of length the magnet would have not fit the test station and the cost for an upgrade would have been not acceptable for the project.

- The quench heaters were initially a simple strip covering one coil block; this design proved to be not enough efficient to quench the coil. It has been replaced by two strips, zigzaging between three blocks of the magnet, and with copper coating in the transition between the blocks to reduce the total resistance of the strip. With this design, at nominal current the coil is quenched within $20 \mathrm{~ms}$.

\subsection{Setbacks and open issues}

The most relevant issue in this magnet is the control of azimuthal prestress in the straight part and in the coil ends. We had one setback in the first short model performance. The origin is clearly due to precompression in the coil, but it was not possible to state if the cause was the lack of prestress in the 


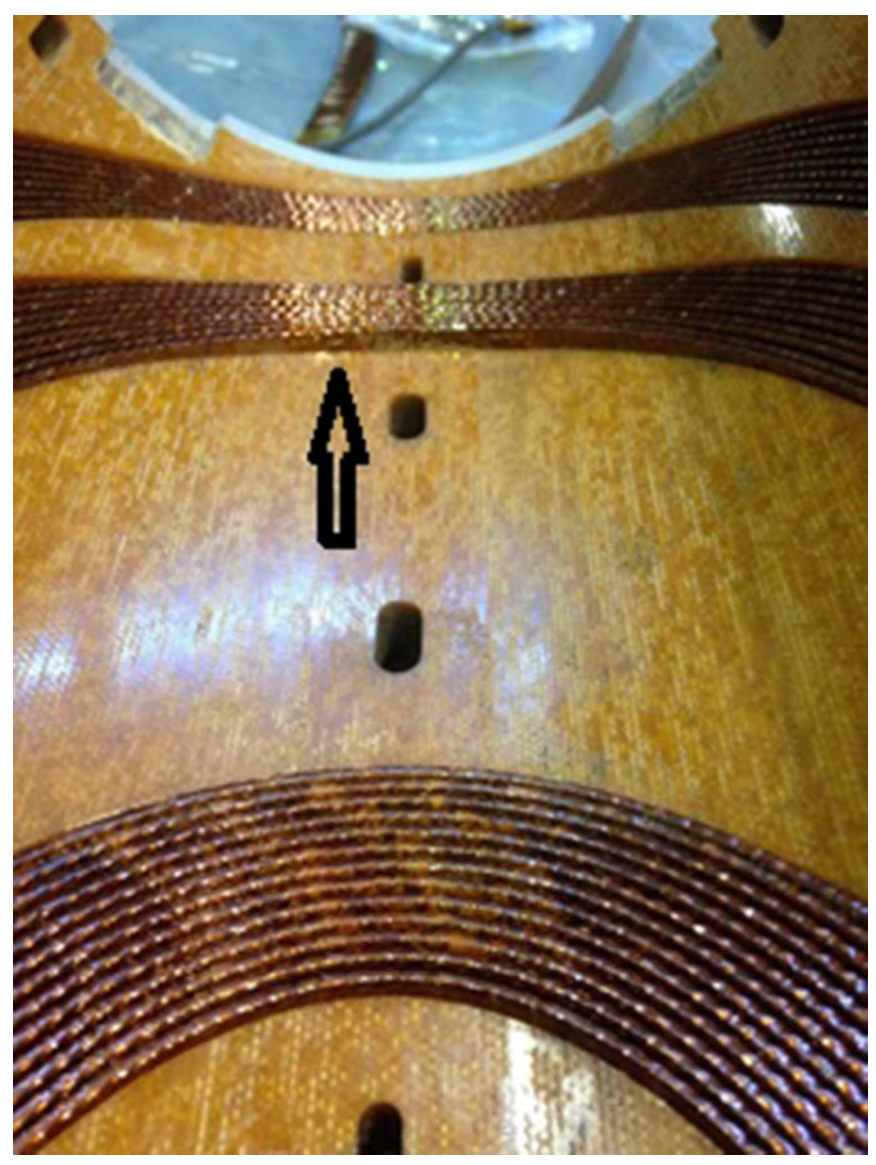

Figure 39. Cable protuding inside the aperture in the coil heads of MBXFS1 (see black arrow).

straight part or the lack of support in the coil end. As stated in the previous paragraph, movements of up to $4 \mathrm{~mm}$ of the coil in the ends towards the magnet aperture were observed in MBXFS1 after powering (see figure 39).

Both the second and third short model showed similar movements, but with much smaller amplitude (less than $1 \mathrm{~mm}$ ). This seems not to limit the performance, but is a source of concern for the series magnets. On the other hand, MBXFS2 data show that a partial unload around nominal current in the straight part does not prevent reaching ultimate current.

The other challenge of this magnet is the control of field quality, and mainly the low order harmonics at nominal current. The second and third model have an integral $b_{3}$ of about 40 units (see figure 36). Half of them are expected to disappear in the prototype, due to the dilution of end effects and to the reduction of saturation coupling with coil ends. The other half, whose origin is well understood, shall be corrected with a fine tuning of the wedges in the prototype. One should finally land on the \pm 3 units target allowed by beam dynamics. The way is long, but an additional iteration could be done (if needed) between the prototype and the series.

\subsection{Timeline and schedule}

The main milestones of the D1 development are the followings:
- October 2011: beginning of the design study;

- July 2013: selection of bore aperture;

- April 2014: beginning of coil manufacturing of the short model (practice coil);

- Mid 2015: mechanical model and iteration on the collars shape;

- April 2016: test of the first short model;

- February 2017: test of the second assembly of the first short model;

- October 2018: test of the second short model;

- May 2019: contract for prototype and series;

- September 2019: test of the third short model;

- April 2020: beginning of prototype winding.

The prototype and the six series magnets shall be built at Hitachi with an industrial contract steered and financed by KEK. The schedule is driven by the funding profile, with a rate of two magnets per year.

\section{The recombination dipole}

\subsection{Accelerator requirements}

The separation dipole D2 is a double-aperture dipole with $105 \mathrm{~mm}$-diameter bore and a $35 \mathrm{~T} \mathrm{~m}$ nominal integrated field. The magnet function is to decrease the distance of the counterrotating beams from $192 \mathrm{~mm}$ (as it is in the LHC arcs) to zero (as it is in D1), over the $\sim 65 \mathrm{~m}$ distance between D1 and D2. The two apertures are powered in series, with fields in the same vertical direction. The magnet is individually powered and ramps proportionally to the LHC energy from $450 \mathrm{GeV}$ to $7 \mathrm{TeV}$. The field quality requirements are set at $7 \mathrm{TeV}$ energy, with all multipoles at reference radius of $35 \mathrm{~mm}$ below 1 unit with the exception of $b_{3}$, for which a larger tolerance of 3 units is accepted. No requirements are given on the saturation of the main dipolar component that can be compensated via the power converter. No requirements are given on the field quality at injection as for all the IR magnets. The most exposed part of the magnet have to resist to $15 \mathrm{MGy}$ dose over the HL-LHC lifetime.

\subsection{Design features}

The integrated field is realized via a $4.5 \mathrm{~T}$ nominal field over a $7.8 \mathrm{~m}$ magnetic length, produced by a $15 \mathrm{~mm}$-width $\mathrm{Nb}-\mathrm{Ti}$ coil that reuses the cable of the main LHC dipole, outer layer, as in D1 (see figure 40). We refer to the existing literature for the properties of strand and cable. Cable insulation is made with two layers of $37.5 \mu \mathrm{m}$ thick polyimide.

This design [52-54] was also selected since INFN Genova and Milano had acquired experience with the FAIR SIS-300 fast-ramped dipole construction [100]. This dipole has very similar parameters, namely a $4.5 \mathrm{~T}$ field, a $100 \mathrm{~mm}$ aperture, and a one layer $\mathrm{Nb}-\mathrm{Ti}$ coil with the same LHC dipole outer cable. The main difference is that SIS-300 dipole is slightly curved, and it makes use of a strand with finer filaments $(3 \mu \mathrm{m}$ instead of $6 / 7 \mu \mathrm{m}$ as in the $\mathrm{LHC}$ ) to minimize the losses during fast ramp (up to $1 \mathrm{~T} \mathrm{~s}^{-1}$ ). 


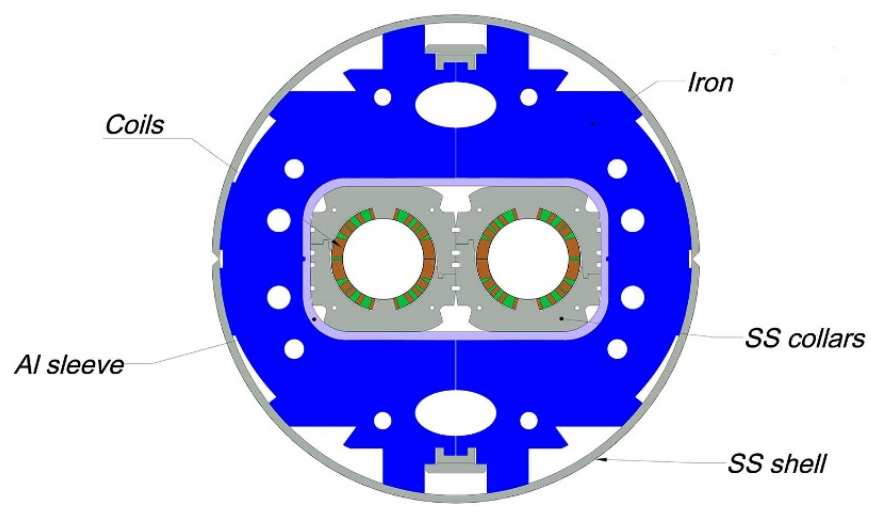

Figure 40. Cross-section of the recombination dipole D2.

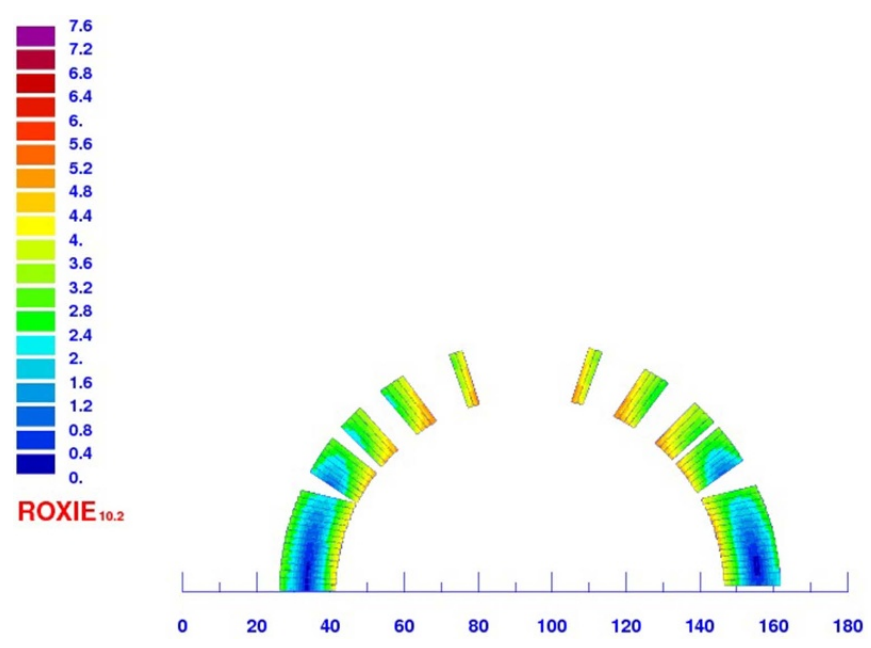

Figure 41. Cross-section of the coil of the recombination dipole D2.

In $\mathrm{D} 2$ recombination dipole, the main additional challenge with respect to SIS 300 is the double aperture, giving a nonnegligible magnetic cross-talk between apertures [52]. To be more quantitative, the $b_{2}$ component at $35 \mathrm{~mm}$ reference radius is of the order of $2 \%$ of the dipolar field (200 units). To reduce this cross-talk, the coils are left-right asymmetric (see figure 41) as proposed for the D2 dipole in [56].

The iron is far away from the coil, allowing self- supporting stainless steel collars. The limit in the bore field is set by the targets on the allowed multipoles; above $4.5 \mathrm{~T}$ the dependence of $b_{3}$ on current becomes very steep, due to iron saturation, and therefore it becomes very difficult to control. Note that with respect to D1, having the same cable and a similar current, $\sim 1 \mathrm{~T}$ is lost due to the cross-talk of the apertures (contrary to LHC dipoles, fields point in the same direction) and due to the lower impact of the iron. Last but not least, and differently to the LHC dipoles, independent collared apertures were selected. This allows larger flexibility for such a small production, and an easier collaring procedure, with a moderate cost increase. With these design choices, the magnet operates at a loadline fraction of 0.68 .

The coil has five blocks (see figure 41). Three blocks would have been enough to satifsfy the field quality requirements, and four were considered to be necessary to have enough free parameters to steer field quality, as for the D1. A main challenge was to find an asymmetric coil with the same number of turns per block on the right and on the left part, to avoid complexity in the coil heads. This challenging optimization problem was solved with five blocks, and was adopted for the short model $[52,53]$. Later, a four block solution was found with a clever optimization algorithm, but the model was already engineered and the redundant wedge was deemed to provide an additional free parameter for fine tuning of field quality, with a negligible extra cost.

There are 15 turns in the first block, six in the second, four in the third, four in the fourth and two in the fifth. Collars have a $25 \mathrm{~mm}$ thickness. The level of stress accumulation in the midlpane is $60 \mathrm{MPa}$, i.e. similar to the LHC dipoles (see figure 5): it is a challenging value in terms of precompression, but not at the level of the D1 previously discussed.

A novel solution is used to manage to repulsive force between the apertures: an $\mathrm{Al}$ sleeve is assembled at room temperature around the two apertures, with a $0.1 \mathrm{~mm}$ radial gap, and thanks to the larger thermal contraction it locks and aligns the two apertures at $1.9 \mathrm{~K}$. The sleeves, $10 \mathrm{~mm}$ thick, are warmed up during the assembly, and after the test were easily removed to test a second assembly

As in D1, the main challenge of the magnet is achieving the field quality target. All the optimization relies on the compensation of the two apertures and on the impact of the iron. Just to give the order of magnitude of the problem, the single aperture has 170 units $b_{3}$; when the two apertures are put together, $b_{3}$ moves to 70 units. When the iron is added, $b_{3}$ finally falls on the \pm 3 units range. So a compensation better than $95 \%$ is required. The field quality optimization relies on this delicate balance between coil cross-talk and iron shape. The good side is that these effects can be measured at room temperature. Moreover, the iron saturation is not so dramatic as in D1, as the field is $1 \mathrm{~T}$ smaller and the iron is $15 \mathrm{~mm}$ more distant from the coils.

The protection is guaranteed by the same technologies as in the LHC main magnets, i.e. quench heaters on the outer radius of the coil. A maximum hotspot temperature of $300 \mathrm{~K}$ is set as a limit, including the case of two heater failures. The energy extraction option was discared in the initial phase of the design for reasons of cost. The heaters cover three out of the five coil blocks, allowing to quench the magnet within 10-20 ms from quench detection at nominal current.

\subsection{Design validation via power tests}

INFN-Genova, in charge of the design, engineering and construction of the model, assigned the tender for the magnet manufacturing to ASG Superconductors (Italy). The program includes one double-aperture short model, one prototype, four series magnets and two spares. The short model test showed that the magnet was limited in one coil of one aperture at $10 \mathrm{kA}$ [55], i.e. about $2 \mathrm{kA}$ lower than nominal current (see figure 42). After disconnecting the faulty aperture, the other one reached nominal current without quenches, and ultimate current with two quenches. Note that for this single aperture test-not in the baseline - a fine tuning of the nominal/ultimate current concept was done to have the same loadline fraction as the 


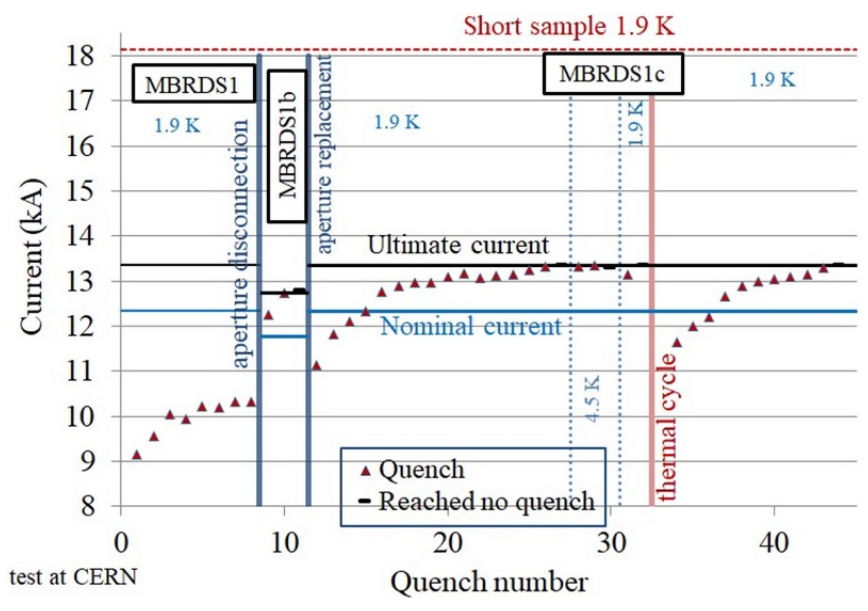

Figure 42. Training of the recombination dipole short model.

double aperture magnet. A second aperture was assembled with a new coil, and the magnet reached ultimate current in the nominal configuration (two aperture in series). After thermal cycle, ultimate current was reached again but with a non negligible retraining in one coil. The origin of this training will be investigated through magnet disassembly and visual inspection of the coils.

Concering field quality, room temperature measurements at $\pm 10 \mathrm{~A}$ allowed to prove the critical compensation of the coil asymmetry, magnetic cross-talk and iron geometry, see table 4: the optimization of field quality was proven within ten units for $b_{3}$ and $b_{5}$. The missing part towards the target of \pm 3 units (for $b_{3}$ ) and \pm 1 unit (for $b_{5}$ ) was found to be due to a missing shim of $0.125 \mathrm{~mm}$ in the midplane, removed to compensate for a excess in coil size.

The test of MBRDS1c had magnetic measurements at $1.9 \mathrm{~K}$, giving a saturation component of the $b_{3}$ multipoles of about 5 units, in line with the simulations. The strain measurements proved the absence of pole unloading at ultimate current. The protection system based on quench heaters was also validated in the second test.

\subsection{Design changes}

There were few iterations on the magnet design. The collars were initially separated from the nose (see figure 43); in the third aperture built to assemble MBRDS1c [54] it has been decided to include them in the collars to reduce the piling-up of tolerances, and to minimize the possibility of misplacement during assembly. The second iteration was done to optimize the area around the layer jump, a critical part of the design of this magnet (see next section). The third iteration was done on the iron shape: an ellitical shape was adopted to reduce the saturation component of $b_{3}$. This required an additional component for the cold mass assembly in the circular stainless steel shell (see the fillers in orange, figure 43). For the prototype and series, it has been decided to remove this component to reduce the cost and ease the assembly, and adopt the shape shown in figure 40.

The circular hole for the heat exchanger, present in a previous cross-section, was replaced in a very early phase of the project with an elliptical one. The main reason is that the cooling scheme was changed from heat exchanger to direct cooling, and therefore a $200 \mathrm{~cm}^{2}$ of free cross-section in the magnet was needed for heat extraction. Part of this surface was obtained through the elliptical shape of the hole.

\subsection{Setbacks and open issues}

The most critical issue for the magnet performance has been the design and the assembly of the layer jump that goes to the connection leads. The cable is kept in place via a G11 box as in the LHC main dipole, but there is no outer layer; therefore this box has to go through an opening in the collars that weakens the structure in the connection side, just before the coil heads. The first aperture had a short in this region after collaring; visual inspection revealed no trace of the short, and after an insulation reinforcement and a second collaring the short disappeared. The same aperture, and coil, was limiting performance at $10 \mathrm{kA}$, i.e. about half of short sample (see figure 42). After disassembly, the layer jump box was found to be broken and about half of the strands of the cable were cut during the collaring, see figure 44 , thus justifying the severe magnet performance limitation. The third aperture, build to replace the first one, also showed a short circuit that was located at the cable exit, at the coil protection sheet. An iteration on the design of this region will be implemented in the prototype.

The second issue that was found was an excess of prestress in the coil heads, fracturing or breaking the end spacers. In the third aperture the first end spacer had a breakage leaving unprotected $5 \mathrm{~mm}$ of cable. The region was repaired by filling with charged epoxy. For the second assembly of the short model third aperture, a preassembly with Fuji paper has been used to determine the level of prestress in the coil heads and avoid these issues.

\subsection{Timeline and schedule}

The main milestones of the recombination dipole development are the followings:

- April 2014: beginning of the design study;

- September 2016: tender for the short model awarded to ASG superconductors with contract start in November 2016;

- March 2018: beginning of coil winding for the short model;

- October 2018: tender for the prototype with option on the series attributed to ASG, with contract start in March 2019;

- February 2019: test of the short model, including disconnection of the faulty aperture;

- Summer 2019: fabrication of the third aperture of the short model;

- January 2020: tooling preparation for prototype.

The rate assumed for the schedule is a very conservative three magnets per year at full speed. This is done via one coil production line, taking 3 months for coil construction, 2 months for collaring and 2 for yoking. The cold mass is done 
Table 4. Magnetic measurements at room temperature of short model, straight part, with and without yoke. Multipoles (10 ${ }^{-4}$ relative to main component) given at $35 \mathrm{~mm}$ reference radius.

\begin{tabular}{|c|c|c|c|c|}
\hline \multirow[b]{2}{*}{ Multipole } & \multicolumn{2}{|c|}{ Without yoke } & \multicolumn{2}{|c|}{ With yoke } \\
\hline & Ap. 1 & Ap. 2 & Ap. 1 & Ap. 2 \\
\hline$b_{2}$ & 209 & -205 & 12.79 & -9.41 \\
\hline$b_{3}$ & 81.0 & 81.8 & 9.17 & 10.0 \\
\hline$b_{4}$ & -8.97 & 10.3 & 2.06 & -0.38 \\
\hline$b_{5}$ & -0.01 & 3.06 & 6.95 & 9.30 \\
\hline$b_{6}$ & -2.96 & 2.98 & -1.72 & 1.68 \\
\hline$b_{7}$ & -0.34 & 0.31 & -0.31 & 0.00 \\
\hline$a_{2}$ & 1.03 & 2.40 & 2.43 & 4.03 \\
\hline$a_{3}$ & -2.84 & -2.85 & -2.39 & -1.83 \\
\hline$a_{4}$ & 1.16 & -0.13 & 0.95 & -0.62 \\
\hline$a_{5}$ & 2.42 & 1.59 & 1.67 & 1.40 \\
\hline$a_{6}$ & 0.57 & 1.93 & 0.46 & 1.63 \\
\hline$a_{7}$ & 1.67 & 1.64 & 1.02 & 1.14 \\
\hline
\end{tabular}

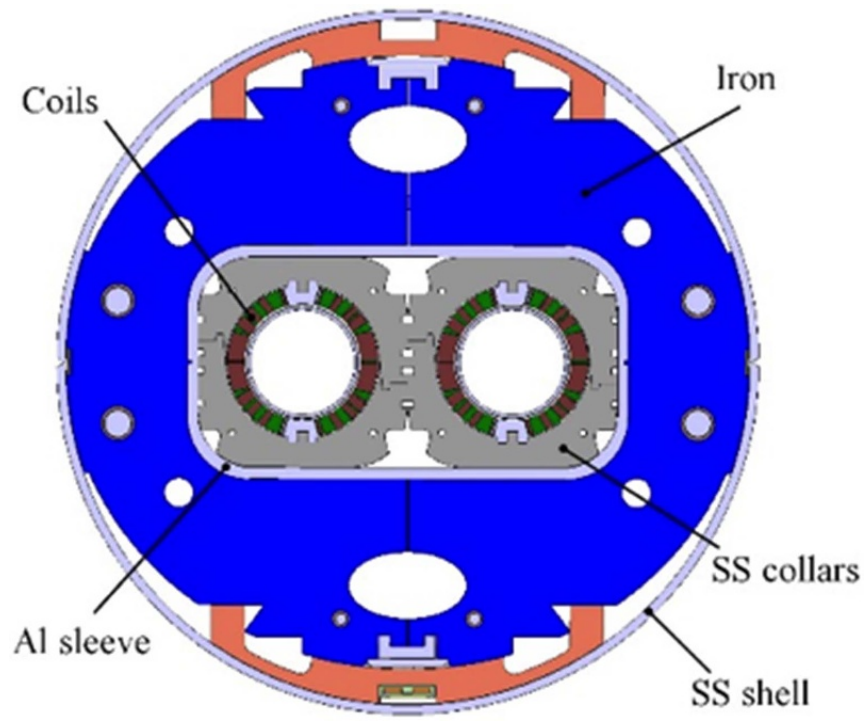

Figure 43. First cross-section of the recombination dipole D2.

at CERN, where the orbit correctors based on CCT technology (see section 7) are included. Due to the magnet length, it was not possible to find a station to test it vertically. This causes a very long feedback loop in case of issues during the test, namely 1 year from test to test in case of disassembly up to the level of the collared coil.

\section{The nested dipole correctors}

\subsection{Accelerator requirements}

The nested orbit correctors are single aperture dipoles with $150 \mathrm{~mm}$-diameter bore and a $2.5 \mathrm{~T} \mathrm{~m}$ nominal integrated field, both in horizontal and vertical direction (short version MCBXFB) and $4.5 \mathrm{~T} \mathrm{~m}$ nominal integrated field, both in horizontal and vertical direction (long version MCBXFA). The main function of these magnets is the correction of the misalignment of the triplet. The magnets have two different

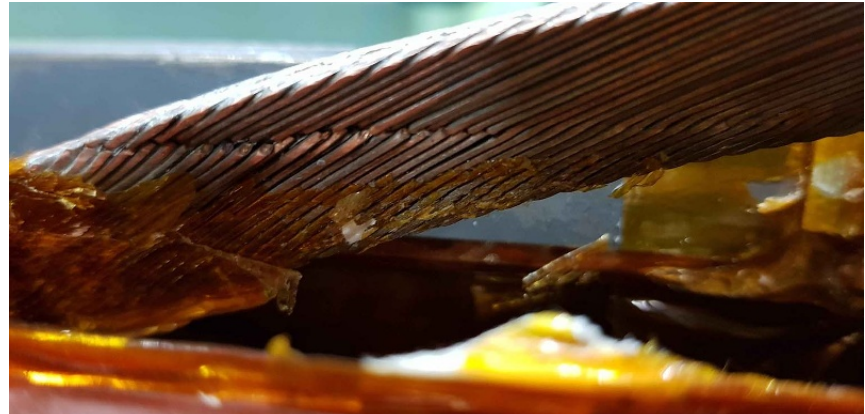

Figure 44. The damaged magnet lead after disassembly in MBDRS1.

lengths: the MCBXFB is $1.5 \mathrm{~m}$ long, and has to be assembled in the Q2a and Q2b cold masses (one per cold mass, see figure 1). The magnet MCBXFA is $2.5 \mathrm{~m}$ long, and has to be assembled in the corrector package cold mass. Besides correcting the orbit error due to the triplet misalignment, MCBXFA also contributes to open the crossing angle in the IP. Each dipole is individually powered, and the magnet shall operate at any combination vertical/horizontal dipole, with both directions of the field. Field quality requirements are set at $7 \mathrm{TeV}$ energy, with all multipoles at reference radius of $50 \mathrm{~mm}$ below 5 units with the exception of $b_{3}$, for which a larger tolerance of 20 units is accepted. No requirements are given on the saturation of the main dipolar component that can be compensated via the power converter. No requirements are given on the field quality at injection as for all the IR magnets. There are three magnets per IP side, each one having two circuits, for a total number of 24 power converters. To optimize the cost, $2 \mathrm{kA}$ is set as a maximum value for the nominal current. The most exposed parts of the magnet have to resist to $30 \mathrm{MGy}$ dose over the HL-LHC lifetime.

\subsection{Design features}

In order to satisfy the constraint on the current, a double layer coil based on a small Rutherford cable was used, namely a 


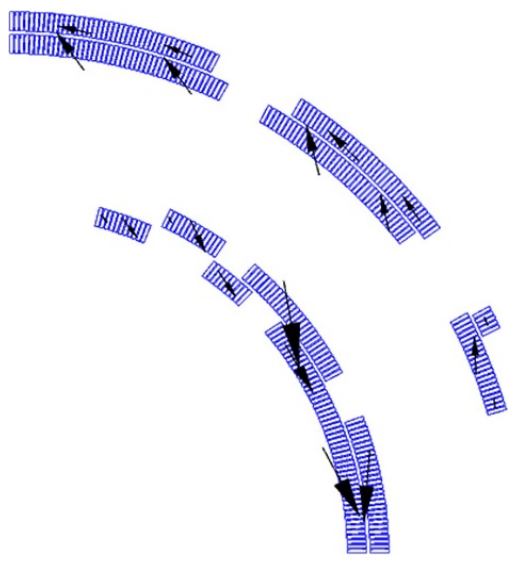

Figure 45. Cross-section of the nested dipole corrector coil, and electromagnetic forces with nominal current in both dipoles (one quarter shown).

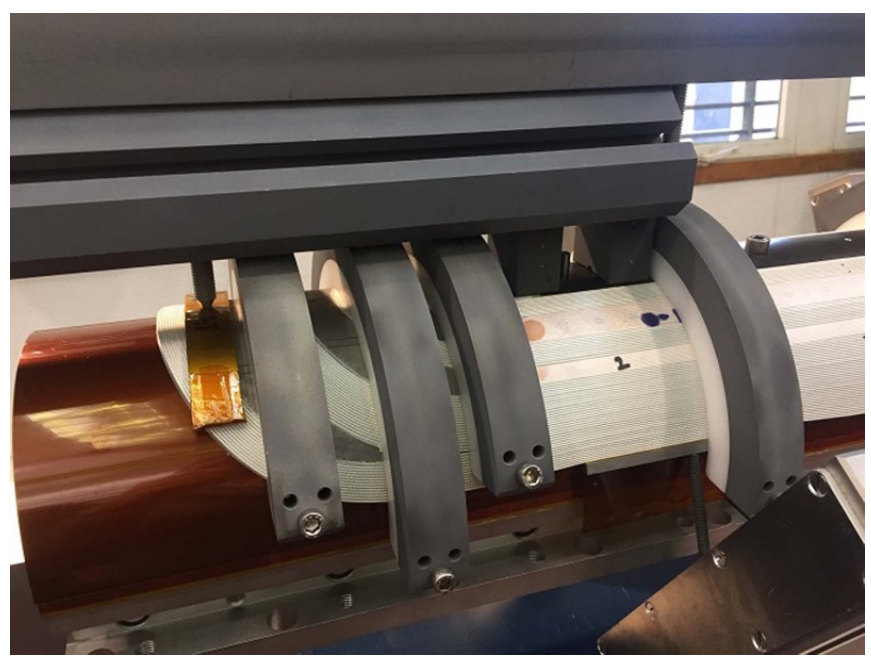

Figure 46. Coil head during winding.

$4.37 \mathrm{~mm}$-width cable, with 18 strands and a $0.48 \mathrm{~mm}$-diameter wire. The strand was already used for the cable of MQM and in the LHC [1]. The cable was developed in the framework of the S-LHC project for an upgrade of the orbit corrector of the triplet.

With such a large aperture and such a small width cable, $\sim 70$ to $\sim 100$ turns are needed for each layer (see figures 45 and 46, and table 1) and therefore the option of an impregnated coil was taken. The same technology of $\mathrm{Nb}_{3} \mathrm{Sn}$ was adopted, namely a braided fiberglass insulation and CTD-101 K resin [58-60].

The vertical dipole coil has three blocks (inner layer) and three blocks (outer layer), for a total of 140 turns. The horizontal dipole coil has also three blocks (inner layer) and three blocks (outer layer), for a total of 191 turns (see figure 45). A large contribution to the field comes from the iron, namely $34 \%$ for the inner layer and $64 \%$ for the outer layer. Therefore the current density in the outer dipole is about $15 \%$ smaller than in the inner dipole. Current densities are of the order of $300 \mathrm{~A} \mathrm{~mm}^{-2}$, i.e. $30 \%$ lower than in the main HL-LHC IR magnets (see figure 5).

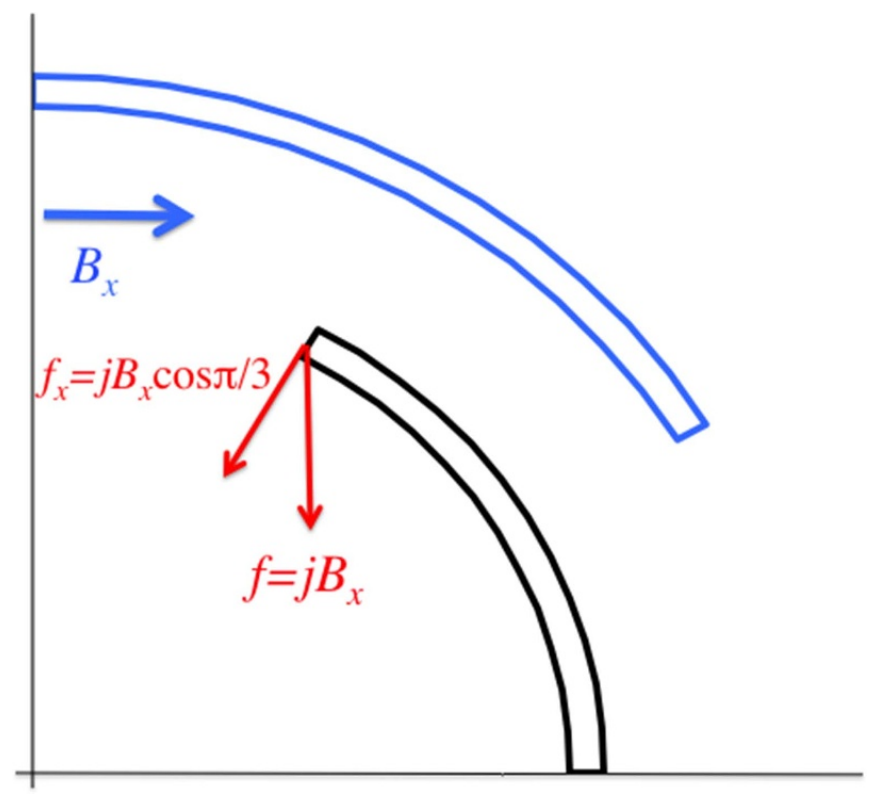

Figure 47. Electromagnetic forces induced by outer dipole acting on the inner dipole coil (neglecting inner dipole field effect).

Each coil provides a bore field of 2.15/2.26 T (vertical/horizontal). In single dipole configuration, the peak field is 2.54/2.65 T (vertical/horizontal); in combined mode, a bore field of $3.12 \mathrm{~T}$ field with an inclination of $46.4^{\circ}$ is provided, with a peak field of $4.3 \mathrm{~T}$ in the inner layer of the vertical coil, corresponding to a loadline fraction of 0.51 . In single powering mode, there is a $25 \mathrm{MPa} / 40 \mathrm{MPa}$ accumulated stress in the midplane of the inner/outer dipole. When both dipoles are powered the precompression required to avoid any coil movement increases from 25 to $45 \mathrm{MPa}$ for the inner dipole. Therefore the required precompression to avoid pole unload at nominal current is not critical.

The first non trivial element of the nested corrector design is that when both dipoles are powered, a force directed towards the bore is applied to the coil pole of the inner dipole, see figure 47 illustrating the simplified case that neglects the inner field contribution. Using the map of electromagnetic forces computed on the actual cross-section (see figure 45), one finds a maximum shear stress between the coil and the pole/wedge of $3.5 \mathrm{MPa}$. This shear stress shall be compensated by a coil precompression at $1.9 \mathrm{~K}$ of $60 \mathrm{MPa}$, providing a residual compressive azimuthal stress of $15 \mathrm{MPa}$ in any operational conditions with nominal currents. This compressive stress prevents coil displacements towards the bore when both dipoles are powered.

Note that in the initial design, a radial gap of $3 \mathrm{~mm}$ was left between the inner bore and the coil to allow sliding a tube to prevent inward movements of the coil. Therefore, the inner coil radius is $78 \mathrm{~mm}$ and not $75 \mathrm{~mm}$. The option of the inner support tube was abandoned in an early phase of the project, considering that the coil preload was sufficient to avoid inward movements of the coil.

A second non trivial element of the design is that when both dipoles are powered at nominal curernt there is a $140 \mathrm{kN} \mathrm{m} / \mathrm{m}$ 


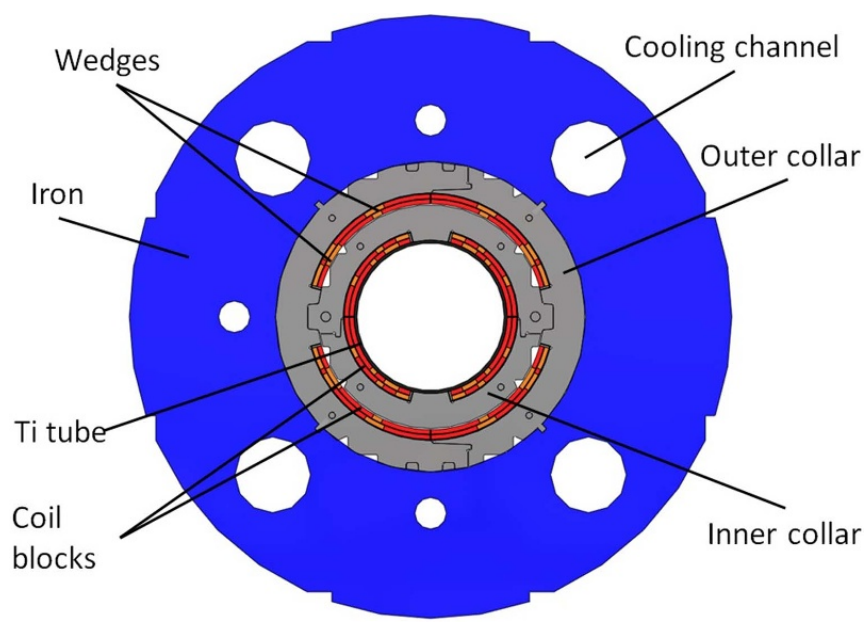

Figure 48. Cross-section of the nested dipole corrector coil.

torque. To manage this large torque a double collared structure with a mechanical lock between the horizontal and the vertical apertures has been developed (see figure 48). Collars have a 25/31 $\mathrm{mm}$ thickness for the inner and outer dipoles respectively, and the mechanical lock is present on the straight part of the magnet; there is a $1 \mathrm{~mm}$ nominal gap between the horizontal dipole coil and the inner dipole collars. The coil heads have no mechanical lock, and they rely on the lock in the straight part, working in cantilever, and axial load given by the endplate on the coil heads. In these conditions, in absence of friction, the maximum movement in the inner coil due due this unsupported torque is order of $0.2 \mathrm{~mm}$. Hence, coils heads are also precompressed by round collars to prevent the coil motion in the ends.

The second issue that was found was an excess of prestress in the coil heads, fracturing or breaking the end spacers. In the third aperture the first end spacer had a breakage leaving unprotected $5 \mathrm{~mm}$ of cable. The region was repaired by filling with charged epoxy. For the second assembly of the short model third aperture, a preassembly with Fuji paper has been used to determine the level of prestress in the coil heads and avoid these issues.

Due to the large number of turns, the inductance is large enough (between 50 and $230 \mathrm{mH}$ for long/short and inner/outer dipole, see table 1) to require an active protection system. The protection is guaranteed by energy extraction on a $0.15 \Omega$ resistor, with an hotspot temperature below $250 \mathrm{~K}$. Quench heaters were initially considered, but discarded in an early phase of the project to reduce complexities in the coil manufacturing and in the magnet assembly. The iron saturation is asymmetric in the configuration with simultaneous powering, (see figure 49).

\subsection{Design validation via power tests}

The first prototype (1.5 m long corrector MCBXFBP1) was initially tested only with the inner dipole assembly; the magnet reached ultimate current without training (see figure 50). The outer dipole dipole was then added to the magnet asssembly, and in the final configuration the outer dipole also reached ultimate current with 11 quenches. In combined powering mode,

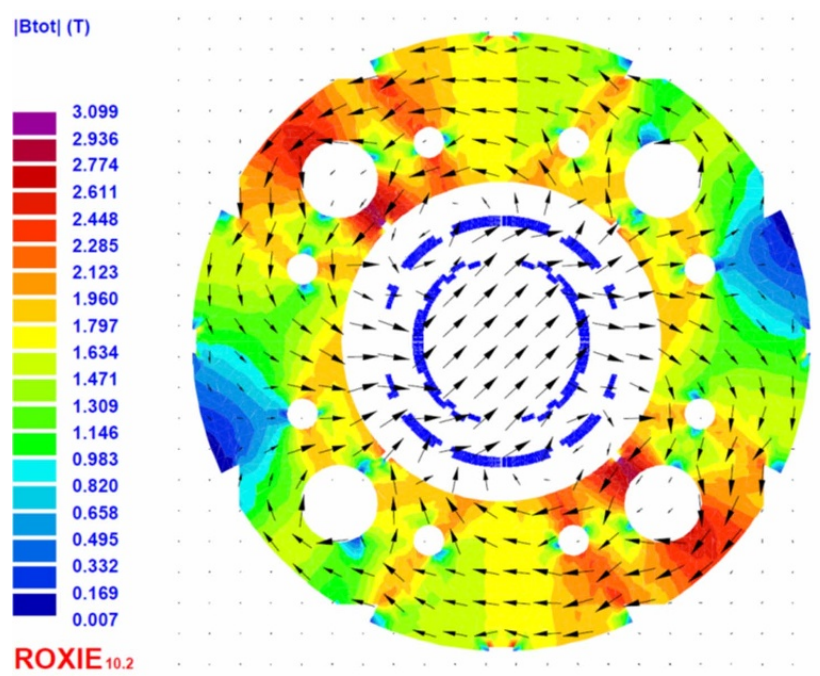

Figure 49. Field map in the iron during simultaneous powering of both dipoles at nominal field.

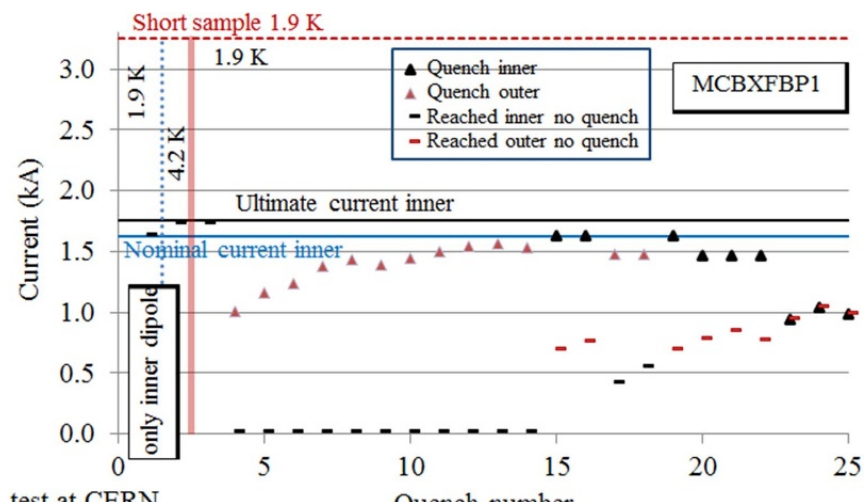

Figure 50. Training of MCBXFBP1.

only $50 \%$ of the product between the horizontal and vertical field was reached, with most of the quenches in the inner dipole (two only in the outer) and localized in the coil heads.

After an optimization of the shimming, aiming at azimuthal compression also in the coil heads, the magnet reached reached nominal current in both planes after 10-20 quenches (see figure 51). This phenomenology confirmed that the performance issue for the first assembly was not relative to the map of electromagnetic forces in the inner dipole, but rather to the torque in the coil ends.

After this training, the magnet could operate in the opposite quadrant (negative current in both dipoles) at nominal current. To operate in the other two quadrants, a further training of $\sim 10$ quenches was required. This training for reaching positive horizontal dipole and negative vertical (or vice versa) prevents to operate with the dipoles having the same sign. The inprepretation is that training is setting the inner dipole coil heads in a position more favorable to the torque. Therefore the training for one sign of the torque 'detrains' the training in the other sign.

Possible limitations of these features to operation are being studied; at the same time, imporovement in the design are 


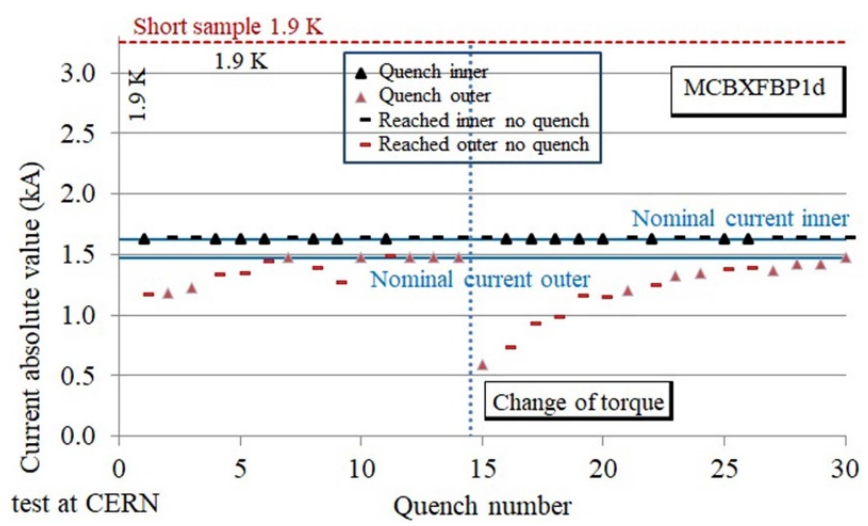

Figure 51. Training of MCBXFBP1d.

under consideration, plus a check of the reproducibility of this behaviour.

\subsection{Design changes}

The first version of the magnet had a single layer coil; this was providing a simpler design, at the price of a higher loadline fraction $(\sim 0.65)$ and operational current $(\sim 3 \mathrm{kA})$. The $2 \mathrm{kA}$ limitation on power converter forced to go for a double layer design, allowing to decrease the loadline fraction at around $50 \%$ and getting more operational margin. This change was done in an early phase of the project (2015), i.e. well before the engineering phase.

The second design change concerned the position of the heat exchangers. As for the D1 (see section 4) initially the holes for the long corrector MCBXFA were at $90^{\circ}$ and at $270^{\circ}$, plus two additional at $0^{\circ}$ and at $180^{\circ}$ not to break the symmetry of the corrector. In 2016 it was realized that the interconnection geometry imposed the alignment of heat exchanger among the triplet, the corrector package and the D1. Therefore all the heat exchanger holes were positioned at $45^{\circ}, 135^{\circ}, 225^{\circ}$ and $315^{\circ}$ as shown in figure 40 .

The third change concerned the protection system. Initially simulations showed that the short magnet MCBXFB could be protected by quench propagation, without the need of energy extraction. Results of the first tests showed that quench propagation was on the edge of ensuring a hotspot temperature below $300 \mathrm{~K}$, and therefore an extraction system has been included.

\subsection{Setbacks and open issues}

The most critical part is the support of the coil heads. The mechanical lock is present only in the straight part, and an adequate level of precompression in the coil head has to be used to avoid training.

The precompression in the straight part was shown to be effective to prevent the motion of the inner coil towards the magnet centre under the electromagntic forces. Therefore the option of an inner tube to support the coil from inside the aperture has been discarded.

\subsection{Timeline and schedule}

The main milestones of the nested dipole corrector development are the followings:

- July 2014: first conceptual design based on double collaring and single layer;

- June 2015: double layer design;

- January 2016: beginning of engineering design;

- February 2017: mechanical model;

- September 2017: beginning of coil winding;

- Fall 2018: collaring of the prototype;

- January 2019-August 2019: test of the first prototype with succesive iterations on the magnet assembly parameters.

\section{The canted $\cos \theta$ dipole correctors}

\subsection{Accelerator requirements}

The D2 orbit correctors are double aperture dipoles with $105 \mathrm{~mm}$-diameter bore and a $5 \mathrm{~T} \mathrm{~m}$ nominal integrated field. The magnet function is to open the crossing angle in the IP, to close any possible orbit bump in the crab cavities located between D2 and Q4, and to correct the orbit error due to misalignent of the triplet. Each aperture is individually powered. Field quality requirements are set at $7 \mathrm{TeV}$ energy, with all multipoles at reference radius of $35 \mathrm{~mm}$ within \pm 3 units with the expection of $b_{3}$, for which a larger tolerance of \pm 10 units is accepted. No requirements are given on the saturation of the main dipolar component that can be compensated via the power converter. No requirements are given on the field quality at injection as for all the IR magnets. Two set of magnets are needed for each D2: an horizontal and a vertical dipole. The most exposed part of the magnet have to resist to 6 MGy dose over the HL-LHC lifetime.

\subsection{Design features}

Since the two apertures can be powered in any configuration, the magnetic cross-talk cannot be compensated by asymmetric coil design as in D2. Therfore, the field quality constraints set a limit on the maximum field, which should be not too far from the iron saturation levels. For D2 correctors, a bore field of 2.6 T has been selected, for a $1.9 \mathrm{~m}$ magnetic length (see table 1). As in the LHC [1], horizontal and vertical dipoles are alternatively coupled in the same magnet to reduce the crosstalk between apertures. All magnets have the same configuration, i.e. horizontal dipole is always on the right side of the magnet seen from connection side. Inside the cold mass, the two correctors are installed with the connection in opposite sides, to have a horizontal and a vertical dipole on each beam tube.

The initial layout was based on a standard sector coil; then it was decided to adopt a canted $\cos \theta \operatorname{coil}[61,62]$. According to this idea [64], two tilted solenoids are wound in a metallic former, with opposite inclination (see figure 52, where the two short windings are shown before assembly). When the two coils are assembled around the same aperutre, the solenoidal 


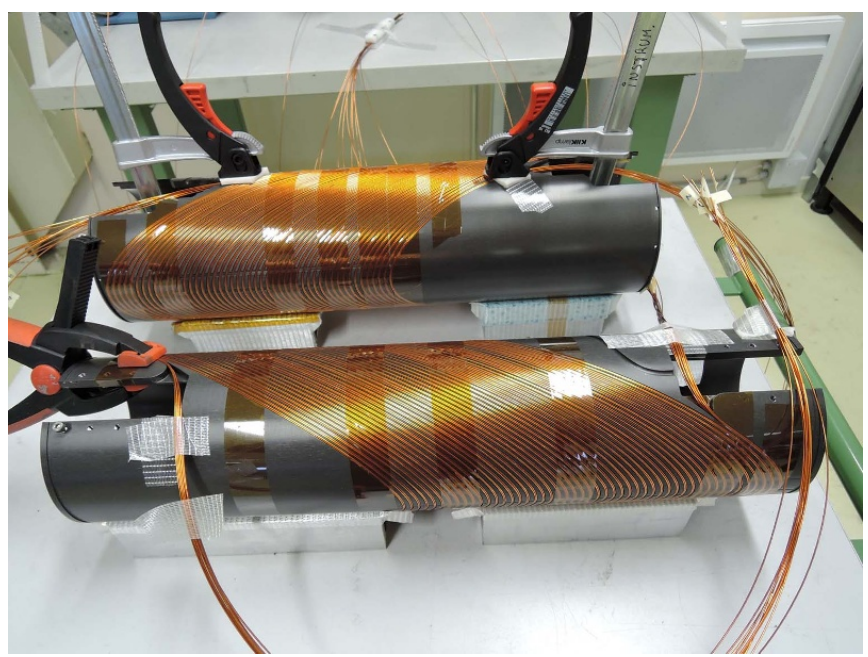

Figure 52. Two two tilted solenoids of the short model of the canted corrector before being inserted one into the other.

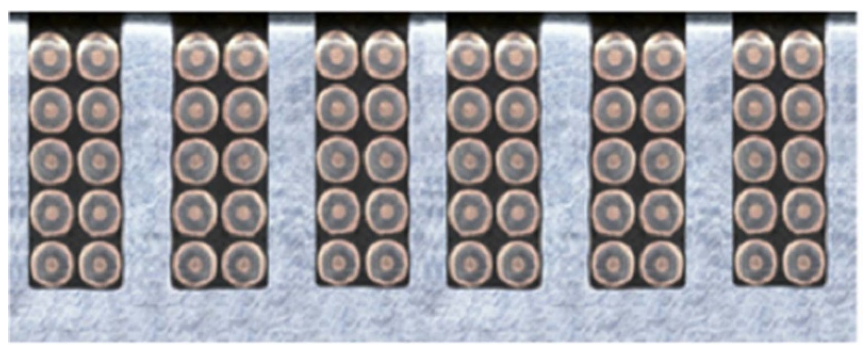

Figure 53. Position of the wire in the grooves of the formers.

field is canceled and a pure dipolar field is left. The design has the advantage of requiring a simplified winding machine, and very little tooling and components for the assembly: no collars, no press, no wedges, no end spacers. On the other hand, a large fraction of the conductor is used to generate a solenoidal field that is canceled by the other winding, and there is no way of prestressing the conductor in the groove. Moreover, no wedges means no possibility of fine tuning the field quality. This design was considered to be ideal for a low to intermediate field application (i.e. 2-4 T) as the D2 corrector, since the conductor is not a relevant part of the cost.

The loadline fraction was set to be lower than $50 \%$ as a general guideline for the correctors. This was realized, see $[61,62]$, with a ten turns per slot winding (see figure 53) of a $0.825 \mathrm{~mm}$-diameter $\mathrm{Nb}-\mathrm{Ti}$ wire (same wire of the LHC outer dipole cable). Each wire is insulated by wrapping a polyimide tape, for a total diameter of the insulated wire of $1 \mathrm{~mm}$. The slots in the former are $2.1 \mathrm{~mm}$ wide times $5.2 \mathrm{~mm}$ deep to allow a relatively easy but tight winding. The slots make 365 turns over the $2 \mathrm{~m}$ long magnet, with a $30^{\circ}$ angle with respect to the beam axis. The former material is $\mathrm{Al}$, hard anodized to reinforce insulation. The two formers with opposite tilted solenoids are impregnated together with CTD-101 K to ensure a mechanical stability of the coil.

The magnet has the advantage of a very low operational current (400 A), but the drawback of a large inductance $(\sim 1 \mathrm{H}$ per

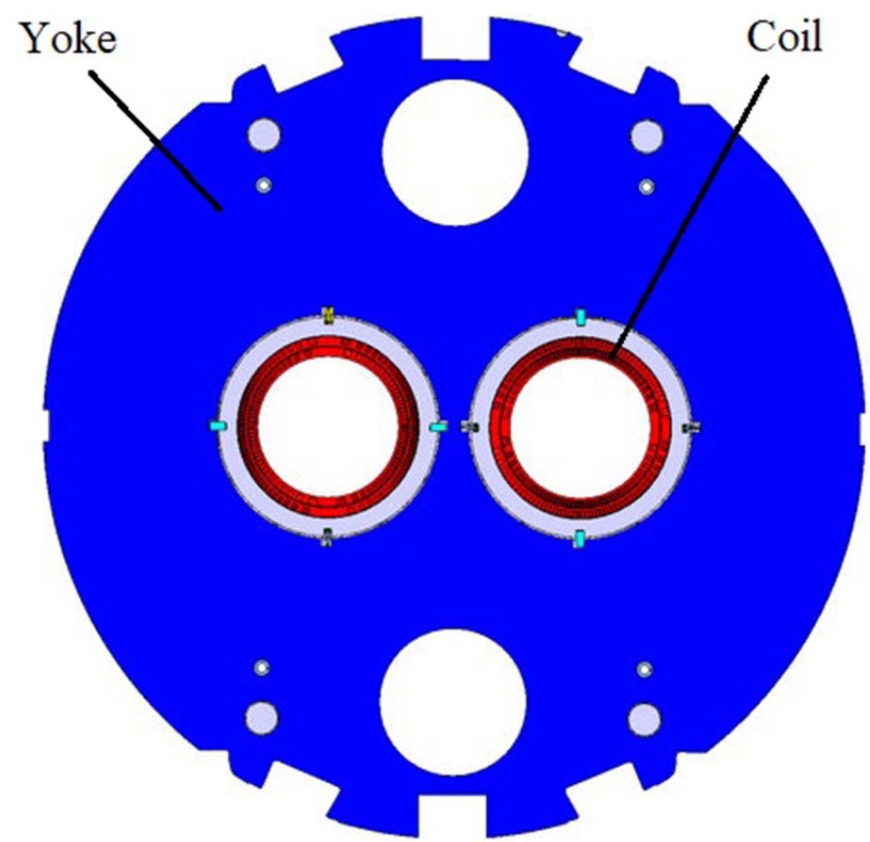

Figure 54. Cross-section of the MCBRD magnet.

aperture). A maximum hotspot temperature of $200 \mathrm{~K}$ is set for these correctors. The protection is ensured via a dump resistor and the associated quench back thanks to the eddy currents in the $\mathrm{Al}$ former induced by $\mathrm{d} I / \mathrm{d} t$. Indeed, this magnet design is a different paradigm with respect to $\cos \theta$ and block coil magnets and in principle can tolerate a larger current density and a larger energy density on the coil, since the former can contribute to the protection through eddy currents through its enthalpy. A schematic cross-section of the two apertures with the iron yoke is given in figure 54 .

The magnet design was developed at CERN, with a short model and a $2 \mathrm{~m}$ long prototype program. In 2018, China agreed with CERN to have the 12 series magnets as an inkind contribution, plus a protoype, based on CERN design. A protoype was completed in Western Superconducting Technologies (WST), Xi' an and tested in Institute of Modern Physics (IMP), Lanzhou.

\subsection{Design validation via power tests}

Even though the D2 corrector is only $2 \mathrm{~m}$ long, since CERN had no previous experience in this design, it was decided to manufacturing a double aperture $0.5 \mathrm{~m}$ long model [61]. Both apertures reached ultimate current with one quench, showing perfect memory after thermal cycle. The second aperture was powered up to $75 \%$ of short sample limit (see figure 55).

Then, a full size prototype with $2 \mathrm{~m}$ long coils was manufactured. The protoype second aperture had similar performance of the short model, but the first aperture required a long training [63], with 20 quenches to nominal current and another ten quenches to ultimate current (see figure 56). A third aperture was manufactured to replace the first aperture. In this test, the third aperture required three quenches to reach ultimate current, and the previously tested aperture reached ultimate 


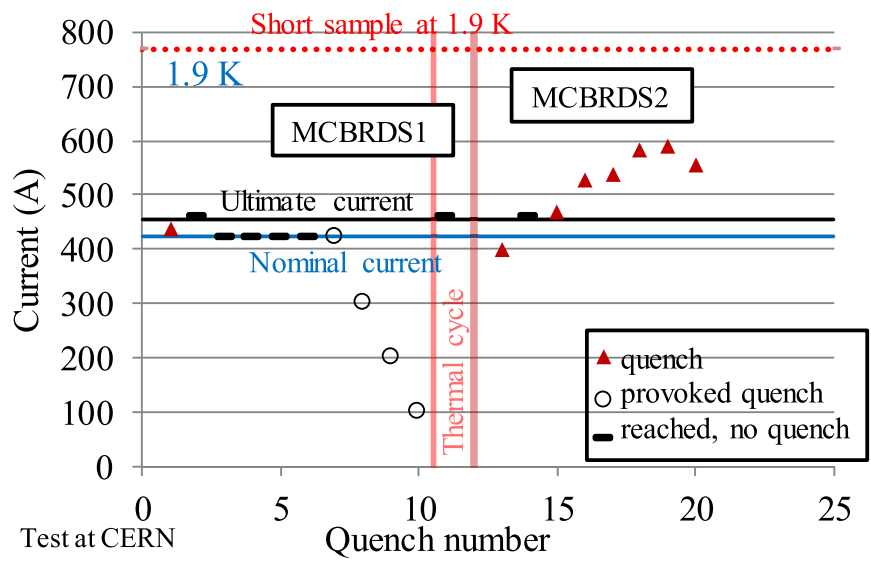

Figure 55. Training performance of D2 corrector short model.

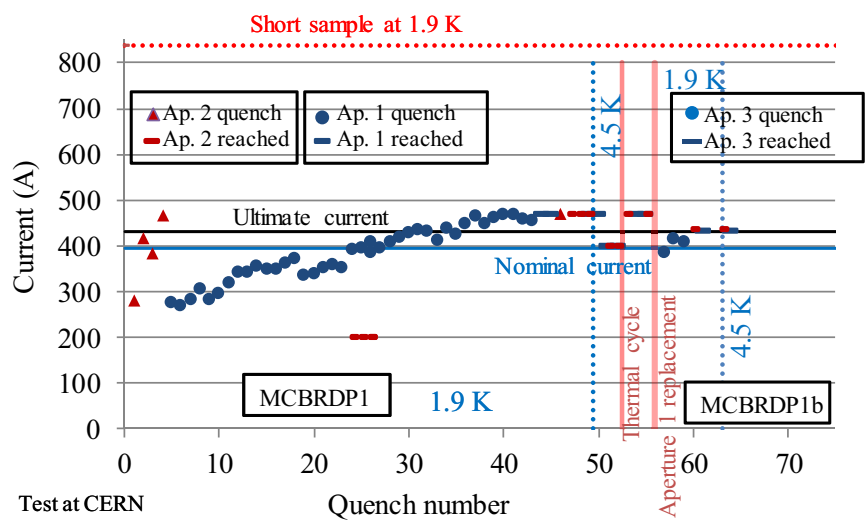

Figure 56. Training performance of $\mathrm{D} 2$ corrector first prototype.

wihtout retraining. Both apertures reached ultimate current also at $4.5 \mathrm{~K}$.

The prototype built in China (see figure 57) reached the ultimate current with a long training, similarly to the first aperture manufatured at CERN. The impregnation is considered to be one of the possible aspects related to this long training. A few quenches were needed after thermal cycle to reach ultimate current at $4.1 \mathrm{~K}$. The maagnet was then tested at CERN at $1.9 \mathrm{~K}$, requireing no further training to reach ultimate current. At the moment of writing the series of 12 magnets is under construction in BAMA, Shozou.

Quench protection proved that quench back is the dominating mechanism. It can be initiated by a dump resistor of $1.4 \Omega$; quench back increases the speed of discharge (i.e. the increase of resistance of the coil) by a factor of three (see figure 58).

The field quality was measured before assembly in the yoke and all harmonics were shown to be well within 3 units. After assembly a 10 units $a_{3} / b_{3}$ component was found due to the keys needed for coil alignment in the iron (see figure 54). A design correction is being implemented in the next magnets.

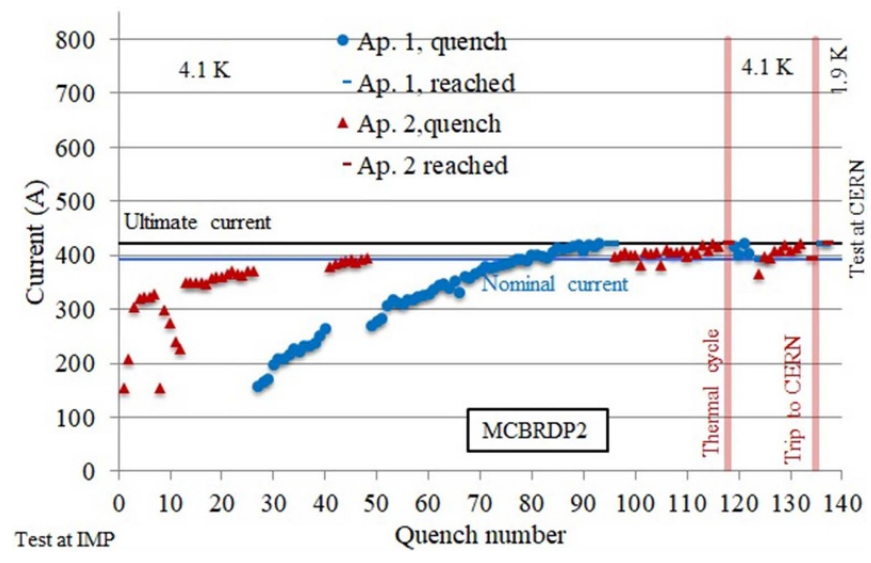

Figure 57. Training performance of $\mathrm{D} 2$ corrector first prototype.

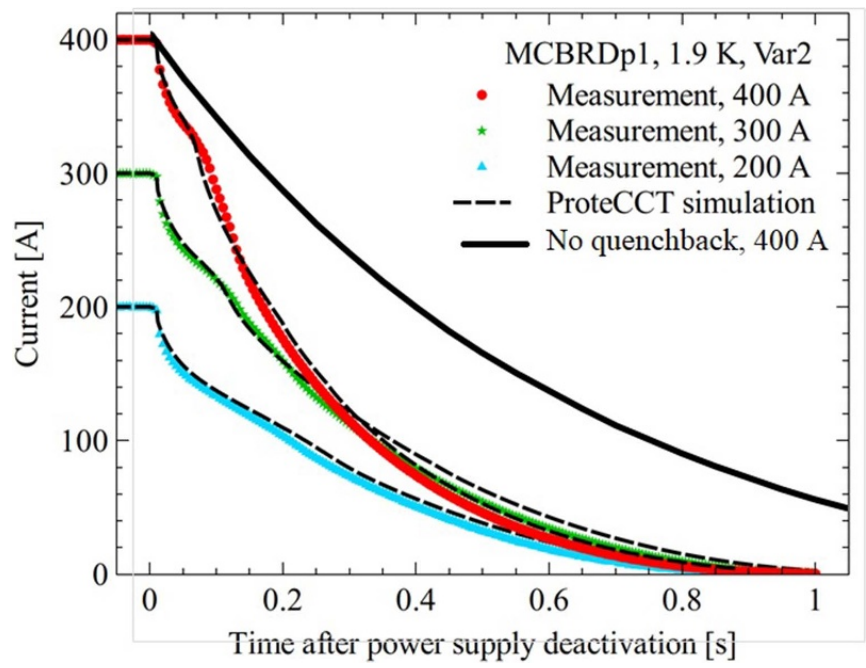

Figure 58. Discharge during quench of the MCBRD corrector, measurements versus simulation, and case without considering quenchback.

\subsection{Design changes}

The only design change was the development a special tool to wind the ten cables in the groove at the same time (see figure 59). This considerably simplified the coil winding, that can be performed as fast as 1 day per layer.

\subsection{Setbacks and open issues}

Even though this technology was a prima for CERN the development had no setbacks. The only critical point is the slow training in virgin conditions, which is possibly related to the quality of the impregnation.

\subsection{Timeline}

The main milestones of the canted dipole corrector development are the followings:

- August 2014: beginning of the design study;

- August 2015: selection of the canted $\cos \theta$ design; 


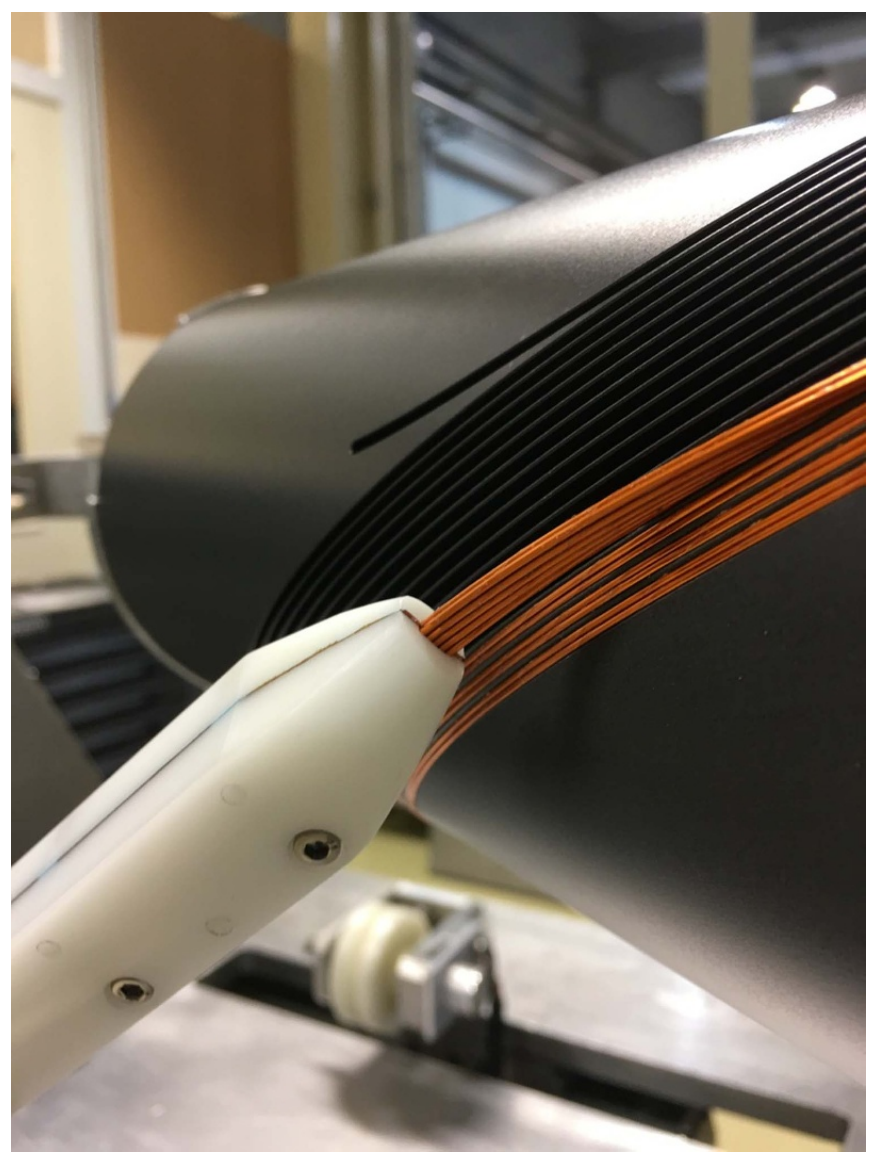

Figure 59. Tooling allowing to wind ten strands in one go.

- March 2017: beginning of collaboration and technology transfer to IHEP (Beijing);

- August 2017: test of the first aperture of the short model at CERN;

- May 2018: test of the second aperture of the short model at CERN;

- November 2018: test of the prototype at CERN;

- May 2019: beginning of prototype construction in WST (Xi'an);

- January 2020: test of the CERN prototype with one aperture replaced;

- March 2020: completion of the prototype in WST and test in IMP.

For this magnet the schedule constraint is given by the former manufacturing, that can take 2 weeks. The coil winding is quite fast, and can be done in 1 to 2 days. A production line with one winding machine, one impregnation system, and one asembly nech for the magnets can provide one magnet every 2 months

\section{The superferric high order correctors}

\subsection{Accelerator requirements}

The high order correctors are five types of magnets (quadrupole to dodecapole) needed to correct the tilt error of the triplet (skew quadrupole), and the high order field imperfections of the triplet and of the separation dipole (sextupole to dodecapole). The requirements in terms of integrated field (main field at $50 \mathrm{~mm}$ reference radius, integrated over the magnet length) are given in table 5 . They correspond to a maximum correction of an average tilt of the triplet of $\pm 5 \mathrm{mrad}$, and of the following nonilnearities in the triplet: \pm 6.8 units of sextupole, \pm 5 units of octupole, \pm 2.6 units of decapole, \pm 6 units of normal dodecapole and \pm 1.5 units of skew dodecapole.

The skew quadrupole is used from injection to top energy; the higher order corrector magnets are used only at top energy, after the beam squeeze. All these correctors are individually powered. Field quality requirements impose multipoles at reference radius below 100 units (i.e. below $1 \%$ of the main component). They have to be able to operate up to a $15 \mathrm{MGy}$ radiation dose.

\subsection{Design features}

Since the very beginning of the project, a superferric technology based on coils wound with $\mathrm{Nb}-\mathrm{Ti}$ wire around iron poles has been chosen (see figures 60 and 61). The design, carried out by INFN-Milano in LASA laboratory [67] relied on the development and construction of a similar superferric sextupole developed in 2011-2012 for the FAIR and for the S-LHC study by CIEMAT $[74,75]$.

The main magnet parameters are listed in table 5. As already discussed in section 2.1 , the magnets operate at a low loadline fraction of $0.25-0.45$. The peak field on the coil is between 1.5 and $3.6 \mathrm{~T}$; the coils are $150 \mathrm{~mm}$ long, with the exception of skew quadrupole and normal dodecapole whose length is $\sim 500 \mathrm{~mm}$. For all magnets except the quadrupole, an operational current not larger than $105 \mathrm{~A}$ is required to allowe reusing the LHC power converters, giving a significant cost reduction (total of 32 independent circuits). For the skew quadrupole a limit of $210 \mathrm{~A}$ is considered. To match this requirement, a Nb-Ti strand diameter of $0.5 \mathrm{~mm}$ has been selected ( $0.7 \mathrm{~mm}$ for the skew quadrupole) and the coils are made with 200-750 turns. The insulation is made with S2-glass braid, and coils are impregnated with CTD-101 K $[67,70]$.

Coils are kept in position via metallic wedges that are pushed radially on the iron poles. These wedges also provide the necessary mechanical support to balance the electromagnetic forces.

\subsection{Design validation via power tests}

The protection is done via energy extraction for the skew quadrupole, that has an inductance well larger than $1 \mathrm{H}$. For the other magnets quench propagation is enough to build up the required resistance to rapidly dump the current. A maximum hotpost temperature of $200 \mathrm{~K}$ is specified.

Sextupole, octupole and decapole full-size prototypes were built in LASA laboratory; dodecapole and skew quadrupole prototypes were built in SAES-RIAL (Italy) since their size was not compatible with LASA infrastructure. For all magnets, desgin and follow up were provided by LASA. 
Table 5. Main requirements and parameters of the high order correctors.

\begin{tabular}{|c|c|c|c|c|}
\hline Name & Unit & MQXSF & MCSXF MCSSXF & MCOXF MCOSXF \\
\hline Order $n$ & $(\operatorname{adim})$ & 2 & 3 & 4 \\
\hline Integrated strength & $(\mathrm{T} \mathrm{m})$ & 0.700 & 0.095 & 0.069 \\
\hline Coil lenght & $(\mathrm{mm})$ & 457 & 192 & 172 \\
\hline Gradient & $\left(\mathrm{T} / \mathrm{m}^{\mathrm{n}-1}\right)$ & 34.8 & 224 & 3680 \\
\hline Coil peak field & $(\mathrm{T})$ & 3.6 & 2.23 & 2.09 \\
\hline Strand diameter & $(\mathrm{mm})$ & 0.7 & 0.5 & 0.5 \\
\hline $\mathrm{N}$ turn/pole & $(\operatorname{adim})$ & 754 & 288 & 372 \\
\hline Current & (A) & 174 & 99 & 102 \\
\hline $\mathrm{J}$ overall & $\left(\mathrm{A} \mathrm{mm}^{-2}\right)$ & 314 & 308 & 317 \\
\hline Loadline fraction & (adim) & 0.44 & 0.31 & 0.31 \\
\hline Diff. inductance & $(\mathrm{mH})$ & 1530 & 213 & 220 \\
\hline Stored energy & $(\mathrm{kJ})$ & 30.8 & 1.72 & 1.55 \\
\hline Name & Unit & MCDXF MCDSXF & MCTXF & MCTSXF \\
\hline Order $n$ & (adim) & 5 & 6 & 6 \\
\hline Integrated strength & $(\mathrm{T} \mathrm{m})$ & 0.037 & 0.086 & 0.017 \\
\hline Coil lenght & $(\mathrm{mm})$ & 172 & 498 & 123 \\
\hline Gradient & $\left(\mathrm{T} / \mathrm{m}^{\mathrm{n}-1}\right)$ & 40480 & 585600 & 550400 \\
\hline Coil peak field & $(\mathrm{T})$ & 1.63 & 1.57 & 1.50 \\
\hline Strand diameter & $(\mathrm{mm})$ & 0.5 & 0.5 & 0.5 \\
\hline$N$ turn/pole & (adim) & 228 & 432 & 432 \\
\hline Current & (A) & 92 & 85 & 84 \\
\hline $\mathrm{J}$ overall & $\left(\mathrm{A} \mathrm{mm} \mathrm{m}^{-2}\right)$ & 286 & 264 & 261 \\
\hline Loadline fraction & (adim) & 0.26 & 0.27 & 0.27 \\
\hline Diff. inductance & $(\mathrm{mH})$ & 120 & 805 & 177 \\
\hline Stored energy & $(\mathrm{kJ})$ & 0.668 & 3.63 & 0.732 \\
\hline
\end{tabular}

Power tests at $4.2 \mathrm{~K}$ were systematically carried out at LASA laboratories on all prototypes. Verification at $1.9 \mathrm{~K}$, together with field quality measurements were done at CERN. Results are shown in figures 62-66. The sextupole prototype reached operational current without training (see figure 62) and was powered up to $65 \%$ of short sample [68]. The octupole required few quenches to reach operational current (see figure 63) and was powered up to $63 \%$ of short sample [69]. Decapole and dodecapole reached operational current with one quench (see figures 64 and 65) [71, 72]. In all cases no retraining was observed after thermal cycle.

The skew quadrupole had a non negligible training, present also after thermal cycle (see figure 66). An iteration on the support of the coils was done, and the second assembly showed a more limited training and perfect memory. Note that in the quadrupole not only the forces are larger, but also that the loadline fraction is $44 \%$ compared to $26 \%-31 \%$ as in the higher order magnets.

The field quality was measured in sextupole, octupole and decapole, with results below 10 units, i.e. ten times smaller than the acceptance tolerances. The saturation was measured to be in agreement with the $3 \mathrm{D}$ magnetic model [73].

\subsection{Design changes}

A first change was introduced at the beginning of the project, namely to fit the $120 \mathrm{~A}$ limit for the ultimate current in order to reuse the LHC power converters. The first prototype sextupole did not include this constraint, and had a slightly larger current [67]. In the iteration of the design, additional turns were added to fit the current constraint.

The second change concerned an iteration on the material for the coil box; three different materials were tested, namely 3D printed ULTEM, Duratron, and BT resin S2 reinforced. After several tests, the latter was selected [64].

A third change was the $50 \%$ increase of the field integral requirements for sextupole, octupole and decapole to cope with larger unallowed low order multipoles in the triplet (see section 3 ). The field integral increase was obtained via a $\sim 30 \%$ longer magnetic length. The space was recovered by reducing the length of the skew quadrupole, that revealed to be overdimensioned with respect to alignement tolerances and whose requirement was reduced by $30 \%$ from $1 \mathrm{~T} \mathrm{~m}$ to $0.7 \mathrm{~T} \mathrm{~m}$ integrated gradient (see table 5).

\subsection{Setbacks and open issues}

The first power test of the quadrupole corrector was interrupted after the 15th quench (see figure 66) due to the appearance of an electrical interturn short provoking the loss of electrical integrity (see figure 67). The origin of the problem was traced back to a weakness in the insulation of the coil at the location of the wire exit. After this finding, the design of the insulation at the coil lead and the design of all connection plates were modified in all magnets, even though the previous produced coils did not show weakness. The test of the second assembly was succesful (see figure 66). 


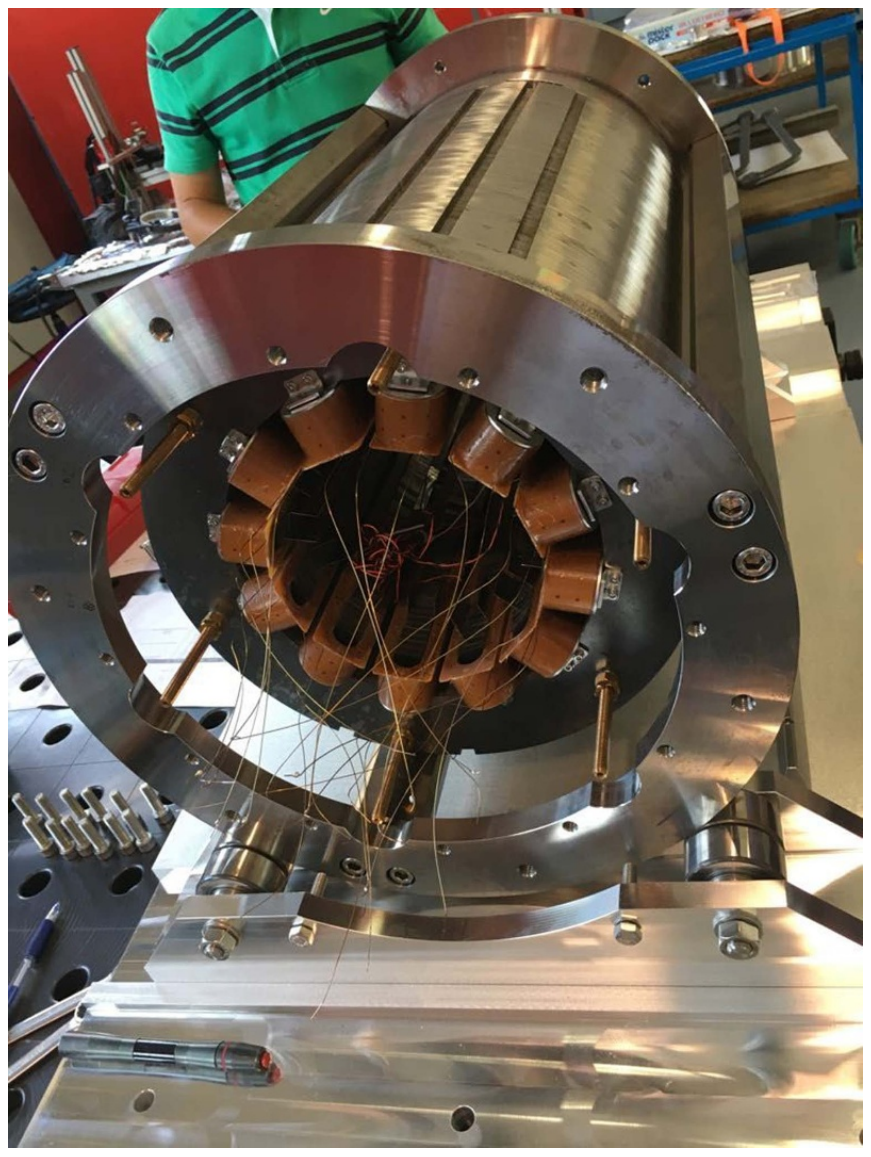

Figure 60. Assembly of the dodecapole prototype coils in the iron laminations.

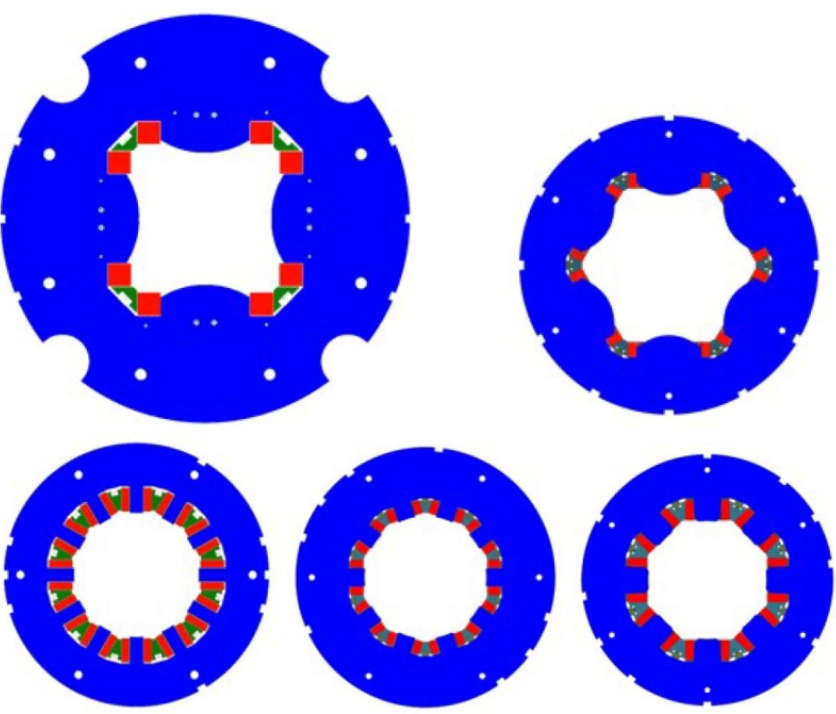

Figure 61. Cross-sections of quadrupole, sextupole, octupole, decapole and dodecapole correctors.

\subsection{The round coil superferric corrector}

In the initial phase of the design, the option of a superferric magnet based on $\mathrm{MgB}_{2}$ conductor was also considered. The major showstopper for using this technology in the superferric

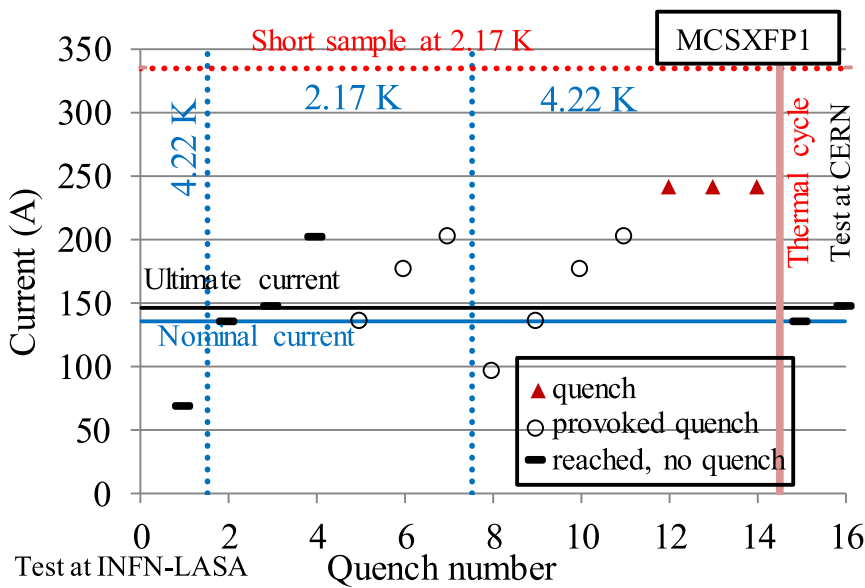

Figure 62. Training of sextupole corrector prototype.

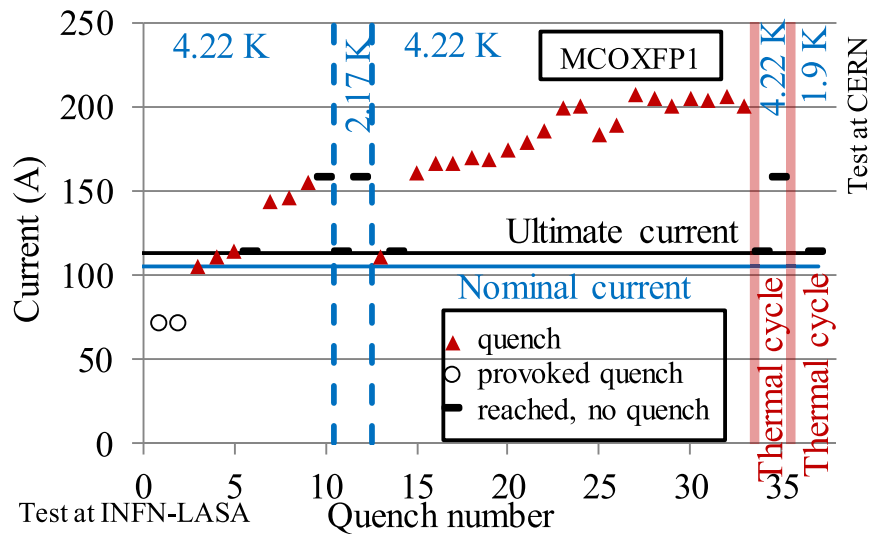

Figure 63. Training of octupole corrector prototype.

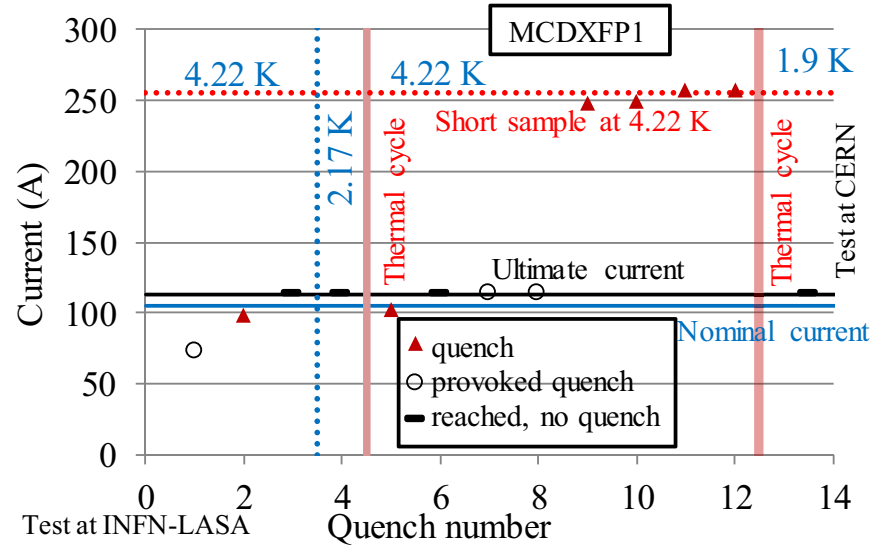

Figure 64. Training of decapole corrector prototype.

option was found to be the minimum curvature radius of the $\mathrm{MgB}_{2}$ tape, that had to be larger than $100 \mathrm{~mm}$. To avoid the small curvature radii, an alternative design based on a concept developed in the 70s [101] and further investigated in the 10s [102] has been explored [103]. The idea is to have a round coil whose solenoidal field is shaped in a multipolar transverse field thanks to the iron shape (round coil superconductor magnet, RCSM, see figure 68). 


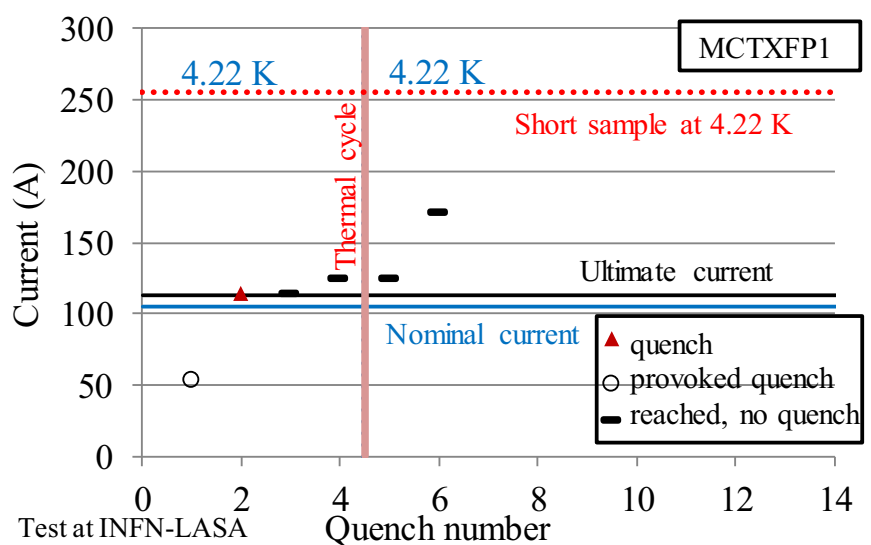

Figure 65. Training of dodecapole corrector prototype.

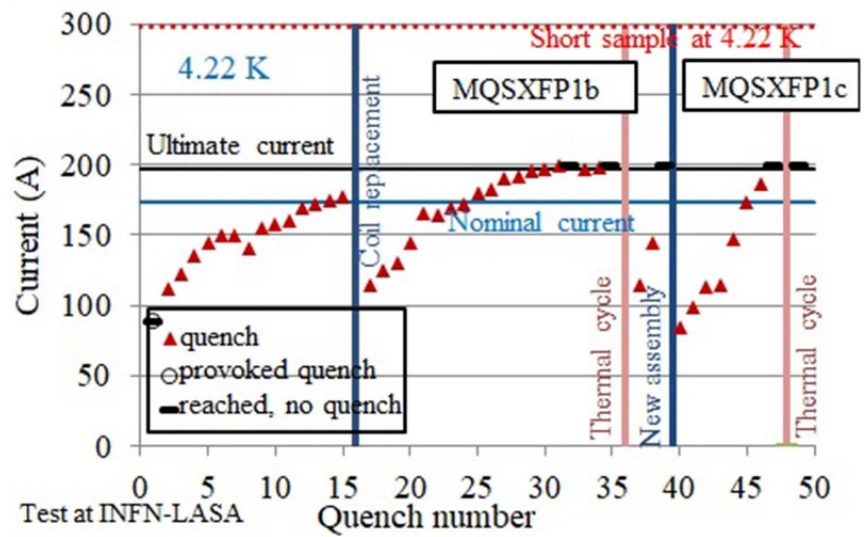

Figure 66. Training of skew quadrupole corrector prototype.

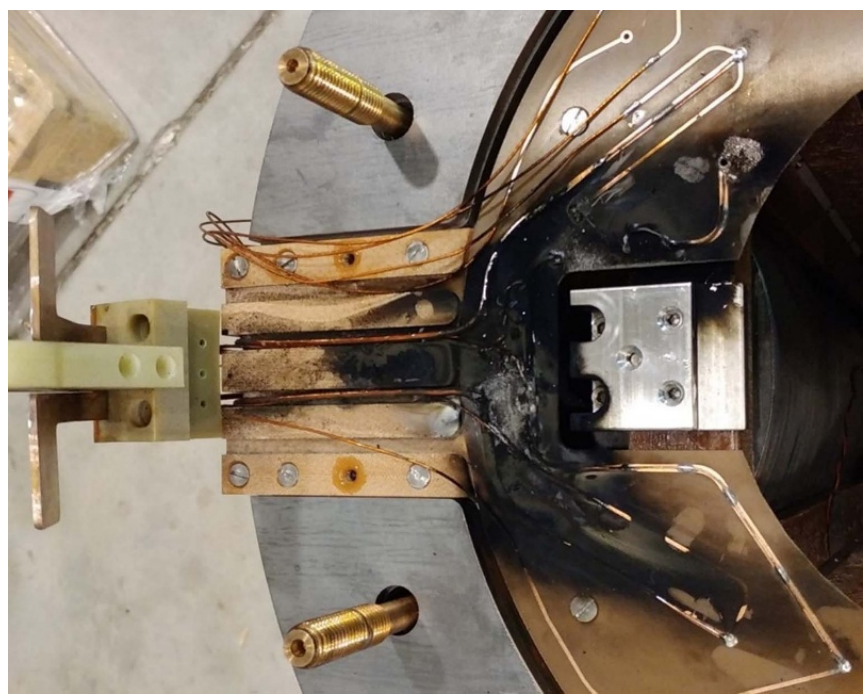

Figure 67. Side effects of the electrical short during test in the skew quadrupole.

This design has the main advantage of having not only much larger curvature radii for the coil, but also to use the same coil for producing different multipolar fields via the assembly in a different iron yoke. The drawback of this design

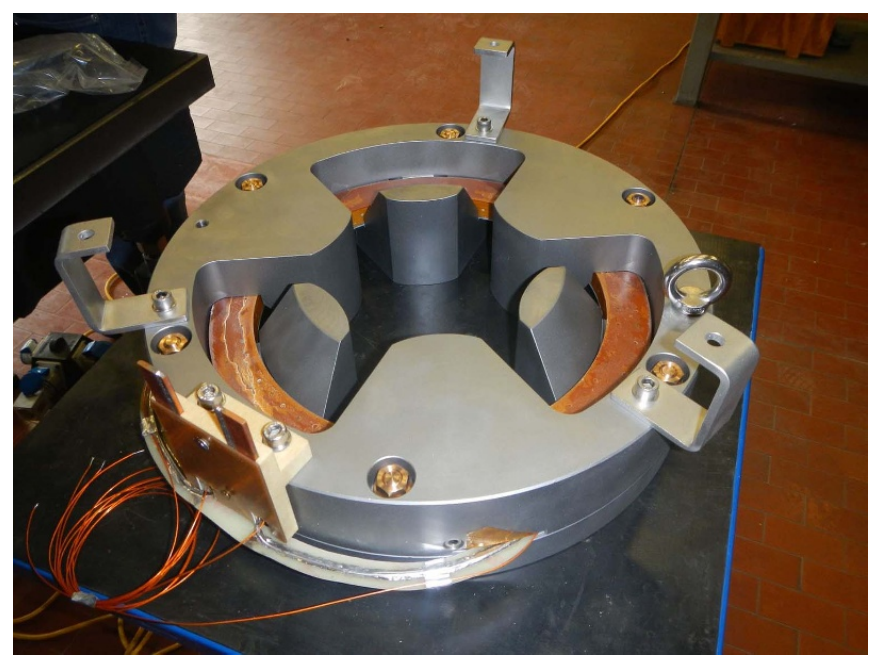

Figure 68. The final assembly of the sextupole corrector based on round coil superferric design and $\mathrm{MgB}_{2}$ conductor.

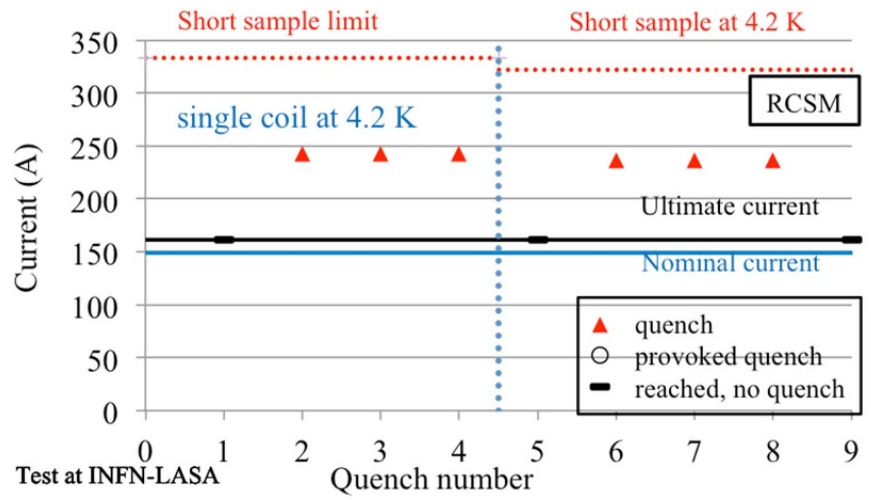

Figure 69. Training performance of sextupole corrector based on $\mathrm{RCSM}$ design and $\mathrm{MgB}_{2}$ conductor.

is compared to a standard superferric magnet one loses about a factor of two in the integrated gradient. Therefore, using this design would have required doubling the space for the corrector package, i.e. 3-4 additional meters: unfortunately this space was not available in the lay-out, and the option has been abandoned.

INFN pursed the construction of a sextupole demonstrator, i.e. half of a prototype, based on a $\mathrm{MgB}_{2}$ wire, able to carry $382 \mathrm{~A} \mathrm{~mm}^{-2}$ overall current density at $2.12 \mathrm{~T}$ and $4.2 \mathrm{~K}$. Nominal field is reached with a 150 A current, and the magnet operates at $45 \%$ of the loadline. The magnet test was carried out in LASA [104], and the magnet reached nominal current without quench, and was limited at $82 \%$ of the short sample field (see figure 69).

\subsection{Timeline}

The main milestones of the high order correctors development are the followings: 
- March 2014: signature of the collaboration agreement between CERN and INFN-LASA to develop design, manufacture and test five types of high order correctors;

- February 2016: test of the prototype sextupole;

- March 2017: test of the prototype octupole;

- August 2017: signature of the collaboration agreement between CERN and INFN-LASA to manufacture the high order corrector series;

- September 2017: test of the prototype decapole;

- September 2017: signature of contract with SAES-RIAL for manufacturing of dodecapole and quadrupole prototypes.

- April 2018: change of corrector strenght for quadrupole, sextupole, and octupole;

- October 2018: test of prototype dodecapole;

- February 2019: test of prototoype quadrupole, interrupted by an electrical short;

- July 2019: second test of the prototype quadrupole, after replacement of two coils.

Main bottleneck for the schedule is the coil winding and impregnation. This is particularly critical for magnets with higher number of poles. A production rate of one magnet every 2-3 weeks, depending on the magnet type, is assumed.

\section{Conclusions}

This paper describes the superconducting magnets needed for the HL-LHC IRs, that are now in transition between the model/prototype phase and the series production. It is a short series of about 150 magnets (including spares, prototypes and short models) of six different types: one quadrupole based on $\mathrm{Nb}_{3} \mathrm{Sn}$ technology, two main dipoles and three correctors based on $\mathrm{Nb}-\mathrm{Ti}$ technology.

The triplet represents a significant advancement in the accelerator magnets, with peak field 30\% larger than the $\mathrm{Nb}-\mathrm{Ti}$ LHC dipoles (11.4 $\mathrm{T}$ versus $8.7 \mathrm{~T}$ ) and twice accumulated midplane transverse stress in operational conditions (120 MPa versus $60 \mathrm{MPa}$ ).

The HL-LHC separation/recombination dipoles are in line with the existing $\mathrm{Nb}-\mathrm{Ti}$ technology, but present particular challenges:

- $\mathrm{D} 1$ is a $5.6 \mathrm{~T}$ magnet presenting a very large (100 MPa) accumulation of stress in the midplane due to its large aperture;

- D2 is a double aperture magnet where the coils are slightly asymmetric to compensate for the magnetic cross-talk, to reach the stringent target on field quality;

- The nested corrector has a peak field of $4.3 \mathrm{~T}$, but it relies on a double collared structure, that is a prima in accelerator magnets.

The triplet program was validated through the test of five short models and three prototypes, with a joint effort between CERN and a consortium of US laboratories (FNAL, BNL and LBNL). The magnets proved to be able to reach nominal current in four short models and two prototypes. Two production lines for short coils, and two production lines for $4.2 \mathrm{~m}$ long coil have been validated, showing to be able to manufacture coils reaching the required performance. The program had two understood failure cases, and two cases of totally or partially missing performance, most probabily related to conductor degradation, whose origin has not been clarified. The performance reproducibility is the main critical issue at the moment of writing. However, the MQXF program proved a large potential of $\mathrm{Nb}_{3} \mathrm{Sn}$ technology, in particular:

- Short models proved the existence of a wide range of assembly parameters (in terms of coil precompression) that provide the required performance;

- All magnets reaching nominal current at $1.9 \mathrm{~K}$ showed ability of reaching nominal current (and more) at $4.5 \mathrm{~K}$, thus proving a large temperature margin;

- All magnets showed no need of retraining to operate at nominal current after thermal cycle; in many cases no retraining was needed to operate even at ultimate current.

- A peak field of $13.4 \mathrm{~T}$ was reached, corresponding to $95 \%$ of short sample conditions at $1.9 \mathrm{~K}$, in one short model. This is $18 \%$ more than the field required for operating in the $\mathrm{LHC}$ at $7 \mathrm{TeV}$.

Finally, the MQXF magnet protection relies on CLIQ, i.e. a novel quench protection method based on heating the magnet via eddy currents generated by a capacitor discharge. This method has been sucesfully tested on the short models.

The separation dipole D1 design has been validated through the construction of three short models. The design and construction was done in KEK, Japan. Iterations have been required to optimize the precompression and field quality. At the end, all three models reached the target performance, the only missing point is the field quality fine tuning to minimize the sextupolar component.

The recombination dipole $\mathrm{D} 2$ design has been validated on two apertures. Design was done in INFN-Ge, Italy, and construction in ASG Superconductors. The strategy for the field quality cross-talk compensation based on an asymmetric coil geometry has been succesfully validated. The design required an iteration on the transition from the coil to the cable lead coming out from the winding pole. The initial design weakness provoked two shorts and one severely damaged cable.

The nested corrector proved the soundness of a double collared structure, a prima in magnet technology. Design and manufacturing was done in CIEMAT, Spain, with collaring at CERN. The double collaring allows to withstand the large torque when both magnets are powered. The prototype reached most of the required performance after few iterations on the azimuthal precompression of the straight part and of the heads.

The D2 orbit corrector using the canted $\cos \theta$ layout proved the flexibility and the advantages of this design requiring very little tooling, and simple and fast assembly. The magnet development was succesful and the technology was transfered to the collaboration led by IHEP, China.

The superferric correctors proved to be a very robust technology, and the design and prototype construction was done in INFN-LASA, Italy, with the longer magnets manufactured 
in SAES-RIAL. The validation has been completed with succesful tests, with few iterations on the design to improve the electrical insulation and the coil support.

The synergy between CERN and US laboratories allowed to develop the MQXF program along a short timeline, namely 32 months from aperture selection to short model magnet test, and 50 months from aperture selection to $4 \mathrm{~m}$ long prototype test. Typically, 3 years is the minimum time required from aperture selection to short model test for a known technology: this time has been 33 months for the Japanese program on D1, that profited of a preparatory work in the period 2011-2013. For the canted $\cos \theta$ corrector and for the high order correctors, 24 and 22 months were needed from the selection of the aperture to the test of the first prototype.

The project now enters the construction phase, the main challenge being the scaling from short model to prototypes for the main magnets. A difficult feature of the project is the small number of magnets to be built, allowing very limited feedback during production.

For the $\mathrm{Nb}_{3} \mathrm{Sn}$ case, that is the most innovative technology for superconducting magnets in accelerators, the HL-.LHC project will provide a statistics on the performance of 30 magnets and five prototypes built with identical cross-section and two different lengths, with three production lines. This will allow drawing precious conclusions for the potential of the $\mathrm{Nb}_{3} \mathrm{Sn}$ technology required in a high field collider to be built after the HL-LHC era.

\section{Acknowledgments}

This work was made possible thanks to the contribution of a large number of colleagues, much longer than the list of the authors. In particular we wish to acknowledge all the technicians involved in the prototyping and construction phases of all magnets and in all laboratories. We wish to thank B Strauss (DOE, retired) for his strong encouragement in pursuing the project, in all phases. Finally, a big thank to A Yamamoto (KEK) and D Tommasini (CERN) for serving as reviewers in several occasions, together with many others experts of the field.

\section{Appendix A. Stress estimate in sector magnets.}

In this section we give a more refined estimate for the accumulation of the stress in the midplane in a sector coil dipole and quadrupole. Following the approach outlined in [79], equation (3) for a dipole one can estimate the accumulation of stress in the midplane at the position $x$

$$
\sigma(x)=\frac{j^{2} \mu_{0} \sqrt{3}}{6 \pi(r+x)}\left[2(r+x)^{3}+r^{3}-3(r+x)^{2}(r+w)\right]
$$

where $x$ spans from 0 (at the magnet bore) to the coil width $w$ (on the outer radius of the coil). Since for a $60^{\circ}$ coil one has

$$
B=\gamma j w=\frac{\mu_{0} \sqrt{3}}{\pi} j w
$$

the previous equation can be cast in the form

$$
\sigma(x)=\frac{\operatorname{Bjr}}{2} \frac{2(r+x)^{3}+r^{3}-3(r+x)^{2}(r+w)}{3 w r(r+x)}
$$

and for $x=0$, i.e. on the edge of the aperture, one finds

$$
\sigma_{r}=\sigma(0)=\frac{B j r}{2} \frac{1}{3 w r^{2}}\left[-3 w r^{2}\right]=-\frac{B j r}{2} .
$$

Therefore the maximum of the stress is given by

$$
\sigma_{\max }=\sigma_{r} \operatorname{Max}_{0<x<w} \frac{2(r+x)^{3}+r^{3}-3(r+x)^{2}(r+w)}{3 w r(r+x)} .
$$

For a quadrupole one can compute (see equation (1) in [80], assuming a $30^{\circ}$ sector coil)

$$
\sigma(x)=\frac{j^{2} \mu_{0} \sqrt{3}}{16 \pi} \frac{(r+x)^{4}-r^{4}+4(r+x)^{4} \ln \left(\frac{r+w}{r+x}\right)}{(r+x)^{2}}
$$

where $x$ spans from 0 to the coil width $w$. For a 30 degrees coil one has

$$
G=\gamma j \ln \left(1+\frac{w}{r}\right)=\frac{\mu_{0} \sqrt{3}}{\pi} j \ln \left(1+\frac{w}{r}\right)
$$

the previous equation can be cast in the form

$$
\sigma(x)=\frac{j G}{16} \frac{(r+x)^{4}-r^{4}+4(r+x)^{4} \ln \left(\frac{r+w}{r+x}\right)}{(r+x)^{2} \ln \left(1+\frac{w}{r}\right)}
$$

and for $x=0$, i.e. on the edge of the aperture, one finds

$$
\sigma_{r}=\sigma(0)=\frac{j G}{4} r^{2}
$$

Therefore the maximum of the stress is given by

$$
\sigma_{\max }=\sigma_{r} \operatorname{Max}_{0<x<w} \frac{(r+x)^{4}-r^{4}+4(r+x)^{4} \ln \left(\frac{r+w}{r+x}\right)}{4 r^{2}(r+x)^{2} \ln \left(1+\frac{w}{r}\right)} .
$$

In table 6 we give the stress at the bore and the peak stress in the midplane for the HL-LHC magnets based on sector coils, for the $11 \mathrm{~T}$ and for the $\mathrm{LHC}$ dipole 
Table 6. Accumulated tranverse stress: at the bore (sr) and maximum inside the coil (smax) according to equations (A.5) and (A.10) for HL-LHC IR magnets.

\begin{tabular}{lllll}
\hline & $\begin{array}{l}w \\
(\mathrm{~mm})\end{array}$ & $\begin{array}{l}\sigma_{r} \\
(\mathrm{~mm})\end{array}$ & $\begin{array}{c}\sigma_{\max } / \sigma_{\mathrm{r}} \\
(\mathrm{MPa})\end{array}$ & $\begin{array}{l}\sigma_{\operatorname{mPa}} \\
(\mathrm{MPa})\end{array}$ \\
\hline $11 \mathrm{~T}$ & 30 & 28 & 90 & 116 \\
MQXF & 75 & 36 & 87 & 110 \\
LHC MB & 30 & 31 & 41 & 55 \\
MBXF & 75 & 15.4 & 94 & 99 \\
MBRD & 52.5 & 15.4 & 56 & 1.26 \\
MCBXF & 75 & 9 & 25 & 1.32 \\
\hline
\end{tabular}

\section{ORCID iDs}

E Todesco (iD https://orcid.org/0000-0001-5518-4191 S Izquierdo Bermudez (D) https://orcid.org/0000-0003-21574751

M Mentink (D) https://orcid.org/0000-0001-9769-0578

L Rossi (iD https://orcid.org/0000-0003-2158-7288

M Sugano (D) https://orcid.org/0000-0001-5168-3276

S Farinon (D) https://orcid.org/0000-0002-6487-2137

L Cooley (D) https://orcid.org/0000-0003-3488-2980

\section{References}

[1] Bruning O et al (eds) 2004 CERN-2004-003

[2] Perin R 1989 IEEE Trans. Magn. 25 1632-5

[3] Rossi L 2003 IEEE Trans. Appl. Supercond. 13 1221-8

[4] A A V V 1984 CERN 84-10

[5] The LHC study group 1995 CERN/AC/95-05

[6] Wyss C 1999 Particle Accelerator Conf. pp 149-53

[7] Billan J et al 1999 IEEE Trans. Appl. Supercond. 9 1039-44

[8] Lamont M et al 2009 Particle Accelerator Conf. pp 1412-4

[9] ATLAS Collaboration 2012 Phys. Lett. B 716 1-29

[10] CMS Collaboration 2012 Phys. Lett. B 716 30-61

[11] Rossi L 2012 J. Cryog. Supercond. Soc. Japan 47

[12] Strait J et al 2003 Particle Accelerator Conf. pp 42-4

[13] Bruning O et al 2002 LHC Project Report 626

[14] Koutchouk J P 2006 European Particle Accelerator Conf. p 556

[15] Bruning O and Rossi L (eds) 2015 Advanced Series on Directions in High Energy Physics vol 24 (Tokyo: World Scientific)

[16] Apollinari G et al (eds) 2017 CERN-2017-007-M

[17] Todesco E et al 2014 IEEE Trans. Appl. Supercond. 244003305

[18] Ajima Y et al 2005 Nucl. Instrum. Methods A 550 499-513

[19] Bossert R et al 2004 IEEE Trans. Appl. Supercond. 14 187-90

[20] Feher S et al 2006 IEEE Trans. Appl. Supercond. 16 437-40

[21] Willering G et al 2019 IEEE Trans. Appl. Supercond. 294004906

[22] Zlobin A V et al 2020 IEEE Trans. Appl. Supercond. 304001106

[23] Ferracin P et al 2005 IEEE Trans. Appl. Supercond. 15 1132-5

[24] Gourlay S et al 2006 IEEE Trans. Appl. Supercond. 16 324-7

[25] Wanderer P 2009 IEEE Trans. Appl. Supercond. 19 1208-11

[26] Caspi S 2010 IEEE Trans. Appl. Supercond. 20 144-7

[27] Ambrosio G et al 2008 IEEE Trans. Appl. Supercond. $13268-72$

[28] Ambrosio G et al 2013 IEEE Trans. Appl. Supercond. 234002204
[29] Ferracin P et al 2016 IEEE Trans. Appl. Supercond. 264000207

[30] Ferracin P et al 2019 IEEE Trans. Appl. Supercond. 294000207

[31] Todesco E 2018 IEEE Trans. Appl. Supercond. 284008809

[32] Scanlan R and Dietderich D R 2003 IEEE Trans. Appl. Supercond. 13 1536-41

[33] Asner A et al 1989 Proc. of MT-11 (New York: Elsevier Applied Science) pp. 36-41

[34] Ten Kate H J, Den Ouden A, Ter Avest D, Wessel S, Dubbeldam R, Van Emden W, Daum C, Bona M and Perin R 1991 IEEE Trans. Magn. 27 1996-9

[35] Benjegerdes R et al 1999 Particle Accelerator Conf. 3233-5 (D20)

[36] Sabbi G et al 2005 IEEE Trans. Appl. Supercond. 15 1128-31

[37] Lietzke A et al 2004 IEEE Trans. Appl. Supercond. $14345-8$

[38] Izquiredo Bermudez S et al 2017 IEEE Trans. Appl. Supercond. 174002004

[39] Weijers H W, Ten Kate H and Van Oort J M 1993 IEEE Trans. Appl. Supercond. 31334

[40] Ten Haken B, Godeke A and Ten Kate H H J 1999 J. Appl. Phys. 85 3247-53

[41] Savary F 2017 IEEE Trans. Appl. Supercond. 274003505

[42] Tommasini D et al 2017 IEEE Trans. Appl. Supercond. 274000405

[43] Tommasini D et al 2018 IEEE Trans. Appl. Supercond. 284001305

[44] Xu Q, Nakamoto T, Ogitsu T, Sasaki K, Terashima A, Tsuchiya K, Yamamoto A, Todesco E, Kikuchi A and Takeuchi T 2012 IEEE Trans. Appl. Supercond. 224901404

[45] Nakamoto T et al 2015 IEEE Trans. Appl. Supercond. 254000505

[46] Sugano M et al 2017 IEEE Trans. Appl. Supercond. 274002409

[47] Sugano M et al 2018 IEEE Trans. Appl. Supercond. 284000805

[48] Enomoto S et al 2018 IEEE Trans. Appl. Supercond. 284004505

[49] Nilsson E, Bermudez S I, Todesco E, Enomoto S, Farinon S, Fabbricatore P, Nakamoto T, Sugano M and Savary F 2018 IEEE Trans. Appl. Supercond. 284003005

[50] Suzuki K et al 2019 IEEE Trans. Appl. Supercond. 294000905

[51] Sugano M et al 2019 IEEE Trans. Appl. Supercond. 294003607

[52] Farinon S, Fabbricatore P, Curreli S and Todesco E 2016 IEEE Trans. Appl. Supercond. 264001504

[53] Fabbricatore P, Bersani A, Caiffi B, Cereseto R, Farinon S, Foussat A and Todesco E 2018 IEEE Trans. Appl. Supercond. 284000105

[54] Bersani A, Caiffi B, Cereseto R, Fabbricatore P, Farinon S, Foussat A P, Bahmane O I, Levi F, Sahner T and Todesco E 2019 IEEE Trans. Appl. Supercond. 294003305 
[55] Foussat A et al 2020 IEEE Trans. Appl. Supercond. 304003405

[56] Kashikin V and Zlobin A 2007 Particle Accelrator Conf. vol 464-466

[57] Ogitsu T et al 2005 IEEE Trans. Appl. Supercond. $151175-80$

[58] Garcia-Matos J, Toral F and Fessia P 2016 IEEE Trans. Appl. Supercond. 264102005

[59] Garcia-Matos J et al 2018 IEEE Trans. Appl. Supercond. 284101005

[60] Garcia-Matos J et al 2019 IEEE Trans. Appl. Supercond. 294002205

[61] Kirby G et al 2017 IEEE Trans. Appl. Supercond. 274002805

[62] Kirby G et al 2018 IEEE Trans. Appl. Supercond. 284002205

[63] Mangiarotti F et al 2019 IEEE Trans. Appl. Supercond. 29 4002305

[64] Meyer D I and Flasck R 1970 Nucl. Instrum. Methods $80339-41$

[65] Goodzeit C L, Ball M J and Meinke R B 2003 IEEE Trans. Appl. Supercond. 13 1365-8

[66] Caspi S et al 2014 IEEE Trans. Appl. Supercond. 244001804

[67] Volpini G et al 2015 IEEE Trans. Appl. Supercond. 254002605

[68] Statera M et al 2017 IEEE Trans. Appl. Supercond. 274003205

[69] Statera M et al 2018 IEEE Trans. Appl. Supercond. 284008705

[70] Sorbi M et al 2018 IEEE Trans. Appl. Supercond. 284100205

[71] Statera M et al 2019 IEEE Trans. Appl. Supercond. 294004305

[72] Sorbi M et al 2019 IEEE Trans. Appl. Supercond. 294001905

[73] Fiscarelli L, Bajas H, Mangiarotti F, Musso A, Russenchuck S, Mariotto S, Sorbi M and Statera M 2019 IEEE Trans. Appl. Supercond. 294003505

[74] Abramian P, Calero J, Garcia-Tabares L, Molina E, Rodriguez I and Toral F 2012 IEEE Trans. Appl. Supercond. 224902104

[75] Abramian P et al 2013 IEEE Trans. Appl. Supercond. 234101204

[76] Tollestrup A and Todesco E 2008 Rev. Accel Sci. Technol. 1 185-210

[77] Bordini B, Barzi E, Feher S, Rossi L and Zlobin A V 2008 IEEE Trans. Appl. Supercond. 18 1309-12

[78] Bordini B, Bajko M, Caspi S, Dietderich D, Felice H, Ferracin P, Rossi L, Sabbi G L and Takala E 2010 IEEE Trans. Appl. Supercond. 20 274-8

[79] Ravaioli E et al 2015 IEEE Trans. Appl. Supercond. 224001305
[80] Felice H et al 2018 IEEE Trans. Appl. Supercond. 28 4500105

[81] Rossi L and Todesco E 2007 Phys. Rev. Spec. Top. Accel Beams 10112401

[82] Rossi L and Todesco E 2006 Phys. Rev. Spec. Top. Accel Beams 9102401

[83] Fessia P, Regis F and Todesco E 2009 IEEE Trans. Appl. Supercond. 19 1203-7

[84] Fessia P, Regis F and Todesco E 2007 IEEE Trans. Appl. Supercond. 17 1269-72

[85] Todesco E 2013 CERN 2013-006 10-16

[86] Cooley L, Ghosh A K, Dietderich D R and Pong I 2017 IEEE Trans. Appl. Supercond. 276000505

[87] Bordini B, Ballarino A, Macchini M, Richter D, Sailer B, Thoener M and Schlenga K 2017 IEEE Trans. Appl. Supercond. 276000706

[88] Izquiredo Bermudez S et al 2018 IEEE Trans. Appl. Supercond. 284008406

[89] Levitan J, Lu J, Lombardo V and Cooley L 2019 IEEE Trans. Appl. Supercond. 296000904

[90] Pong I, Cooley L D, Lin A, Higley H C and Sanabria C 2019 IEEE Trans. Appl. Supercond. 296001505

[91] Chlachidze G et al 2017 IEEE Trans. Appl. Supercond. 274000205

[92] Stoynev S et al 2018 IEEE Trans. Appl. Supercond. 284001705

[93] Bajas H et al 2018 IEEE Trans. Appl. Supercond. 284007006

[94] Mangiarotti F et al 2019 IEEE Trans. Appl. Supercond. 294001705

[95] Muratore J et al 2020 IEEE Trans. Appl. Supercond. 30

[96] Izquierdo Bermudez S et al 2019 IEEE Trans. Appl. Supercond. 294901705

[97] Ravaioli E et al 2019 IEEE Trans. Appl. Supercond. 294701405

[98] Fleiter J et al 2017 IEEE Trans. Appl. Supercond. 274004305

[99] Anarella M et al 2003 Nucl. Instrum. Methods A 499 280-315

[100] Sorbi M, Alessandria F, Bellomo G, Fabbricatore P, Farinon S, Gambardella U and Volpini G 2008 IEEE Trans. Appl. Supercond. 18 138-41

[101] Malyshev I F 1973 U. S. Patent 1698 890/26-25

[102] Kashikhin V 2010 IEEE Trans. Appl. Supercond. 20 196-200

[103] Mariotto S, Marinozzi V, Rysti J, Sorbi M and Statera M 2018 IEEE Trans. Appl. Supercond. 284003305

[104] Mariotto S, Leone A, Paccalini A, Pasini A, Pedrini D, Quadrio M, Sorbi M, Statera M, Todero M and Valente R 2019 IEEE Trans. Appl. Supercond. 294004505 